\title{
An annotated checklist of chewing lice (Phthiraptera: Amblycera, Ischnocera) from Slovakia
}

LUCIE OŠLEJŠKOVÁ ${ }^{1,4}$, JÁN KRIŠTOFÍK ${ }^{2}$, ALFRÉD TRNKA ${ }^{3}$ \& OLDŘICH SYCHRA ${ }^{1 *}$

${ }^{1}$ Department of Biology and Wildife Diseases, Faculty of Veterinary Hygiene and Ecology, University of Veterinary Sciences Brno, Palackého tř. 1946/1, 61242 Brno, Czech Republic.

${ }^{2}$ Institute of Zoology, Slovak Academy of Sciences, Dúbravsk cesta 9, 84506 Bratislava, Slovakia.

”=jan.kristofik@savba.sk; @ https://orcid.org/0000-0002-7149-1164

${ }^{3}$ Department of Biology, University of Trnava, Priemyselna 4, 91843 Trnava, Slovakia.

"=alfred.trnka@truni.sk; @ https://orcid.org/0000-0002-2609-678X

4 "'oslejskoval@vfu.cz; @ ittps://orcid.org/0000-0002-1667-6748

*Corresponding author. !"sychrao@vfu.cz; ๑ https://orcid.org/0000-0003-3481-5673

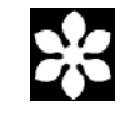

Magnolia Press

Auckland, New Zealand 
LUCIE OŠLEJŠKOVÁ, JÁN KRIŠTOFÍK, ALFRÉD TRNKA \& OLDŘICH SYCHRA An annotated checklist of chewing lice (Phthiraptera: Amblycera, Ischnocera) from Slovakia (Zootaxa 5069)

80 pp.; $30 \mathrm{~cm}$.

19 Nov. 2021

ISBN 978-1-77688-412-4 (paperback)

ISBN 978-1-77688-413-1 (Online edition)

FIRST PUBLISHED IN 2021 BY

Magnolia Press

P.O. Box 41-383

Auckland 1041

New Zealand

e-mail: magnolia@mapress.com

https://www.mapress.com/j/zt

(C) 2021 Magnolia Press

ISSN 1175-5326 (Print edition)

ISSN 1175-5334 (Online edition) 


\section{Table of Contents}

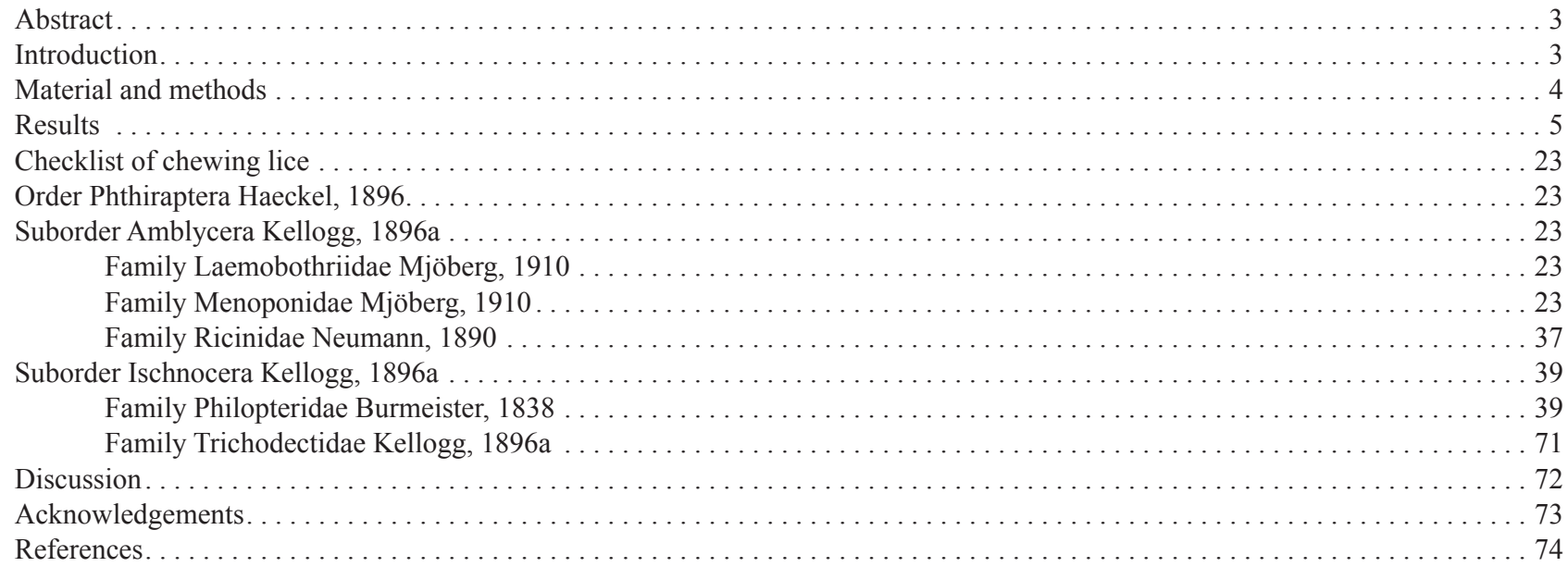

\section{Abstract}

This checklist includes taxa of chewing lice from published records, old collections, and recently collected material from birds and mammals in Slovakia. Data from established collections correspond to five different periods: (1) 1925-1939, collection of Karel Pfleger; (2) 1946-1978, collection of František Balát; (3) 1974-1985, collection of Vladimír Straka; (4) 1997-2012, collection of Ján Krištofík; and (5) 2008-2019, a collection made by the authors of this paper. A total of 255 species of feather lice - 67 amblyceran species in 22 genera of families Laemobothriidae, Menoponidae and Ricinidae, and 188 ischnoceran species in 54 genera of the family Philopteridae - and 366 host-louse associations are listed from 171 bird species in 21 orders. In addition, eight species of chewing lice in five genera of the family Trichodectidae are listed from eight species of mammals. Species of chewing lice are reported from about 240 different locations throughout the territory of Slovakia. Also, 43 species of lice and 20 host-louse associations for Slovakia, as well as four host-louse associations for the world, are included as new records. A host-louse list of recorded species is also given.

Key words: Phthiraptera, Amblycera, Ischnocera, chewing lice, birds, mammals, checklist, species, host-louse associations, new records, Slovakia

\section{Introduction}

Chewing lice are common ectoparasites of birds and some groups of mammals. Together with sucking lice, they comprise the insect order Phthiraptera, which is divided in four suborders: Amblycera, Ischnocera (comprising lice from birds and mammals), Rhynchopthirina and Anoplura (comprising lice from mammals only) (Price et al. 2003; Durden \& Musser 1994). Due to the obligate life cycle of lice in close association with their hosts, strong host specificity is traditionally expected, hence lice are very often used as models for cophylogenetic studies (Clayton et al. 2016). However, recent studies at lower taxonomic levels have revealed that multi-host louse species may be more common than expected, and that one genus of lice may contain species ranging from monoxenous to polyxenous in their host distributions (Martinů et al. 2015; Sychra et al. 2021).

Birds usually carry one amblyceran species and one ischnoceran species, but many hosts harbour more than two species (Price et al. 2003). Although the impact of chewing lice on wild animals is difficult to measure, there are reports of single hosts carrying populations numbering in the hundreds or more without any obvious negative effect on the host (Naz et al. 2010; Ošlejšková et al. 2020). However, it has been documented that some groups of lice can affect important host functions negatively, such as flight performance (Barbosa et al. 2002), metabolic rate (Booth et al. 1993), sexual selection (Kose \& Mller 1999), grooming time (Cotgreave \& Clayton 1994), reproductive success (Tompkins et al. 1996), migration (Literák et al. 2015), and even host survival (Brown et al. 1995). The relative host specificity of lice is important from a conservation viewpoint, as endangered and extinct hosts also carry endangered and extinct lice. Rózsa \& Vas (2015) reported louse species which are extinct or endangered together with their hosts. 
Therefore, to evaluate the impact of chewing lice on different populations of their hosts throughout their area of distribution, we first need to know the diversity of louse species and their host associations. It is well documented that some groups of lice do not occur over the entire distribution of their hosts (Clay 1949a; Bush et al. 2009). Hence, it is also necessary to know the geographic distribution of louse species as precisely as possible, beginning with accurate regional checklists as sources of information.

A number of checklists of chewing lice from Slovakia and neighboring countries are available, as follows: Czechoslovakia (Balát 1977), Poland (Złotorzycka 1972a,b, 1976, 1977, 1978, 1980) and Ukraine (Fedorenko 1983, 1987). In addition, there are more recent checklists from other parts of central and eastern Europe, e.g. Germany (Mey 2003), Bulgaria (Ilieva 2009), Hungary (Vaz et al. 2012a,b) and Romania (Adam 2008).

In his checklist of chewing lice from former Czechoslovakia, Balát (1977) reported 177 species of which 169 are still valid according to the taxonomy of Price et al. (2003). However, Balát (1977) gives no information about host associations. Overviews of host-louse associations were published by Balát (1955a, 1956), who recorded 156 species of lice from 127 hosts with a total of 211 host-louse associations from 75 locations. Subsequently, Straka (1987) recorded 105 species of lice from 81 hosts with a total of 111 host-louse associations from an additional 33 locations. Other partial reports of lice from Slovakia were published by Balát (1952, 1981a), Štefan (1977), Straka (1982), Mey (1988), Máca (1991), Lukáš et al. (1991, 1992), Krištofík et al. (1996), Krištofík (1999, 2000), Janiga \& Kubašková (2000), Janiga \& Mičková (2004), Goldová et al. (2006), Szczykutowicz et al. (2006), Sychra et al. (2008), Martinů et al. (2015), Bush et al. (2018), Janiga (2018, 2019) and Najer et al. (2020).

From the foregoing, it is clear that there has not been a comprehensive account of all the chewing lice recorded from Slovakia, including hosts and locations. Therefore, the aim of this paper is to list all available records of chewing lice, published and new, from the territory of Slovakia and provide annotations regarding their host-louse associations. Also, a host-louse list is given in Table 5.

\title{
Material and methods
}

We reviewed all available publications reporting data about chewing lice from Slovakia and examined collections held in the following institutions:

University of Veterinary Sciences, Brno, Czech Republic (VETUNI)

Moravian Museum, Brno, Czech Republic (MMBC)

National Museum, Prague, Czech Republic (NMPC)

Slovak National Museum, Bratislava, Slovakia (SNMB)

Slovak National Museum, (Andrej Kmet' Museum), Martin, Slovakia (AKMM)

Natural History Museum, London, England (NHML)

Each louse species entry contains the following information:

\author{
Genus species author/s, date of publication \\ Host: \\ Reference/s: \\ Location/s: \\ Note/s:
}

A louse species recorded from more than one host has as many entries as number of hosts. Most references are to papers published by Balát (1953, 1955a, 1956, 1977) and Straka (1987) who provided detailed checklists of lice, including data about hosts, locations and dates of collections. In addition, Balát (1953) provided a list of lice and their hosts from Moravia and Slovakia, but without specifying the country for each host-louse association. Balát (1977) published a checklist of chewing lice from the territory of former Czechoslovakia, noting whether the species was found in Bohemia, Moravia or Slovakia, but there are no records of hosts in this paper. Host-louse associations, but without locations, were summarised by Balát in sections Parasites for particular birds in Hudec \& Černý (1972, 1977) and Hudec (1983). Similarly, Krištofík summarised host-louse associations in section Parasites for mammals 
in Krištofík \& Danko (2012). Many other papers dealing with small numbers of louse species are also cited in the references section.

The section "Location/s mainly refers to data from the František Balát Collection of permanent slides (Balát Coll.) deposited in the MMBC and the SNMB. These collections comprise about 300 slides with specimens collected during 1946-1978 from about 85 locations. Where possible, we recorded information from Balát's original handwritten list of slides, which includes data about (1) louse taxa, with numbers of males, females and/or nymphs collected; (2) host taxa, their sex and/or age; (3) locality: state, detailed location and date; (4) various notes. Almost all the notes correspond with those written on slides and/or in Balát's field diaries from 1953-1963, when available. Unless otherwise stated, all data up to 1956 were published by Balát (1956). Besides the locations taken from slide labels, some locations were taken from Balát's publications (Balát 1952, 1955, 1956). We record slide numbers, although not all of the slides are present in the Balát Collection. Also, we corrected obvious misspellings of location names and dates, taking data from the notes of Balát Collection as correct.

In addition to published records, we identified all specimens in the slides of the Balát Collection and, with only a few exceptions, all lice were correctly determined; therefore, we accepted data from Balát's list about missing slides as correct. In some cases, where the slides and/or specimens were in poor condition, we accepted Balát's identification, especially if the host-louse association agreed with other known records. We are aware that the practice of identifying lice solely on their host association is not appropriate. Therefore, in some cases, we left the identification at the genus level only; most of these lice belong to the genus Philopterus, which is in need of a comprehensive systematic revision.

We have included data from the collection of lice made by Karel Pfleger (Pfleger Coll.) deposited in the NMPC, the MMBC and the SNMB. In total, this collection comprises about 320 slides with lice collected during 1925-1939 from about 12 locations. Another collection of lice included in this list, and collected by Vladimír Straka during 1974-1985, comprises about 2100 specimens on almost 500 slides deposited at the Slovak National Museum in Martin (Andrej Kmet Museum), Slovakia (Straka Coll.; see Straka 1987). Finally, we included a collection of lice from Lanius excubitor collected by Tibor Weisz (Weisz Coll.) during 1963-1964 (see Szczykutowicz et al. 2006).

More recent collections of lice from Slovakia are: (1) the Ján Krištofík Collection (Krištofík Coll.) made during 1997-2012 from about 52 different locations, and (2) a collection made by L. Ošlejšková, A. Trnka and O. Sychra in Gbelce (see Sychra et al. 2008; Martinů et al. 2015; Najer et al. 2020). All lice, slide-mounted and in alcohol, from these two collections are deposited in the VETUNI, unless otherwise stated, and their data are published for the first time in this paper.

In the Checklist, all taxa are listed in alphabetical order: families within each suborder, genera within each family, and species within each genus. Taxonomy and nomenclature of chewing lice follow Price et al. (2003), with updates from (1) Mey (2004) regarding the genera of the Philopterus-complex, (2) Grossi et al. (2014) on the Anatoecus dentatus-complex, and (3) Gustafsson \& Bush (2017) regarding the Brueelia-complex. The taxonomy and nomenclature of birds follow IOC Bird World List ver. 10.1 (Gill et al. 2021), and we used the list of birds of Slovakia produced by the Slovak Ornithological Society (SOS/BirdLife Slovakia 2020), which includes 359 native species (see Table S1). We added two widespread feral species introduced by humans-Phasianus colchicus and Columba livia - and five species of captive birds - Gallus gallus, Numida meleagris, Pavo cristatus, Chrysolophus pictus and Bubo scandiacus - which have been found with lice. Taxonomy and nomenclature of mammals follow the list by Krištofík \& Danko (2012).

\section{Results}

A total of 263 species of chewing lice from Slovakia -67 amblyceran species in 22 genera and 196 ischnoceran species in 59 genera from 171 species of birds in 21 orders, and eight species of mammals in two orders - are listed in this paper. Also, a total of 366 host-louse associations (347 as species plus 19 as genus only) are recorded (Tables 1-5). These records are from about 240 different locations throughout the territory of Slovakia.

The total of 263 species of chewing lice and 347 host-louse associations at the species level includes 43 species and 20 host-louse associations recorded for the first time from Slovakia (Tables 4, 5). In addition, we record three new host-louse associations for the world, as follows: Holomenopon clypeilargum from Mergellus albellus, Kurodaia (Conciella) cryptostigmatia from Strix uralensis, Menacanthus eurysternus from Turdus iliacus and Myrsidea 
thoracica from Turdus torquatus (Table 4). Average numbers of host species per louse for different genera of lice are given in Table 4.

The proportion of native host species with at least one species of louse recorded from them is very high (95.5\%), with only nine introduced and captive hosts (Table 1). At least two species of chewing lice, one from each suborder-Amblycera and Ischnocera - were recorded from 77 (42\%) species of birds from a total of 182 host species. A slightly higher proportion of hosts (49\%) was parasitised by ischnoceran species only, while $16(9 \%)$ host species harboured members of Amblycera only.

TABLE 1. Numbers of host species and host-louse associations (including genus level) from birds and mammals recorded in Slovakia.

\begin{tabular}{llll}
\hline & Number of host species & Host-louse associations & Number of louse species per host \\
\hline BIRDS & $\mathbf{1 7 1}$ & $\mathbf{3 5 8}$ & $\mathbf{2 . 1}$ \\
Native & 164 & 338 & 2.1 \\
Introduced & 2 & 9 & 4.5 \\
Captive & 5 & 11 & 2.2 \\
& & & \\
MAMMALS & $\mathbf{8}$ & $\mathbf{8}$ & $\mathbf{1 . 0}$ \\
Native & 6 & 6 & 1.0 \\
Introduced & 0 & 0 & - \\
Captive & 2 & 2 & 1.0 \\
TOTAL & $\mathbf{1 7 9}$ & $\mathbf{3 6 6}$ & $\mathbf{2 . 0}$ \\
\hline
\end{tabular}

TABLE 2. Suborders and families of chewing lice with numbers of louse genera, species and host-louse associations (excluding records at genus level) recorded in Slovakia.

\begin{tabular}{lllll}
\hline & $\begin{array}{l}\text { Number of louse } \\
\text { genera }\end{array}$ & $\begin{array}{l}\text { Number of louse } \\
\text { species }\end{array}$ & Host-louse associations & $\begin{array}{l}\text { Number of host } \\
\text { species per louse }\end{array}$ \\
\hline Amblycera & $\mathbf{2 2}$ & $\mathbf{6 7}$ & $\mathbf{1 0 8}$ & $\mathbf{1 . 6}$ \\
Laemobothriidae & 1 & 2 & 2 & 1.0 \\
Menoponidae & 20 & 60 & 95 & 1.5 \\
Ricinidae & 1 & 5 & 11 & 2.2 \\
Ischnocera & $\mathbf{5 9}$ & $\mathbf{1 9 6}$ & $\mathbf{2 3 9}$ & $\mathbf{1 . 3}$ \\
Philopteridae & 54 & 188 & $\mathbf{2 3 1}$ & 1.3 \\
Trichodectidae & 5 & 8 & 8 & 1.0 \\
TOTAL & $\mathbf{8 1}$ & $\mathbf{2 6 3}$ & $\mathbf{3 4 7}$ & $\mathbf{1 . 3}$ \\
\hline
\end{tabular}

Average numbers of louse species per host, for orders and families of hosts, are given in Table 3. With seven species of lice, Phasianus colchicus has the highest louse diversity, followed by Corvus frugilegus with six, and Coloeus monedula, Passer montanus and Turdus pilaris with five each (Table 5). The bird orders Bucerotiformes, Caprimulgiformes, Ciconiiformes, Coraciiformes, Galliformes and Piciformes had a higher proportion of host species $(80-100 \%)$ with recorded lice in comparison to host-louse associations recorded from the world. The lowest proportion $(20 \%)$ was in the Charadriiformes.

Considering the host birds recorded in Slovakia and what lice are known from them elsewhere, these louse genera have the highest proportions of recorded louse species in this country: Alcedoffula, Cincloecus, Cuculoecus, Damalinia, Eidmanniella, Ibidoecus, Gruimenopon, Maculinirmus, Meropoecus, Meropsiella, Mulcticola, Neophilopterus, Turdinirmus, Trinoton and Upupicola. The lowest proportions (7-14\%) are for Actornithophilus, Austromenopon, Carduiceps, Lunaceps and Saemundssonia (Table 4). The highest host ranges for individual species are for Anatoecus dentatus (eight host species), Menacanthus eurysternus (eight) and Trinoton querquedulae (seven) (Table 5). 
TABLE 3. List of host taxa with numbers of species and host-louse associations (including records at genus level) recorded in Slovakia.

\begin{tabular}{|c|c|c|c|c|}
\hline & $\begin{array}{l}\text { Number of host } \\
\text { species }\end{array}$ & Host-louse associations & $\begin{array}{l}\text { Number of louse species per } \\
\text { host }\end{array}$ & Range \\
\hline NATIVE BIRDS & 164 & 338 & 2.1 & $1-6$ \\
\hline Accipitriformes & 16 & 33 & 2.1 & $1-4$ \\
\hline Accipitridae & 15 & 32 & 2.1 & $1-4$ \\
\hline Pandionidae & 1 & 1 & 1.0 & \\
\hline Anseriformes & 15 & 32 & 2.1 & $1-4$ \\
\hline Anatidae & 15 & 32 & 2.1 & $1-4$ \\
\hline Apodiformes & 1 & 1 & 1.0 & \\
\hline Apodidae & 1 & 1 & 1.0 & \\
\hline Bucerotiformes & 1 & 2 & 2.0 & \\
\hline Upupidae & 1 & 2 & 2.0 & \\
\hline Caprimulgiformes & 1 & 1 & 1.0 & \\
\hline Caprimulgidae & 1 & 1 & 1.0 & \\
\hline Charadriiformes & 14 & 25 & 1.8 & 1-3 \\
\hline Charadriidae & 3 & 5 & 1.6 & $1-2$ \\
\hline Laridae & 5 & 9 & 1.8 & $1-3$ \\
\hline Scolopacidae & 6 & 11 & 1.8 & $1-3$ \\
\hline Ciconiiformes & 2 & 4 & 2.0 & 1-3 \\
\hline Ciconiidae & 2 & 4 & 2.0 & $1-3$ \\
\hline Columbiformes & 3 & 6 & 2.0 & 1-3 \\
\hline Columbidae & 3 & 6 & 2.0 & $1-3$ \\
\hline Coraciiformes & 3 & 5 & 1.7 & 1-3 \\
\hline Alcedinidae & 1 & 1 & 1.0 & \\
\hline Coraciidae & 1 & 1 & 2.0 & \\
\hline Meropidae & 1 & 3 & 3.0 & \\
\hline Cuculiformes & 1 & 2 & 2.0 & \\
\hline Cuculidae & 1 & 2 & 2.0 & \\
\hline Falconiformes & 3 & 7 & 2.3 & $1-4$ \\
\hline Falconidae & 3 & 7 & 2.3 & $1-4$ \\
\hline Galliformes & 4 & 10 & 2.5 & 1-3 \\
\hline Phasianidae & 4 & 10 & 2.5 & $1-3$ \\
\hline Gaviiformes & 1 & 1 & 1.0 & \\
\hline Gaviidae & 1 & 1 & 1.0 & \\
\hline Gruiformes & 4 & 14 & 3.5 & $1-4$ \\
\hline Gruidae & 1 & 4 & 4.0 & \\
\hline Rallidae & 3 & 10 & 3.3 & $1-4$ \\
\hline Otidiformes & 1 & 1 & 1.0 & \\
\hline Otididae & 1 & 1 & 1.0 & \\
\hline Passeriformes & 69 & 154 & 2.2 & $1-6$ \\
\hline Acrocephalidae & 4 & 8 & 2.0 & $1-2$ \\
\hline Aegithalidae & 1 & 1 & 1.0 & \\
\hline Alaudidae & 2 & 5 & 2.5 & $2-3$ \\
\hline
\end{tabular}




\begin{tabular}{|c|c|c|c|c|}
\hline & $\begin{array}{l}\text { Number of host } \\
\text { species }\end{array}$ & Host-louse associations & $\begin{array}{l}\text { Number of louse species per } \\
\text { host }\end{array}$ & Range \\
\hline Certhiidae & 1 & 1 & 1.0 & \\
\hline Cinclidae & 1 & 2 & 2.0 & \\
\hline Corvidae & 7 & 24 & 3.4 & $1-6$ \\
\hline Emberizidae & 2 & 7 & 3.5 & $3-4$ \\
\hline Fringillidae & 10 & 16 & 1.6 & $1-3$ \\
\hline Hirundinidae & 3 & 8 & 2.7 & $2-3$ \\
\hline Laniidae & 3 & 8 & 2.7 & $1-3$ \\
\hline Locustellidae & 2 & 3 & 1.5 & $1-2$ \\
\hline Motacillidae & 5 & 9 & 1.8 & $1-3$ \\
\hline Muscicapidae & 4 & 6 & 1.5 & $1-2$ \\
\hline Oriolidae & 1 & 3 & 3.0 & \\
\hline Panuridae & 1 & 2 & 2.0 & \\
\hline Paridae & 4 & 8 & 2.3 & $1-4$ \\
\hline Passeridae & 2 & 7 & 3.5 & $2-5$ \\
\hline Phylloscopidae & 2 & 2 & 1.0 & \\
\hline Prunellidae & 2 & 3 & 1.5 & $1-2$ \\
\hline Regulidae & 1 & 1 & 1.0 & \\
\hline Remizidae & 1 & 2 & 2.0 & \\
\hline Sittidae & 1 & 2 & 2.0 & \\
\hline Sturnidae & 1 & 4 & 4.0 & \\
\hline Sylviidae & 1 & 1 & 1.0 & \\
\hline Troglodytidae & 1 & 2 & 2.0 & \\
\hline Turdidae & 6 & 19 & 3.2 & $1-5$ \\
\hline Pelecaniformes & 6 & 8 & 1.3 & $1-2$ \\
\hline Ardeidae & 4 & 5 & 1.3 & $1-2$ \\
\hline Threskiornithidae & 2 & 3 & 1.5 & $1-2$ \\
\hline Piciformes & 8 & 16 & 2.0 & 1-3 \\
\hline Picidae & 8 & 16 & 2.0 & $1-3$ \\
\hline Podicipediformes & 3 & 4 & 1.3 & $1-2$ \\
\hline Podicipedidae & 3 & 4 & 1.3 & $1-2$ \\
\hline Strigiformes & 7 & 10 & 1.4 & 1-2 \\
\hline Strigidae & 6 & 8 & 1.3 & $1-2$ \\
\hline Tytonidae & 1 & 2 & 2.0 & \\
\hline Suliformes & 1 & 2 & 2.0 & \\
\hline Phalacrocoracidae & 1 & 2 & 2.0 & \\
\hline INTRODUCED BIRDS & 2 & 9 & 4.5 & $2-7$ \\
\hline Columbiformes & 1 & 2 & 2.0 & \\
\hline Columbidae & 1 & 2 & 2.0 & \\
\hline Galliformes & 1 & 7 & 7.0 & \\
\hline Phasianidae & 1 & 7 & 7.0 & \\
\hline
\end{tabular}


TABLE 3. (Continued)

\begin{tabular}{lllll}
\hline & $\begin{array}{l}\text { Number of host } \\
\text { species }\end{array}$ & Host-louse associations & $\begin{array}{l}\text { Number of louse species per } \\
\text { host }\end{array}$ & Range \\
\hline CAPTIVE BIRDS & 5 & 11 & 2.2 & $1-5$ \\
Galliformes & $\mathbf{4}$ & $\mathbf{1 0}$ & $\mathbf{2 . 5}$ & $\mathbf{1 - 5}$ \\
$\quad 1$ & 1 & 1.0 & $1-\mathbf{5}$ \\
Numididae & 3 & 9 & 3.0 & $\mathbf{1}$ \\
Phasianidae & $\mathbf{1}$ & $\mathbf{1}$ & 1 \\
$\quad 1$ Strigiformes & 1 & 1 & \\
MAMMae & & $\mathbf{8}$ & $\mathbf{1 . 0}$ \\
Carnivora & $\mathbf{8}$ & $\mathbf{5}$ & $\mathbf{1 . 0}$ \\
Felidae & $\mathbf{5}$ & 1 & 1.0 \\
Mustelidae & 1 & 4 & 1.0 \\
Cetartiodactyla & 4 & $\mathbf{3}$ & 1.0 \\
Bovidae & $\mathbf{3}$ & 2 & 1.0 \\
Cervidae & 2 & 1 & 1.0 \\
\hline TOTAL & 1 & $\mathbf{3 6 6}$ & $\mathbf{2 . 0}$ \\
\hline
\end{tabular}

TABLE 4. List of louse genera with numbers of species and their host associations (including records at genus level) recorded from Slovakia.

\begin{tabular}{|c|c|c|c|c|c|}
\hline & $\begin{array}{l}\text { Number of } \\
\text { louse species }\end{array}$ & $\begin{array}{l}\text { Host-louse } \\
\text { associations }\end{array}$ & $\begin{array}{l}\text { Number of host } \\
\text { species per louse }\end{array}$ & Range & $\begin{array}{l}\text { New host-louse associations } \\
(* *=\text { worldwide })\end{array}$ \\
\hline Amblycera & 67 & 114 & 1.7 & $1-7$ & \\
\hline Laemobothriidae & 2 & 2 & 1.0 & & \\
\hline Laemobothrion & 2 & 2 & 1.0 & & \\
\hline Menoponidae & 60 & 100 & 1.6 & $1-7$ & \\
\hline Actornithophilus & 1 & 1 & 1.0 & & \\
\hline Amyrsidea & 2 & 2 & 1.0 & & \\
\hline Ardeiphilus & 1 & 1 & 1.0 & & \\
\hline Austromenopon & 4 & 4 & 1.0 & & \\
\hline Ciconiphilus & 2 & 2 & 1.0 & & \\
\hline Colpocephalum & 7 & 13 & 1.8 & $1-2$ & 2 \\
\hline Dennyus & 1 & 1 & 1.0 & & \\
\hline Eidmanniella & 1 & 1 & 1.0 & & \\
\hline Gruimenopon & 1 & 1 & 1.0 & & \\
\hline Heleonomus & 1 & 1 & 1.0 & & \\
\hline Holomenopon & 2 & $1+1$ & 1.0 & & $\begin{array}{l}* * \text { Holomenopon clypeilargum } \\
\text { ex Mergellus albellus }\end{array}$ \\
\hline Kurodaia & 4 & $4+1$ & 1.3 & $1-2$ & $\begin{array}{l}* * \text { Kurodaia }(\text { Conciella }) \\
\text { cryptostigmatia ex Strix } \\
\text { uralensis }\end{array}$ \\
\hline Machaerilaemus & 1 & 1 & 1.0 & & \\
\hline
\end{tabular}

......continued on the next page 
TABLE 4. (Continued)

\begin{tabular}{|c|c|c|c|c|c|}
\hline & $\begin{array}{l}\text { Number of } \\
\text { louse species }\end{array}$ & $\begin{array}{l}\text { Host-louse } \\
\text { associations }\end{array}$ & $\begin{array}{l}\text { Number of host } \\
\text { species per louse }\end{array}$ & Range & $\begin{array}{l}\text { New host-louse associations } \\
(* *=\text { worldwide) }\end{array}$ \\
\hline Menacanthus & 14 & $35+1$ & 2.6 & $1-8$ & $\begin{array}{l}8 \\
* * \text { Menacanthus eurysternus } \\
\text { ex Turdus iliacus }\end{array}$ \\
\hline Menopon & 2 & 2 & 1.0 & & \\
\hline Meromenopon & 1 & 1 & 1.0 & & \\
\hline Myrsidea & 10 & $10+1$ & 1.0 & & $\begin{array}{l}\text { ** Myrsidea thoracica ex } \\
\text { Turdus torquatus }\end{array}$ \\
\hline Nosopon & 1 & 1 & 1.0 & & \\
\hline Pseudomenopon & 2 & 3 & 1.5 & $1-2$ & 1 \\
\hline Trinoton & 2 & 10 & 5.0 & $3-7$ & 2 \\
\hline Ricinidae & 5 & 13 & 2.6 & $1-5$ & \\
\hline Ricinus & 5 & 13 & 2.6 & $1-5$ & 1 \\
\hline Ischnocera & 196 & 252 & 1.3 & 1-9 & \\
\hline Philopteridae & 188 & 244 & 1.3 & 1-9 & \\
\hline Acronirmus & 1 & 2 & 2.0 & & 1 \\
\hline Alcedoffula & 1 & 1 & 1.0 & & \\
\hline Anaticola & 4 & 9 & 2.3 & $1-3$ & \\
\hline Anatoecus & 1 & 9 & 9.0 & & 1 \\
\hline Aquanirmus & 3 & 3 & 1.0 & & \\
\hline Ardeicola & 5 & 5 & 1.0 & & \\
\hline Brueelia & 21 & 27 & 1.3 & $1-2$ & \\
\hline Campanulotes & 2 & 2 & 1.0 & & \\
\hline Capraiella & 1 & 1 & 1.0 & & \\
\hline Carduiceps & 1 & 1 & 1.0 & & \\
\hline Cincloecus & 1 & 1 & 1.0 & & \\
\hline Coloceras & 2 & 2 & 1.0 & & \\
\hline Columbicola & 3 & 4 & 1.3 & $1-2$ & \\
\hline Corvonirmus & 2 & 2 & 1.0 & & \\
\hline Craspedonirmus & 1 & 1 & 1.0 & & \\
\hline Craspedorrhynchus & 7 & 9 & 1.3 & $1-2$ & \\
\hline Cuclotogaster & 2 & 3 & 1.0 & & \\
\hline Cuculicola & 1 & 1 & 1.0 & & \\
\hline Cuculoecus & 1 & 1 & 1.0 & & \\
\hline Cummingsiella & 2 & 2 & 1.0 & & \\
\hline Degeeriella & 9 & 16 & 1.8 & $1-4$ & \\
\hline Esthiopterum & 1 & 1 & 1.0 & & \\
\hline Falcolipeurus & 2 & 2 & 1.0 & & \\
\hline Fulicoffula & 3 & 3 & 1.0 & & 1 \\
\hline Goniocotes & 3 & 3 & 1.0 & & \\
\hline Goniodes & 6 & 6 & 1.0 & & \\
\hline
\end{tabular}

......continued on the next page 
TABLE 4. (Continued)

\begin{tabular}{|c|c|c|c|c|c|}
\hline & $\begin{array}{l}\text { Number of } \\
\text { louse species }\end{array}$ & $\begin{array}{l}\text { Host-louse } \\
\text { associations }\end{array}$ & $\begin{array}{l}\text { Number of host } \\
\text { species per louse }\end{array}$ & Range & $\begin{array}{l}\text { New host-louse associations } \\
(* *=\text { worldwide) }\end{array}$ \\
\hline Guimaraesiella & 2 & 2 & 1.0 & & \\
\hline Hecatrishula & 1 & 2 & 2.0 & & \\
\hline Ibidoecus & 2 & 2 & 1.0 & & \\
\hline Lagopoecus & 4 & 4 & 1.0 & & \\
\hline Lipeurus & 3 & 3 & 1.0 & & \\
\hline Lunaceps & 1 & 1 & 1.0 & & \\
\hline Maculinirmus & 1 & 1 & 1.0 & & \\
\hline Meropoecus & 1 & 1 & 1.0 & & \\
\hline Meropsiella & 1 & 1 & 1.0 & & \\
\hline Mulcticola & 1 & 1 & 1.0 & & \\
\hline Neophilopterus & 2 & 2 & 1.0 & & \\
\hline Olivinirmus & 1 & 1 & 1.0 & & \\
\hline Ornithobius & 1 & 1 & 1.0 & & \\
\hline Otidoecus & 1 & 1 & 1.0 & & \\
\hline Oxylipeurus & 3 & 3 & 1.0 & & \\
\hline Pectinopygus & 1 & 1 & 1.0 & & \\
\hline Penenirmus & 8 & 12 & 1.5 & $1-4$ & \\
\hline Philopterus & 33 & 50 & 1.5 & $1-5$ & \\
\hline Picicola & 2 & 3 & 1.5 & $1-2$ & \\
\hline Quadraceps & 10 & 10 & 1.0 & & \\
\hline Rallicola & 3 & 3 & 1.0 & & \\
\hline Rhynonirmus & 1 & 1 & 1.0 & & \\
\hline Rostrinirmus & 3 & 3 & 1.0 & & \\
\hline Saemundssonia & 5 & 6 & 1.2 & $1-2$ & \\
\hline Strigiphilus & 8 & 8 & 1.0 & & \\
\hline Sturnidoecus & 2 & 2 & 1.0 & & \\
\hline Turdinirmus & 1 & 1 & 1.0 & & \\
\hline Upupicola & 1 & 1 & 1.0 & & \\
\hline Trichodectidae & 8 & 8 & 1.0 & & \\
\hline Bovicola & 2 & 2 & 1.0 & & \\
\hline Damalinia & 1 & 1 & 1.0 & & \\
\hline Felicola & 1 & 1 & 1.0 & & \\
\hline Stachiella & 3 & 3 & 1.0 & & \\
\hline Trichodectes & 1 & 1 & 1.0 & & \\
\hline TOTAL & 263 & 366 & 1.4 & 1-9 & 20 \\
\hline
\end{tabular}


TABLE 5. List of host-louse associations of chewing lice from Slovakia.

$\#=$ first record of louse species from Slovakia; $\S=$ new host-louse association for Slovakia; $* *=$ new host-louse association worldwide. Host taxa listed in alphabetical sequence by Order.

\begin{tabular}{l}
\hline HOSTS \\
\hline AVES \\
Accipitriformes \\
Accipitridae \\
$\quad$ Accipiter gentilis (Linnaeus, 1758) \\
Accipiter nisus (Linnaeus, 1758) \\
Aquila chrysaetos (Linnaeus, 1758)
\end{tabular}

Aquila heliaca Savigny, 1809

Buteo buteo (Linnaeus, 1758)

Buteo lagopus (Pontoppidan, 1763)

Buteo rufinus (Cretzschmar, 1829)

Circaetus gallicus (Gmelin, 1788)

Circus aeruginosus (Linnaeus, 1758)

Circus cyaneus (Linnaeus, 1766)

Circus macrourus (Gmelin, 1770)

Clanga pomarina (Brehm, 1831)

Haliaeetus albicilla (Linnaeus, 1758)

Milvus migrans (Boddaert, 1783)

Pernis apivorus (Linnaeus, 1758)

\section{Pandionidae}

Pandion haliaetus (Linnaeus, 1758)
LICE

Craspedorrhynchus haematopus (Scopoli, 1763)

\#

Degeeriella vagans (Giebel, 1874)

Degeeriella nisus (Giebel, 1866)

Colpocephalum impressum Rudow, 1866

Craspedorrhynchus aquilinus (Denny, 1842)

Degeeriella fulva (Giebel, 1874)

Falcolipeurus suturalis (Rudow, 1869)

Colpocephalum sp.

Craspedorrhynchus sp.

Colpocephalum nanum Piaget, 1890

Craspedorrhynchus platystomus (Burmeister, 1838)

Degeeriella fulva (Giebel, 1874)

Kurodaia (Kurodaia) fulvofasciata (Piaget, 1880)

Colpocephalum nanum Piaget, 1890

Craspedorrhynchus dilatatus (Rudow, 1869)

Degeeriella fulva (Giebel, 1874)

Craspedorrhynchus platystomus (Burmeister, 1838)

Degeeriella fulva (Giebel, 1874)

Degeeriella leucopleura (Nitzsch, 1874)

Colpocephalum turbinatum Denny, 1842

Degeeriella fusca (Denny, 1842)

Degeeriella fusca (Denny, 1842)

Degeeriella fusca (Denny, 1842)

Colpocephalum impressum Rudow, 1866

Craspedorrhynchus naevius (Giebel, 1861)

Degeeriella aquilarum Eichler, 1943

Colpocephalum flavescens (Haan, 1829)

Craspedorrhynchus macrocephalus (Nitzsch, 1874)

Degeeriella discocephalus (Burmeister, 1838)

Falcolipeurus sulcifrons (Denny, 1842)

Degeeriella regalis (Giebel, 1866)

Craspedorrhynchus melittoscopus (Nitzsch, 1874)

Kurodaia (Kurodaia) haliaeeti (Denny, 1842)

\section{Anseriformes \\ Anatidae}

Anas acuta Linnaeus, 1758

Trinoton querquedulae (Linnaeus, 1758)

Anas crecca Linnaeus, 1758

Trinoton querquedulae (Linnaeus, 1758)

......continued on the next page 


\begin{tabular}{|c|c|c|}
\hline HOSTS & LICE & \\
\hline \multirow[t]{3}{*}{ Anas platyrhynchos Linnaeus, 1758} & Anaticola crassicornis (Scopoli, 1763) & \\
\hline & Anatoecus dentatus (Scopoli, 1763) & \\
\hline & Trinoton querquedulae (Linnaeus, 1758) & \\
\hline Anser albifrons (Scopoli, 1769) & Anaticola anseris (Linnaeus, 1758) & \\
\hline \multirow[t]{4}{*}{ Anser brachyrhynchus Baillon, 1834} & Anaticola anseris (Linnaeus, 1758) & \\
\hline & Anatoecus sp. & \\
\hline & Ciconiphilus pectiniventris (Harrison, 1916) & $\#$ \\
\hline & Trinoton anserinum (J.C. Fabricius, 1805) & \\
\hline \multirow[t]{3}{*}{ Anser fabalis (Latham, 1787) } & Anaticola anseris (Linnaeus, 1758) & \\
\hline & Anatoecus dentatus (Scopoli, 1763) & \\
\hline & Trinoton anserinum (J.C. Fabricius, 1805) & \\
\hline \multirow[t]{2}{*}{ Aythya ferina (Linnaeus, 1758) } & Anatoecus dentatus (Scopoli, 1763) & $\S$ \\
\hline & Trinoton querquedulae (Linnaeus, 1758) & $\S$ \\
\hline \multirow[t]{2}{*}{ Aythya fuligula (Linnaeus, 1758) } & Anaticola mergiserrati (De Geer, 1778) & $\#$ \\
\hline & Trinoton querquedulae (Linnaeus, 1758) & $\S$ \\
\hline \multirow[t]{2}{*}{ Branta ruficollis (Pallas, 1769) } & Anaticola beieri Eichler, 1954a & \\
\hline & Anatoecus dentatus (Scopoli, 1763) & \\
\hline \multirow[t]{3}{*}{ Cygnus olor (Gmelin, 1789) } & Anatoecus dentatus (Scopoli, 1763) & \\
\hline & Ornithobius bucephalus (Giebel, 1874) & \# \\
\hline & Trinoton anserinum (J.C. Fabricius, 1805) & \\
\hline \multirow[t]{4}{*}{ Mergellus albellus (Linnaeus, 1758) } & Anaticola mergiserrati (De Geer, 1778) & $\#$ \\
\hline & Anatoecus dentatus (Scopoli, 1763) & \\
\hline & Holomenopon clypeilargum Eichler, 1943 & $\S * *$ \\
\hline & Trinoton querquedulae (Linnaeus, 1758) & \\
\hline Mergus merganser Linnaeus, 1758 & Anatoecus dentatus (Scopoli, 1763) & \\
\hline Spatula clypeata (Linnaeus, 1758) & Anaticola crassicornis (Scopoli, 1763) & \\
\hline \multirow[t]{3}{*}{ Spatula querquedula (Linnaeus, 1758) } & Anaticola crassicornis (Scopoli, 1763) & \\
\hline & Anatoecus dentatus (Scopoli, 1763) & \\
\hline & Trinoton querquedulae (Linnaeus, 1758) & \\
\hline Tadorna tadorna (Linnaeus, 1758) & Holomenopon tadornae (Gervais, 1844) & \\
\hline
\end{tabular}

\section{Apodiformes \\ Apodidae}

Apus apus (Linnaeus, 1758)

Dennyus hirundinis (Linnaeus, 1761)

\section{Bucerotiformes}

Upupidae

Upupa epops Linnaeus, 1758

Menacanthus fertilis (Nitzsch, 1866)

Upupicola upupae (Schrank, 1803)

\section{Caprimulgiformes}

\section{Caprimulgidae}

Caprimulgus europaeus Linnaeus, 1758

Mulcticola hypoleucus (Denny, 1842)

......continued on the next page 


HOSTS LICE

\section{Charadriiformes}

\section{Charadriidae}

Charadrius dubius Scopoli, 1786

Charadrius hiaticula Linnaeus, 1758

Vanellus vanellus (Linnaeus, 1758)

\section{Laridae}

Chlidonias leucopterus (Temminck, 1815)

Chlidonias niger (Linnaeus, 1758)

Chroicocephalus ridibundus (Linnaeus, 1766)

Larus argentatus Pontoppidan, 1763

Sterna hirundo Linnaeus, 1758

\section{Scolopacidae}

Actitis hypoleucos (Linnaeus, 1758)

Calidris pugnax (Linnaeus, 1758)

Numenius arquata (Linnaeus, 1758)

Scolopax rusticola Linnaeus, 1758

Tringa erythropus (Pallas, 1764)

Tringa ochropus Linnaeus, 1758

\section{Ciconiiformes}

\section{Ciconiidae}

Ciconia ciconia (Linnaeus, 1758)

Ciconia nigra (Linnaeus, 1758)
Austromenopon aegialitidis (Durrant, 1906)

Quadraceps bicuspis (Nitzsch, 1874)

Quadraceps fissus (Burmeister, 1838)

Actornithophilus gracilis (Piaget, 1880)

Quadraceps junceus (Scopoli, 1763)

Quadraceps anagrapsus (Nitzsch [in Giebel], 1866) Saemundssonia (Saemundssonia) lobaticeps

(Giebel, 1874)

Quadraceps phaeonotus (Nitzsch [in Giebel], 1866)

Austromenopon transversum (Denny, 1842)

Quadraceps punctatus punctatus (Burmeister, 1838)

Saemundssonia (Saemundssonia) lari

(O. Fabricius, 1780)

Saemundssonia (Saemundssonia) sp.

Quadraceps sellatus (Burmeister, 1838)

Saemundssonia (Saemundssonia) sternae (Linnaeus, 1758)

Quadraceps ravus (Kellogg, 1899)

Carduiceps scalaris (Piaget, 1880)

Saemundssonia (Saemundssonia) tringae

(O. Fabricius, 1780)

Austromenopon crocatum (Nitzsch [in Giebel], 1866)

Cummingsiella ovalis (Scopoli, 1763)

Lunaceps numenii (Denny, 1842)

Cummingsiella aurea Hopkins, 1949

Rhynonirmus helvolus (Burmeister, 1838)

Austromenopon decorosum Złotorzycka, 1968

Quadraceps furvus (Burmeister, 1838)

Quadraceps ochropi (Denny, 1842)

Ardeicola ciconiae (Linnaeus, 1758)

Colpocephalum zebra Burmeister, 1838

Neophilopterus incompletus (Denny, 1842)

Neophilopterus tricolor (Burmeister, 1838)

\section{Columbiformes}

......continued on the next page 


\begin{tabular}{|c|c|c|}
\hline HOSTS & LICE & \\
\hline \multicolumn{3}{|l|}{ Columbidae } \\
\hline \multirow[t]{2}{*}{ Columba livia Gmelin, 1789_introduced species } & Campanulotes compar (Burmeister, 1838) & \\
\hline & Columbicola columbae (Linnaeus, 1758) & \\
\hline \multirow[t]{3}{*}{ Columba palumbus Linnaeus, 1758} & Campanulotes bidentatus (Scopoli, 1763) & $\#$ \\
\hline & Coloceras damicorne (Nitzsch, 1866) & \\
\hline & Columbicola claviformis (Denny, 1842) & $\#$ \\
\hline \multirow[t]{2}{*}{ Streptopelia decaocto (Frivaldszky, 1838) } & Coloceras piageti (Johnston \& Harrison, 1912) & \\
\hline & Columbicola bacillus (Giebel, 1866) & \\
\hline Streptopelia turtur (Linnaeus, 1758) & Columbicola bacillus (Giebel, 1866) & \\
\hline \multicolumn{3}{|l|}{ Coraciiformes } \\
\hline \multicolumn{3}{|l|}{ Alcedinidae } \\
\hline Alcedo atthis (Linnaeus, 1758) & Alcedoffula alcedinis (Denny, 1842) & \\
\hline \multicolumn{3}{|l|}{ Coraciidae } \\
\hline \multirow[t]{2}{*}{ Coracias garrulus Linnaeus, 1758} & Capraiella subcuspidata (Burmeister, 1838) & \\
\hline & Laemobothrion sp. (accidental straggler) & \\
\hline \multicolumn{3}{|l|}{ Meropidae } \\
\hline \multirow[t]{3}{*}{ Merops apiaster Linnaeus, 1758} & Meromenopon meropis Clay \& Meinertzhagen, 1941 & \\
\hline & Meropoecus meropis (Denny, 1842) & \\
\hline & Meropsiella apiastri (Denny, 1842) & \\
\hline \multicolumn{3}{|l|}{ Cuculiformes } \\
\hline \multicolumn{3}{|l|}{ Cuculidae } \\
\hline \multirow[t]{2}{*}{ Cuculus canorus Linnaeus, 1758} & Cuculicola latirostris (Burmeister, 1838) & \\
\hline & Cuculoecus latifrons (Denny, 1842) & \\
\hline \multicolumn{3}{|l|}{ Falconiformes } \\
\hline \multicolumn{3}{|l|}{ Falconidae } \\
\hline \multirow[t]{2}{*}{ Falco cherrug Gray, 1834} & Colpocephalum sp. & \\
\hline & Degeeriella rufa (Burmeister, 1838) & \\
\hline \multirow[t]{4}{*}{ Falco tinnunculus Linnaeus, 1758} & Colpocephalum sp. & \\
\hline & Degeeriella rufa (Burmeister, 1838) & \\
\hline & $\begin{array}{l}\text { Laemobothrion (Laemobothrion) tinnunculi (Linnaeus, } \\
1758 \text { ) }\end{array}$ & \\
\hline & Nosopon lucidum (Rudow, 1869) & $\#$ \\
\hline Falco vespertinus Linnaeus, 1766 & Degeeriella rufa (Burmeister, 1838) & \\
\hline \multicolumn{3}{|l|}{ Galliformes } \\
\hline \multicolumn{3}{|l|}{ Numididae } \\
\hline Numida meleagris (Linnaeus, 1758)_ captive bird & Lipeurus numidae (Denny, 1842) & \\
\hline \multicolumn{3}{|l|}{ Phasianidae } \\
\hline Chrysolophus pictus (Linnaeus, 1758)_captive bird & Oxylipeurus mesopelios (Nitzsch [in Giebel], 1866) & \\
\hline Gallus gallus (Linnaeus, 1758) — captive bird & $\begin{array}{l}\text { Cuclotogaster heterographus (Nitzsch [in Giebel], } \\
1866)\end{array}$ & $\#$ \\
\hline
\end{tabular}




\begin{tabular}{|c|c|c|}
\hline HOSTS & LICE & \\
\hline & Goniocotes gallinae (De Geer, 1778) & \# \\
\hline & Goniodes dissimilis Denny, 1842 & \\
\hline & Menacanthus stramineus (Nitzsch, 1818) & \# \\
\hline & Menopon gallinae (Linnaeus, 1758) & \# \\
\hline \multirow[t]{2}{*}{ Lyrurus tetrix (Linnaeus, 1758) } & Goniodes tetraonis (Linnaeus, 1761) & \\
\hline & Lagopoecus lyrurus Clay, 1938 & \\
\hline \multirow[t]{3}{*}{ Pavo cristatus Linnaeus, 1758 - captive bird } & Amyrsidea phaeostoma (Nitzsch [in Giebel], 1866) & \\
\hline & Goniodes pavonis (Linnaeus, 1758) & \\
\hline & Lipeurus pavo Clay, 1938 & \\
\hline \multirow[t]{3}{*}{ Perdix perdix (Linnaeus, 1758) } & $\begin{array}{l}\text { Cuclotogaster heterogrammicus (Nitzsch [in Giebel], } \\
\text { 1866) }\end{array}$ & \\
\hline & Goniodes dispar Burmeister, 1838 & \# \\
\hline & Menopon pallens Clay, 1949 & \\
\hline \multirow{7}{*}{$\begin{array}{l}\text { Phasianus colchicus Linnaeus, 1758-introduced } \\
\text { species }\end{array}$} & Amyrsidea perdicis (Denny, 1842) & \\
\hline & $\begin{array}{l}\text { Cuclotogaster heterographus (Nitzsch [in Giebel], } \\
\text { 1866) }\end{array}$ & \# \\
\hline & Goniocotes chrysocephalus Giebel, 1874 & \\
\hline & Goniodes colchici Denny, 1842 & \\
\hline & Lagopoecus colchicus Emerson, 1949 & \\
\hline & Lipeurus maculosus Clay, 1938 & \\
\hline & Oxylipeurus colchicus Clay, 1938 & \\
\hline \multirow[t]{3}{*}{ Tetrao urogallus Linnaeus, 1758} & Goniodes bituberculatus Rudow, 1869 & \\
\hline & Lagopoecus pallidovittatus (Grube, 1851) & \\
\hline & Oxylipeurus tetraonis (Grube, 1851) & \\
\hline \multirow[t]{2}{*}{ Tetrastes bonasia (Linnaeus, 1758) } & Goniocotes megalocephalus Uchida, 1916 & \# \\
\hline & Lagopoecus tetrastei Bechet, 1963 & \\
\hline \multicolumn{3}{|l|}{ Gaviiformes } \\
\hline \multicolumn{3}{|l|}{ Gaviidae } \\
\hline Gavia arctica (Linnaeus, 1758) & Craspedonirmus colymbinus (Denny, 1842) & \\
\hline \multicolumn{3}{|l|}{ Gruiformes } \\
\hline \multicolumn{3}{|l|}{ Gruidae } \\
\hline \multirow[t]{5}{*}{ Grus grus (Linnaeus, 1758) } & Esthiopterum gruis (Linnaeus, 1758) & \\
\hline & Gruimenopon longum (Giebel, 1874) & \\
\hline & Heleonomus macilentus (Nitzsch [in Giebel], 1866) & \\
\hline & Saemundssonia (Saemundssonia) integer & \\
\hline & (Nitzsch [in Giebel], 1866) & \\
\hline \multicolumn{3}{|l|}{ Rallidae } \\
\hline \multirow[t]{3}{*}{ Fulica atra Linnaeus, 1758} & Fulicoffula lurida (Nitzsch, 1818) & \\
\hline & $\begin{array}{l}\text { Laemobothrion (Eulaemobothrion) atrum } \\
\text { (Nitzsch, 1818) }\end{array}$ & \\
\hline & Pseudomenopon pilosum (Scopoli, 1763) & $\S$ \\
\hline
\end{tabular}

......continued on the next page 


\begin{tabular}{ll}
\hline HOSTS & LICE \\
\hline \multirow{2}{*}{ Gallinula chloropus (Linnaeus, 1758) } & Rallicola (Rallicola) fulicae (Denny, 1842) \\
& Fulicoffula gallinula Carriker, 1953 \\
& Pseudomenopon pilosum (Scopoli, 1763) \\
Rallus aquaticus Linnaeus, 1758 & Rallicola (Rallicola) minutus (Nitzsch [in Giebel], \\
& 1866) \\
& Fulicoffula sp. \\
& Rallicola (Rallicola) cuspidatus (Scopoli, 1763)
\end{tabular}

\section{Otidiformes}

\section{Otididae}

Otis tarda Linnaeus, 1758

Otidoecus turmalis (Denny, 1842)

\section{Passeriformes}

\section{Acrocephalidae}

Acrocephalus arundinaceus (Linnaeus, 1758)

Acrocephalus melanopogon (Temminck, 1823)

Acrocephalus schoenobaenus (Linnaeus, 1758)

Acrocephalus scirpaceus (Hermann, 1804)

\section{Aegithalidae}

Aegithalos caudatus (Linnaeus, 1758)

\section{Alaudidae}

Alauda arvensis Linnaeus, 1758

Galerida cristata (Linnaeus, 1758)

\section{Certhiidae}

Certhia familiaris Linnaeus, 1758

\section{Cinclidae}

Cinclus cinclus (Linnaeus, 1758)

\section{Corvidae}

Coloeus monedula (Linnaeus, 1758)

Corvus corax Linnaeus, 1758

Philopterus fedorenkoae (Mey, 1983)

Philopterus acrocephalus Carriker, 1949

Brueelia vaneki Balát, 1981

Menacanthus curuccae (Schrank, 1776)

Philopterus sp.

Brueelia sp.

Menacanthus curuccae (Schrank, 1776)

Philopterus sp.

Penenirmus pari (Denny, 1842)

Brueelia parviguttata (Blagoveshtchensky, 1940)

Menacanthus alaudae (Schrank, 1776)

Philopterus stadleri (Eichler, 1959)

Philopterus alexanderkoenigi (Eichler, 1953b) \#

Ricinus serratus (Durrant, 1906)

Penenirmus gulosus (Nitzsch, 1866)

Cincloecus cincli (Denny, 1842)

Myrsidea franciscoloi Conci, 1942

Hecatrishula varia (Burmeister, 1838)

Menacanthus eurysternus (Burmeister, 1838)

Menacanthus gonophaeus (Burmeister, 1838)

Myrsidea anathorax (Nitzsch, 1866)

Philopterus guttatus (Denny, 1842)

Colpocephalum fregili Denny, 1842

Menacanthus gonophaeus (Burmeister, 1838) 


\begin{tabular}{|c|c|c|}
\hline HOSTS & LICE & \\
\hline & Philopterus corvi (Linnaeus, 1758) & \\
\hline \multirow[t]{4}{*}{ Corvus cornix Linnaeus, 1758} & Corvonirmus uncinosus (Burmeister, 1838) & \\
\hline & Menacanthus eurysternus (Burmeister, 1838) & \\
\hline & Myrsidea cornicis (De Geer, 1778) & \\
\hline & Philopterus ocellatus (Scopoli, 1763) & \\
\hline \multirow[t]{6}{*}{ Corvus frugilegus Linnaeus, 1758} & Colpocephalum fregili Denny, 1842 & $\S$ \\
\hline & Corvonirmus tasniemae (Ansari, 1957) & \\
\hline & Hecatrishula varia (Burmeister, 1838) & \# \\
\hline & Menacanthus gonophaeus (Burmeister, 1838) & \\
\hline & Myrsidea isostoma (Nitzsch, 1866) & \# \\
\hline & Philopterus atratus (Nitzsch, 1818) & \\
\hline \multirow[t]{2}{*}{ Garrulus glandarius (Linnaeus, 1758) } & Olivinirmus glandarii (Denny, 1842) & \\
\hline & Philopterus garruli Boisduval \& Lacordaire, 1935 & \\
\hline \multirow[t]{2}{*}{ Nucifraga caryocatactes (Linnaeus, 1758) } & Menacanthus merisuoi Eichler, 1953c & \# \\
\hline & Philopterus crassipes (Burmeister, 1838) & \\
\hline \multirow[t]{2}{*}{ Pica pica (Linnaeus, 1758) } & Myrsidea picae (Linnaeus, 1758) & \\
\hline & Philopterus picae (Denny, 1842) & \\
\hline \multicolumn{3}{|l|}{ Emberizidae } \\
\hline \multirow[t]{3}{*}{ Emberiza citrinella Linnaeus, 1758} & Brueelia delicata (Nitzsch [in Giebel], 1866) & \\
\hline & Philopterus citrinellae (Schrank, 1776) & \\
\hline & Ricinus fringillae De Geer, 1778 & \\
\hline \multirow[t]{4}{*}{ Emberiza schoeniclus (Linnaeus, 1758) } & Brueelia blagovescenskyi Balát, 1955b & \# \\
\hline & Menacanthus chrysophaeus (Kellogg, 1896) & \\
\hline & Philopterus citrinellae (Schrank, 1776) & \\
\hline & Ricinus fringillae De Geer, 1778 & \\
\hline \multicolumn{3}{|l|}{ Fringillidae } \\
\hline Carduelis carduelis (Linnaeus, 1758) & Menacanthus alaudae (Schrank, 1776) & \\
\hline Carpodacus erythrinus (Pallas, 1770) & Rostrinirmus carpodaci Balát, 1981 & \\
\hline \multirow[t]{2}{*}{ Chloris chloris (Linnaeus, 1758) } & Brueelia breueri Balát, 1955b & \\
\hline & Philopterus citrinellae (Schrank, 1776) & \\
\hline Coccothraustes coccothraustes (Linnaeus, 1758) & Brueelia juno (Giebel, 1874) & \\
\hline \multirow[t]{3}{*}{ Fringilla coelebs Linnaeus, 1758} & Brueelia kluzi Balát, 1955 & \\
\hline & Philopterus fortunatus (Złotorzycka, 1964) & \\
\hline & Ricinus fringillae De Geer, 1778 & \\
\hline Fringilla montifringilla Linnaeus, 1758 & Philopterus rapax (Złotorzycka, 1964) & \\
\hline Linaria cannabina (Linnaeus, 1758) & Brueelia stadleri Eichler, 1954b & \\
\hline Loxia curvirostra Linnaeus, 1758 & Philopterus curvirostrae (Schrank, 1776) & \\
\hline \multirow[t]{3}{*}{ Pyrrhula pyrrhula (Linnaeus, 1758) } & Brueelia pyrrhularum Eichler, 1954b & \\
\hline & Philopterus citrinellae (Schrank, 1776) & \\
\hline & Philopterus fringillae (Scopoli, 1772) & \\
\hline \multirow[t]{2}{*}{ Spinus spinus (Linnaeus, 1758) } & Brueelia chrysomytris (Blagoveshtchenky, 1940) & \\
\hline & Philopterus citrinellae (Schrank, 1776) & \\
\hline
\end{tabular}

......continued on the next page 


\begin{tabular}{|c|c|c|}
\hline HOSTS & LICE & \\
\hline \multirow[t]{2}{*}{ Delichon urbicum (Linnaeus, 1758) } & Acronirmus gracilis (Burmeister, 1838) & \\
\hline & Philopterus excisus Nitzsch, 1818 & \\
\hline \multirow[t]{3}{*}{ Hirundo rustica Linnaeus, 1758} & Acronirmus gracilis (Burmeister, 1838) & $\S$ \\
\hline & Myrsidea rustica (Giebel, 1874) & $\#$ \\
\hline & Philopterus microsomaticus Tandan, 1955 & \\
\hline \multirow[t]{3}{*}{ Riparia riparia (Linnaeus, 1758) } & Machaerilaemus clayae (Balát, 1966) & $\#$ \\
\hline & $\begin{array}{l}\text { Myrsidea latifrons (Carriker [in Carriker \& Shull], } \\
\text { 1910) }\end{array}$ & $\#$ \\
\hline & Philopterus microsomaticus Tandan, 1955 & \\
\hline \multicolumn{3}{|l|}{ Laniidae } \\
\hline \multirow[t]{2}{*}{ Lanius collurio Linnaeus, 1758} & Menacanthus camelinus (Nitzsch, 1874) & $\S$ \\
\hline & Philopterus coarctatus (Scopoli, 1763) & \\
\hline \multirow[t]{3}{*}{ Lanius excubitor Linnaeus, 1758} & Brueelia imponderabilica Eichler, 1954b & \\
\hline & Menacanthus camelinus (Nitzsch, 1874) & \\
\hline & Philopterus coarctatus (Scopoli, 1763) & \\
\hline \multirow[t]{3}{*}{ Lanius minor Gmelin, 1788} & Brueelia sp. & \\
\hline & Menacanthus camelinus (Nitzsch, 1874) & \\
\hline & Philopterus coarctatus (Scopoli, 1763) & \\
\hline \multicolumn{3}{|l|}{ Locustellidae } \\
\hline Locustella fluviatilis (Wolf, 1810) & Sturnidoecus tulackovae (Balát, 1981) & \\
\hline \multirow[t]{2}{*}{ Locustella luscinioides (Savi, 1824) } & Brueelia locustellae Fedorenko, 1975 & $\#$ \\
\hline & Menacanthus obrteli Balát, 1981 & \\
\hline \multicolumn{3}{|l|}{ Motacillidae } \\
\hline \multirow[t]{3}{*}{ Anthus spinoletta (Linnaeus, 1758) } & Menacanthus pusillus (Nitzsch, 1866) & \\
\hline & Ricinus fringillae De Geer, 1778 & \\
\hline & Philopterus hanzaki Balát, 1955a & \\
\hline Anthus trivialis (Linnaeus, 1758) & Philopterus sp. & \\
\hline \multirow[t]{2}{*}{ Motacilla alba Linnaeus, 1758} & Brueelia kratochvili Balát, 1958 & \\
\hline & Philopterus passerinus (Denny, 1842) & \\
\hline Motacilla cinerea Tunstall, 1771 & Menacanthus pusillus (Nitzsch, 1866) & \\
\hline \multirow[t]{2}{*}{ Motacilla flava Linnaeus, 1758} & Brueelia kratochvili Balát, 1958 & \\
\hline & Menacanthus pusillus (Nitzsch, 1866) & $\S$ \\
\hline \multicolumn{3}{|l|}{ Muscicapidae } \\
\hline Erithacus rubecula (Linnaeus, 1758) & Ricinus rubeculae (Schrank, 1776) & \\
\hline Ficedula parva (Bechstein, 1792) & Philopterus markevichi Fedorenko \& Volkov, 1977 & \\
\hline Oenanthe oenanthe (Linnaeus, 1758) & Ricinus sp. & \\
\hline \multirow[t]{3}{*}{ Saxicola rubetra (Linnaeus, 1758) } & Brueelia sp. & \\
\hline & Penenirmus nirmoideus (Nitzsch, 1874) & \\
\hline & Philopterus sp. & \\
\hline \multicolumn{3}{|l|}{ Oriolidae } \\
\hline \multirow[t]{3}{*}{ Oriolus oriolus (Linnaeus, 1758) } & Maculinirmus mundus (Nitzsch [in Giebel], 1866) & \\
\hline & Philopterus ornatus (Nitzsch, 1866) & \\
\hline & Ricinus dolichocephalus (Scopoli, 1763) & \\
\hline
\end{tabular}




\begin{tabular}{|c|c|c|}
\hline HOSTS & LICE & \\
\hline \multicolumn{3}{|l|}{ Panuridae } \\
\hline \multirow[t]{2}{*}{ Panurus biarmicus (Linnaeus, 1758) } & Menacanthus sp. & $\#$ \\
\hline & Penenirmus visendus (Złotorzycka, 1964) & $\#$ \\
\hline \multicolumn{3}{|l|}{ Paridae } \\
\hline Cyanistes caeruleus (Linnaeus, 1758) & Menacanthus sinuatus (Burmeister, 1838) & \\
\hline \multirow[t]{3}{*}{ Parus major Linnaeus, 1758} & Menacanthus sinuatus (Burmeister, 1838) & \\
\hline & Philopterus thuringiacus (Mey, 1988) & \\
\hline & Rostrinirmus hudeci Balát, 1981 & \\
\hline \multirow[t]{2}{*}{ Periparus ater (Linnaeus, 1758) } & Menacanthus sinuatus (Burmeister, 1838) & \\
\hline & Philopterus peripariphilus (Mey, 1988) & \\
\hline \multirow[t]{2}{*}{ Poecile palustris (Linnaeus, 1758) } & Menacanthus sinuatus (Burmeister, 1838) & \\
\hline & Philopterus pallescens (Denny, 1842) & \\
\hline \multicolumn{3}{|l|}{ Passeridae } \\
\hline \multirow[t]{2}{*}{ Passer domesticus (Linnaeus, 1758) } & Brueelia cyclothorax (Burmeister, 1838) & \\
\hline & Philopterus fringillae (Scopoli, 1772) & \\
\hline \multirow[t]{5}{*}{ Passer montanus (Linnaeus, 1758) } & Brueelia cyclothorax (Burmeister, 1838) & \\
\hline & Menacanthus eurysternus (Burmeister, 1838) & \\
\hline & Myrsidea quadrifasciata quadrifasciata (Piaget, 1880) & \\
\hline & Philopterus fringillae (Scopoli, 1772) & \\
\hline & Rostrinirmus ruficeps (Nitzsch [in Giebel], 1866) & \\
\hline \multicolumn{3}{|l|}{ Phylloscopidae } \\
\hline Phylloscopus collybita (Vieillot, 1817) & Menacanthus agilis (Nitzsch, 1866) & \# \\
\hline Phylloscopus trochilus (Linnaeus, 1758) & Menacanthus agilis (Nitzsch, 1866) & \# \\
\hline \multicolumn{3}{|l|}{ Prunellidae } \\
\hline \multirow[t]{2}{*}{ Prunella collaris (Scopoli, 1769) } & Philopterus emiliae Balát, 1955a & \\
\hline & Ricinus fringillae De Geer, 1778 & \\
\hline Prunella modularis (Linnaeus, 1758) & Philopterus modularis (Denny, 1842) & \\
\hline \multicolumn{3}{|l|}{ Regulidae } \\
\hline Regulus regulus (Linnaeus, 1758) & Philopterus gustafssoni Najer et al., 2020 & \# \\
\hline \multicolumn{3}{|l|}{ Remizidae } \\
\hline \multirow[t]{2}{*}{ Remiz pendulinus (Linnaeus, 1758) } & Brueelia balati (Krištofik, 1999) & \\
\hline & Menacanthus eurysternus (Burmeister, 1838) & \\
\hline \multicolumn{3}{|l|}{ Sittidae } \\
\hline \multirow[t]{2}{*}{ Sitta europaea Linnaeus, 1758} & Brueelia conocephalus (Blagoveshtchensky, 1940) & \\
\hline & Philopterus sittae Fedorenko, 1978 & \\
\hline \multicolumn{3}{|l|}{ Sturnidae } \\
\hline \multirow[t]{4}{*}{ Sturnus vulgaris Linnaeus, 1758} & Brueelia nebulosa (Burmeister, 1838) & \\
\hline & Menacanthus eurysternus (Burmeister, 1838) & \\
\hline & Myrsidea cucullaris (Nitzsch, 1818) & \\
\hline & Sturnidoecus sturni (Schrank, 1776) & \\
\hline \multicolumn{3}{|l|}{ Sylviidae } \\
\hline Sylvia nisoria (Bechstein, 1792) & Brueelia rosickyi Balát, 1955b & \\
\hline
\end{tabular}




\begin{tabular}{|c|c|c|}
\hline HOSTS & LICE & \\
\hline \multirow[t]{2}{*}{ Troglodytes troglodytes (Linnaeus, 1758) } & Penenirmus albiventris (Scopoli, 1763) & \\
\hline & Philopterus sp. & \\
\hline \multicolumn{3}{|l|}{ Turdidae } \\
\hline Turdus iliacus Linnaeus, 1758 & Menacanthus eurysternus (Burmeister, 1838)- & $\S * *$ \\
\hline \multirow[t]{4}{*}{ Turdus merula Linnaeus, 1758} & Guimaraesiella amsel (Eichler, 1951b) & \\
\hline & Philopterus turdi (Denny, 1842) & \\
\hline & Ricinus elongatus (Olfers, 1816) & \\
\hline & Turdinirmus merulensis (Denny, 1842) & \\
\hline \multirow[t]{2}{*}{ Turdus philomelos Brehm, 1831} & Menacanthus eurysternus (Burmeister, 1838) & \\
\hline & Philopterus turdi (Denny, 1842) & \\
\hline \multirow[t]{5}{*}{ Turdus pilaris Linnaeus, 1758} & Brueelia antimarginalis Eichler, 1951b & \\
\hline & Guimaraesiella marginata (Burmeister, 1838) & \\
\hline & Menacanthus eurysternus (Burmeister, 1838) & $\S$ \\
\hline & Philopterus bischoffi (Eichler, 1951b) & \\
\hline & Ricinus elongatus (Olfers, 1816) & \\
\hline \multirow[t]{4}{*}{ Turdus torquatus Linnaeus, 1758} & Brueelia intermedia (Nitzsch [in Giebel], 1866) & \\
\hline & Myrsidea thoracica (Giebel, 1874) & $\S * *$ \\
\hline & Philopterus sp. & \\
\hline & Ricinus elongatus (Olfers, 1816) & $\S$ \\
\hline \multirow[t]{3}{*}{ Turdus viscivorus Linnaeus, 1758} & Myrsidea thoracica (Giebel, 1874) & \# \\
\hline & Philopterus vernus (Złotorzycka, 1964) & \\
\hline & Ricinus elongatus (Olfers, 1816) & \\
\hline
\end{tabular}

\section{Pelecaniformes}

Ardeidae

Ardea alba Linnaeus, 1758

Ardea cinerea Linnaeus, 1758

Botaurus stellaris (Linnaeus, 1758)

Egretta garzetta (Linnaeus, 1766)

Ardeicola expallidus Blagoveshtchensky, 1940

Ardeicola ardeae (Linnaeus, 1758)

Ardeiphilus trochioxus (Burmeister, 1838)

Ardeicola stellaris (Denny, 1842)

Ciconiphilus decimfasciatus (Boisduval \& Lacordaire, \# 1835)

\section{Threskiornithidae}

Platalea leucorodia Linnaeus, 1758

Plegadis falcinellus (Linnaeus, 1766)

Ibidoecus plataleae (Denny, 1842)

Ardeicola rhaphidius (Nitzsch [in Giebel], 1866)

Ibidoecus bisignatus (Nitzsch [in Giebel], 1866)

\section{Piciformes}

\section{Picidae}

Dendrocopos leucotos (Bechstein, 1802)

Dendrocopos major (Linnaeus, 1758)

Brueelia straminea (Denny, 1842)

Penenirmus auritus (Scopoli, 1763)

Brueelia straminea (Denny, 1842)

Menacanthus pici (Denny, 1842) 


\begin{tabular}{ll}
\hline HOSTS & LICE \\
\hline Dendrocopos syriacus (Hemprich \& Ehrenberg, & Menacanthus sp. \\
1833) & Penenirmus auritus (Scopoli, 1763) \\
Dendrocoptes medius (Linnaeus, 1758) & Picicola superciliosa (Nitzsch [in Giebel], 1866) \\
Dryobates minor (Linnaeus, 1758) & Penenirmus auritus (Scopoli, 1763) \\
Dryocopus martius (Linnaeus, 1758) & Colpocephalum inaequale Burmeister, 1838 \\
& Penenirmus heteroscelis (Nitzsch, 1866) \\
Picus canus Gmelin, 1788 & Penenirmus pici (J.C. Fabricius, 1798) \\
Picus viridis Linnaeus, 1758 & Picicola candidus (Nitzsch, 1866) \\
& Menacanthus pici (Denny, 1842) \\
& Penenirmus pici (J.C. Fabricius, 1798) \\
& Picicola candidus (Nitzsch, 1866)
\end{tabular}

\section{Podicipediformes \\ Podicipedidae}

Podiceps auritus (Linnaeus, 1758)

Podiceps cristatus (Linnaeus, 1758)

Tachybaptus ruficollis (Pallas, 1764)

Aquanirmus colymbinus (Scopoli, 1763)

Pseudomenopon dolium (Rudow, 1869)

Aquanirmus podicepis (Denny, 1842)

Aquanirmus bahli Tandan, 1951

\section{Strigiformes}

\section{Strigidae}

Asio flammeus (Pontoppidan, 1763)

Asio otus (Linnaeus, 1758)

Athene noctua (Scopoli, 1769)

Bubo bubo (Linnaeus, 1758)

Bubo scandiacus (Linnaeus, 1758) — captive bird

Strix aluco Linnaeus, 1758

Strix uralensis Pallas, 1771

\section{Tytonidae}

Tyto alba (Scopoli, 1769)

\section{Suliformes}

\section{Phalacrocoracidae}

Phalacrocorax carbo (Linnaeus, 1758)

Eidmanniella pellucida (Rudow, 1869)

\#

Pectinopygus gyricornis (Denny, 1842)

\section{MAMMALIA}

Cetartiodactyla

Bovidae

Capra hircus Linnaeus, 1758-captive

Bovicola (Bovicola) caprae (Gurlt, 1843) 
TABLE 5. (Continued)

\begin{tabular}{ll}
\hline HOSTS & LICE \\
\hline $\begin{array}{l}\text { Rupicapra rupicapra (Linnaeus, 1758) } \\
\text { Cervidae } \\
\text { Capreolus capreolus (Linnaeus, 1758) }\end{array}$ & Bovicola (Bovicola) alpinus Kéler, 1942 \\
Carnivora & Damalinia (Cervicola) meyeri (Taschenberg, 1882) \\
Felidae & \\
$\quad$ Felis catus Linnaeus, 1758_captive & \\
Mustelidae & Felicola (Felicola) subrostratus (Burmeister, 1838) \\
Meles meles (Linnaeus, 1758) & \\
Mustela erminea Linnaeus, 1758 & Trichodectes melis (J.C. Fabricius, 1805) \\
Mustela nivalis Linnaeus, 1758 & Stachiella ermineae Hopkins, 1941 \\
Mustela putorius Linnaeus, 1758 & Stachiella mustelae (Schrank, 1803) \\
\hline
\end{tabular}

\section{Checklist of chewing lice}

\section{Order Phthiraptera Haeckel, 1896}

Suborder Amblycera Kellogg, 1896a

\section{Family Laemobothriidae Mjöberg, 1910}

\section{Laemobothrion (Eulaemobothrion) atrum (Nitzsch, 1818)}

Host: Fulica atra Linnaeus, 1758.

Ref.: Balát (1953) as Eulaemobothrion atrum (Nitzsch, 1818); Balát (1956, 1977).

Locations: Senec, 20 Nov. 1949; Bratislava_Rača, 16 Mar. 1952 (Balát 1956).

\section{Laemobothrion (Laemobothrion) tinnunculi (Linnaeus, 1758)}

Host: Falco tinnunculus Linnaeus, 1758.

Ref.: Balát (1953, 1956, 1977); this paper.

Locations: Trávnica, formerly Fíš, 2 May 1948 (Balát Coll., MMBC slide number 82); Bratislava, 3 Apr. 1951 (Balát 1956); Bratislava, 26 Jun. 2000 (Krištofík Coll., VETUNI).

\section{Laemobothrion sp.}

Host: Coracias garrulus Linnaeus, 1758.

Ref.: Balát (1953, 1956).

Location: Svätý Jur, Summer of 1948 (Balát Coll., MMBC slide numbers 43, 44).

Notes: There are only two lice, one on each slide, a third instar nymph and a teneral female. Since no species of Laemobothrion is known from any member of the family Coraciidae (Price et al. 2003: 311), we consider these lice as likely accidental stragglers on C. garrulus.

\section{Family Menoponidae Mjöberg, 1910}

\section{Actornithophilus gracilis (Piaget, 1880)}

Host: Vanellus vanellus (Linnaeus, 1758).

Ref.: Balát (1953) as Actornithophilus svobodae; Balát (1956, 1977) as Actornithophilus svobodai [sic]; Straka (1987) as Actornithophilus svobodai [sic].

Locations: Plavecké Podhradie, 29 Apr. 1951; Turňa nad Bodvou, 2 Nov. 1953 (Balát 1956); Kláštor pod Znievom, 2 and 20 Mar. 1978; Martin, 13 May 1982 (Straka Coll., AKMM). 
Amyrsidea perdicis (Denny, 1842)

Host: Phasianus colchicus Linnaeus, 1758.

Ref.: Balát (1956) as Amyrsidea sp. ?megalosoma; Balát (1977) as Amyrsidea megalosoma; Straka (1987) as Amyrsidea sp. ?megalosoma; Goldová et al. (2006).

Locations: Bratislava-Rača, 31 Dec. 1950 (Balát Coll., MMBC slide number 569); Trnava, 22 Nov. 1950; Bratislava, 3 Dec. 1950; Gabčíkovo, 21 Oct. 1954 (Balát 1956); Nové Zámky, 1 Apr. 1978 (Straka Coll., AKMM); Game Management Centre, Rozhanovce, 2000-2004 (Goldová et al. 2006).

Note: Straka (1987) recorded the date of collection as 15 Jan. 1982 but, according to Straka's notes on labels of all three available slides, the correct date is probably 1 Apr. 1978.

Amyrsidea phaeostoma (Nitzsch [in Giebel], 1866)

Host: Pavo cristatus Linnaeus, 1758 - captive bird.

Ref.: Straka (1987), this paper.

Location: Dražkovce, 14 Jan. 1985, Turčianská Štiavnička, 26 May 1979 (Straka Coll., AKMM).

Ardeiphilus trochioxus (Burmeister, 1838)

Host: Botaurus stellaris (Linnaeus, 1758).

Ref.: Balát (1953, 1956, 1977).

Location: Slovakia, May 1948 (Balát Coll., MMBC slide number 86).

\section{Austromenopon aegialitidis (Durrant, 1906)}

Host: Charadrius dubius Scopoli, 1786.

Ref.: Straka (1987) as Actornithophilus perrarus Blagoveshtchensky, 1948.

Location: Kláštor pod Znievom, 17 Apr. 1981 (Straka Coll., AKMM).

Notes: There is single slide with two females of A. aegialitidis labelled as Actornithophilus perrarus in Straka's collection. Straka (1987) reported these females as A. perrarus from Philomachus pugnax (Linnaeus, 1758), but host is given as "kulík riečny $=$ Charadrius dubius on the available slide. It is in accordance with host-louse association, despite louse genus was misidentified, because acording to Emerson (1972: 19) A. perrarus is a junior synonym of Actornithophilus ochraceus (Nitzsch, 1818), a parasite that is known from 20 species of plovers of the genera Charadrius and Pluvialis. (Price et al. 2003: 84). This is the first record of Austromenopon aegialitidis from Slovakia.

\section{Austromenopon crocatum (Nitzsch [in Giebel], 1866)}

Host: Numenius arquata (Linnaeus, 1758).

Ref.: Balát (1956, 1977).

Location: Senné, 16 Apr. 1950 (Balát Coll., MMBC slide number 458).

\section{Austromenopon decorosum Zlotorzycka, 1968}

Host: Tringa erythropus (Pallas, 1764).

Ref.: this paper.

Location: Senné, 20 Apr. 1950 (Balát Coll., MMBC slide number 456).

Note: This is the first record of Austromenopon decorosum from Slovakia.

\section{Austromenopon transversum (Denny, 1842)}

Host: Chroicocephalus ridibundus (Linnaeus, 1766).

Ref.: Balát (1953,1956) as Austromenopon ridibundus; Balát (1977) as Austromenopon transversum ridibundum; Straka (1987) as Austromenopon transversum ridibundum; this paper.

Locations: Bratislava_-Petržalka, 2 Nov. 1949 (Balát 1956); Jakubov-rybník, 20 Apr. 1997; Čunovo, 24 Apr. 1997; Gabčíkovo, 31 Jul. 1997 (Krištofík Coll., VETUNI); Sučany, 26 Nov. 1979; Kláštor pod Znievom 19 Jan. 1984 (Straka Coll., AKMM). 
Ciconiphilus decimfasciatus (Boisduval \& Lacordaire, 1835)

Host: Egretta garzetta (Linnaeus, 1758).

Ref.: this paper.

Locations: Kravany-Moča, 30 Jun. 2012 (Krištofík Coll., VETUNI).

Note: This is the first record of Ciconiphilus decimfasciatus from Slovakia.

\section{Ciconiphilus pectiniventris (Harrison, 1916)}

Host: Anser brachyrhynchus Baillon, 1834.

Ref.: Balát (1956) as Holomenopon sp.; this paper.

Location: Svätojurský Šúr, 24 Oct. 1953 (Balát Coll., MMBC slide numbers 44/e/10, 44/e/11).

Notes: Balát (1956) recorded Holomenopon sp. from this host. In the collection, there are two slides with Ciconiphilus pectiniventris from this host with the same data as in Balát (1956). We assume that Balát's (1956) record was based on these lice, and that he determined them incorrectly as Holomenopon. This is the first record of Ciconiphilus pectiniventris from Slovakia.

\section{Colpocephalum flavescens (Haan, 1829)}

Host: Haliaeetus albicilla (Linnaeus, 1758).

Ref.: Balát (1953) as Neocolpocephalum flavescens (Haan, 1829); Balát (1956, 1977).

Locations: Košice, Jan. 1949 (Balát Coll., MMBC slide number 310); Šal’a, 19 Mar. 1951; Šamorín, 9 Dec. 1952 (Balát 1956).

\section{Colpocephalum fregili Denny, 1842}

Host: Corvus corax Linnaeus, 1758.

Ref.: Straka (1987) as Colpocephalum subequale Burmeister, 1838.

Location: Ďanová, 1 Nov. 1977 (Straka Coll., AKMM).

\section{Colpocephalum fregili Denny, 1842}

Host: Corvus frugilegus Linnaeus, 1758.

Ref.: this paper.

Location: Kravany, 9 Jun. 1998 (Krištofík Coll., VETUNI).

Note: This is a new host-louse association for Slovakia.

\section{Colpocephalum impressum Rudow, 1866}

Host: Aquila chrysaetos (Linnaeus, 1758).

Ref.: Balát (1956, 1977); this paper.

Locations: Čachtice, 1 Feb. 1949 (Balát Coll., MMBC slide number 418); Volovské vrchy, Aug. 2020 (VETUNI).

Notes: Lice from Volovské vrchy were collected by ornithologist Milan Olekšák from a dead fledging bird. While handling this bird, he noticed that there were hundreds of lice, and collected some feathers with them. Later on, he felt itchy and then realised that dozens of lice were on his body (I. Literák \& M. Olekšák, pers. comm.).

\section{Colpocephalum impressum Rudow, 1866}

Host: Clanga pomarina (Brehm, 1831).

Ref.: this paper.

Location: Štrba, 2 Sep. 1938 (Pfleger Coll., SNMB).

Note: This is a new host-louse association for Slovakia.

\section{Colpocephalum inaequale Burmeister, 1838}

Host: Dryocopus martius (Linnaeus, 1758).

Ref.: Balát (1953, 1956, 1977); this paper.

Locations: Bratislava, 10 Oct. 1948 (Balát Coll., MMBC slide number 354); Bratislava, 7 Jun. 2010 (Krištofík Coll., VETUNI). 
Host: Buteo buteo (Linnaeus, 1758).

Ref.: this paper.

Locations: Farná, 13 Feb. 2001; Moravský Svätý Ján, 17 Dec. 2009; Závod, 11 Mar. 2010; Malé Leváre, 16 Mar. 2010 (Krištofík Coll., VETUNI).

Note: This is the first record of Colpocephalum nanum from Slovakia (see below).

\section{Colpocephalum nanum Piaget, 1890}

Host: Buteo lagopus (Pontoppidan, 1763).

Ref.: Balát (1956) as Colpocephalum sp. ?flavescens; Straka (1987) as Coplocephalum sp.; this paper.

Locations: Myslenice, 4 Feb. 1951; Bratislava, 12 Dec. 1952 (Balát 1956); Martin, 24 Jan. 1977 (Straka Coll., AKMM); Trnava, 15-28 Feb. 1939 (Pfleger Coll., SNMB).

Notes: Balát (1956) recorded Colpocephalum sp. from this host, noting that it may have been C. flavescens. Unfortunately, we cannot verify it, because there is no slide with Colpocephalum from Buteo lagopus in Balát's Collection. Considering that $C$. flavescens is a parasite of eagles of the genera Aquila and Haliaeetus (Price et al. 2003: 98), we believe that Balát's tentative identification is incorrect and that these lice are most likely $C$. nanum, a common parasite of $B$. lagopus (Price et al. 2003: 100). Furthermore, the Pfleger Collection includes slides with $C$. nanum from B. lagopus. Therefore, we can confirm that this is a valid host-louse association for Slovakia, as well as the first record of Colpocephalum nanum from Slovakia.

\section{Colpocephalum turbinatum Denny, 1842}

Host: Circus aeruginosus (Linnaeus, 1758).

Ref.: Balát (1953) as Neocolpocephalum bicinctum (Nitzsch [in Giebel] 1861); Balát $(1956,1977)$ as Colpocephalum bicinctum.

Location: Bratislava—Rača, 4 Sep. 1948 (Balát Coll., MMBC slide number 302).

\section{Colpocephalum zebra Burmeister, 1838}

Host: Ciconia ciconia (Linnaeus, 1758).

Ref.: Balát (1977); Straka (1987).

Locations: Veškovce, 5 Aug. 1959 (Balát Coll., MMBC slide number 1157); Martin, 17 Feb. 1977; Socovce, 30 Apr. 1980; Krpel'any, 3 Sep. 1982 (Straka Coll., AKMM).

\section{Colpocephalum sp.}

Host: Aquila heliaca Savigny, 1809.

Ref.: Balát (1956) as Colpocephalum sp. ?flavescens (Haan, 1829).

Location: Slovakia, 18 Sep. 1950 (Balát 1956).

Notes: Balát (1956) recorded Colpocephalum sp. ?flavescens from Aquila heliaca. Colpocephalum imperialis Martín-Mateo, 1981 was described from Aquila heliaca adalberti Brehm, 1861. However, as there is no specimen with the above data in the Balát Collection, we cannot confirm to which louse species this record refers.

\section{Colpocephalum sp.}

Host: Falco cherrug Gray, 1834.

Ref.: Balát (1956).

Location: Slovakia (Balát 1956).

Notes: Rak et al. (1975) reported Colpocephalum sp. from Falco cherrug in Iran. Further louse collections from this host are needed to confirm this host-louse association in Slovakia, as well as to identify the species.

\section{Colpocephalum sp.}

Host: Falco tinnunculus Linnaeus, 1758.

Ref.: Balát (1956) as Colpocephalum sp.

Location: Trávnica, formerly Fíš, May 1948 (Balát 1956).

Notes: According to Price et al. (2003: 102), Falco tinnunculus is parasitised by Colpocephalum subzerafae Ten- 
deiro, 1988. However, we cannot confirm this record to species level because there is no specimen of Colpocephalum from this host in the Balát Collection.

\section{Dennyus hirundinis (Linneaus, 1761)}

Host: Apus apus (Linnaeus, 1758).

Ref.: Straka (1987); this paper.

Locations: Martin, 7 Aug. 1979, 18 May 1996 (Straka Coll., AKMM); Bratislava, 15-19 Aug. 2002, 30 Jun. 2006, 27 Jul. 2007 and 18 Jul. 2010 (Krištofík Coll.,VETUNI).

\section{Eidmanniella pellucida (Rudow, 1869a)}

Host: Phalacrocorax carbo (Linnaeus, 1758).

Ref.: this paper.

Locations: Slovakia, May 1948 (Balát Coll., MMBC slide number 89).

Note: This is the first record of Eidmanniella pellucida from Slovakia.

\section{Gruimenopon longum (Giebel, 1874)}

Host: Grus grus (Linnaeus, 1758).

Ref.: Balát (1956, 1977).

Locations: Senné, Spring of 1955 (Balát Coll., MMBC slide numbers 1005-3x; 1006 - not present in the collection); Šamorín, 9 Dec. 1952 (Balát 1956).

\section{Heleonomus macilentus (Nitzsch [in Giebel], 1866)}

Host: Grus grus (Linnaeus, 1758).

Ref.: Balát (1977).

Location: Slovakia (Balát 1977).

Notes: Balát (1977) recorded Heleonomus macilentus from Slovakia without a host association. Considering that Grus grus is the only host for this louse species in Europe (Price et al. 2003: 110), we believe that Balát's (1977) record is also from this species of crane in Slovakia.

\section{Holomenopon clypeilargum Eichler, 1943a}

Host: Mergellus albellus (Linnaeus, 1758).

Ref.: Balát (1953, 1956) as Holomenopon sp; this paper.

Locations: Čilistov, 23 Mar. 1950 (Balát Coll., MMBC slide number 481); Senné, Spring of 1955 (Balát 1956).

Note: This is the first record of Holomenopon clypeilargum from Slovakia. Also, it is a new host-louse association worldwide (Price et al. 2003: 112).

\section{Holomenopon tadornae (Gervais, 1844)}

Host: Tadorna tadorna (Linnaeus, 1758).

Ref.: Balát (1956, 1977).

Location: Senné, no date (Balát 1956).

Kurodaia (Conciella) cryptostigmatia (Nitzsch [in Giebel], 1861)

Host: Athene noctua (Scopoli, 1769).

Ref.: Balát (1956, 1977).

Location: Plavecký Mikuláš, 14 Jan. 1951 (Balát Coll., MMBC slide number 582).

\section{Kurodaia (Conciella) cryptostigmatia (Nitzsch [in Giebel], 1861)}

Host: Strix uralensis Pallas, 1771.

Ref.: Hudec (1983) as Kurodaia sp.; this paper.

Location: Košice, 1 Dec. 1952 (Balát Coll., MMBC slide number 789).

Notes: Hudec (1983) recorded Kurodaia sp. from this host, but without giving a location. This record was probably made on the basis of slide 789 from the Balát Coll., because there is no other Kurodaia from this host in the collection. This is a new host-louse association worldwide (Price et al. 2003: 114). 


\section{Kurodaia (Conciella) subpachygaster (Piaget, 1880)}

Host: Tyto alba (Scopoli, 1769).

Ref.: Balát (1953, 1977).

Location: Gabčíkovo, 16 Sep. 1951 (Balát Coll., MMBC slide number 623).

Notes: Balát (1953) did not mention an exact location, and Balát (1977) recorded Kurodaia subpachygaster from Slovakia but without a host association. This record was probably made on the basis of slide 623 from the Balát Coll., because there is no other Kurodaia from this host in the collection.

\section{Kurodaia (Kurodaia) fulvofasciata (Piaget, 1880)}

Host: Buteo buteo (Linnaeus, 1758).

Ref.: this paper.

Location: Farná, 13 Feb. 2001 (Krištofík Coll., VETUNI).

Note: This is the first record of Kurodaia (Kurodaia) fulvofasciata from Slovakia.

\section{Kurodaia (Kurodaia) haliaeeti (Denny, 1842)}

Host: Pandion haliaetus (Linnaeus, 1758).

Ref.: Balát (1953, 1956, 1977).

Location: Bratislava—Devín, 16 May 1949 (Balát Coll., MMBC slide number 548).

\section{Machaerilaemus clayae (Balát, 1966)}

Host: Riparia riparia (Linnaeus, 1758).

Ref.: this paper.

Location: Gbelce, 28 Apr. 2008, 20 Apr. 2009 (VETUNI).

Note: This is the first record of Machaerilaemus clayae from Slovakia.

\section{Menacanthus agilis (Nitzsch, 1866)}

Host: Phylloscopus collybita (Vieillot, 1817).

Ref.: Hudec (1983) as Menacanthus phylloscopi (Nitzsch [in Giebel], 1866); this paper.

Location: Sklené Teplice, 18 Apr. 1953 (Balát Coll.,MMBC slide number 1461).

Notes: Hudec (1983) recorded Menacanthus phylloscopi from this host, but without giving a location. This record was probably made on the basis of slide 1461 from the Balát Coll., because there is no other Menacanthus agilis from this host from Slovakia in the collection. This is one of two first records of Menacanthus agilis from Slovakia (see below).

\section{Menacanthus agilis (Nitzsch, 1866)}

Host: Phylloscopus trochilus (Linnaeus, 1758).

Ref.: this paper.

Location: Nízké Tatry—Čertovica, 28 Jun. 1960 (Balát Coll., MMBC slide number 1412).

Note: This is one of two first records of Menacanthus agilis from Slovakia (see above).

\section{Menacanthus alaudae (Schrank, 1776)}

Host: Alauda arvensis Linnaeus, 1758.

Ref.: this paper.

Location: Malé Kosihy, 11 May 1997 (Krištofík Coll., VETUNI).

Note: This is a new host-louse association for Slovakia.

\section{Menacanthus alaudae (Schrank, 1776)}

Host: Carduelis carduelis (Linnaeus, 1758).

Ref.: Balát (1953, 1956, 1977) as Menacanthus carduelis (Denny, 1842); Straka (1987) as M. carduelis. Locations: Gabčíkovo, 22 Mar. 1954 (Balát 1956); Martin, 15 May 1982 (Straka Coll., AKMM). 
Host: Lanius collurio Linnaeus, 1758.

Ref.: this paper.

Locations: Vojka nad Dunajom, 27 Aug. 1997; Medved'ov, 17-18 Jul. 2000; Dvory nad Žitavou, 21 Jul. 2000 (Krištofík Coll., VETUNI).

Note: This is a new host-louse association for Slovakia.

\section{Menacanthus camelinus (Nitzsch [in Giebel], 1874)}

Host: Lanius excubitor Linnaeus, 1758.

Ref.: Balát (1977); Hudec (1983); Szczykutowicz et al. (2006) as Lanicanthus camelinus.

Locations: Slovakia (Balát 1977); NE Slovakia (Szczykutowicz et al. 2006).

Notes: Balát (1977) recorded Menacanthus camelinus from Slovakia without a host association. Balát (1977) also mentioned Menacanthus brevidentatus — now a junior synonym of $M$. camelinus (see below). Considering that the name M. brevidentatus was traditionaly used for lice associated with Lanius minor only, and that Lanius excubitor is the type host of $M$. camelinus, we can assume that the host of Balát's record is Lanius excubitor. Furthermore, Szczykutowicz et al. (2006) confirm this host-louse association for Slovakia.

\section{Menacanthus camelinus (Nitzsch [in Giebel], 1874)}

Host: Lanius minor Gmelin, 1788.

Ref.: Balát (1977) as Menacanthus brevidentatus Blagoveshtchensky, 1948.

Location: Zemplínska Široká-Rebrín, 6 Aug. 1959 (Balát Coll., MMBC slide numbers 1491, 1492-5x).

Notes: Balát (1977) recorded Menacanthus brevidentatus — now a junior synonym of M. camelinus (see Price 1977 : 210) - from Slovakia without a host association. Considering that Lanius minor is the type host of M. brevidentatus and that Balát's Coll. contains specimens from this host, we can safely assume that the host of Balát's record is Lanius minor.

\section{Menacanthus curuccae (Schrank, 1776)}

Host: Acrocephalus scirpaceus (Hermann, 1804).

Ref.: Sychra et al. (2008); Martinů et al. (2015); this paper.

Location: Gbelce, 14 Apr.-2 May 2008, 18 Apr.-1 May 2009, 18 Apr. 2016 (VETUNI).

Menacanthus curuccae (Schrank, 1776)

Host: Acrocephalus schoenobaenus (Linnaeus, 1758).

Ref.: Martinů et al. (2015); this paper.

Location: Gbelce, 15 Apr.-3 May 2008, 20 Apr.-1 May 2009 (VETUNI).

\section{Menacanthus eurysternus (Burmeister, 1838)}

Host: Coloeus monedula (Linnaeus, 1758).

Ref.: this paper.

Location: Pavlovce nad Uhom, 9 Apr. 1956 (Balát Coll., MMBC slide number 1186).

Note: This is a new host-louse association for Slovakia.

\section{Menacanthus eurysternus (Burmeister, 1838)}

Host: Corvus cornix Linnaeus, 1758.

Ref.: Balát (1956, 1977) as Menacanthus cornicis Blagoveshtchensky, 1948.

Location: Bratislava-Petržalka, 19 Feb. 1950 (Balát 1956).

Note: Although we could not find any specimens with the above data, we follow Price (1975: 619) regarding Menacanthus cornicis as a junior synonym of $M$. eurysternus.

Menacanthus eurysternus (Burmeister, 1838)

Host: Passer montanus (Linnaeus, 1758).

Ref.: Balát (1956) as Menacanthus annulatus (Giebel, 1874). 
Location: Gabčíkovo, 25 Mar. 1954 (Balát 1956).

Note: Although we could not find any specimens with the above data, we follow Price (1975: 617) regarding Menacanthus annulatus as a junior synonym of $M$. eurysternus.

\section{Menacanthus eurysternus (Burmeister, 1838)}

Host: Remiz pendulinus (Linnaeus, 1758).

Ref.: Krištofík (2000).

Location: Gbelce, 1 Aug. 1984; Devinské jazero, 26 Apr. 1994; Vysoká pri Morave, 18 May 1994; Dolný Štál, 31 Jul. 1994 (Krištofík 2000).

\section{Menacanthus eurysternus (Burmeister, 1838)}

Host: Sturnus vulgaris Linnaeus, 1758.

Ref.: Martinů et al. (2015); this paper.

Location: Gbelce, 13 Apr.-1 May 2008, 17 Apr. 2016, 2 Oct. 2019 (VETUNI).

\section{Menacanthus eurysternus (Burmeister, 1838)}

Host: Turdus iliacus Linnaeus, 1758.

Ref.: Straka (1987) as Myrsidea iliaca [sic].

Location: Turany-Trusalová, 30 Sep. 1982 (Straka Coll., AKMM).

Notes: Despite both available slides from this host, location and date are labelled as Menacanthus sp. Straka (1987) wrongly mentioned these lice as Myrsidea. Myrsidea iliaci Eichler, 1951a was described from one female from Turdus musicus (now Turdus iliacus). Clay (1966: 385) noted that: "This species is quite unrecognizable from the description ... the type is lost and ... nothing further can be said about this name." We assume that Straka (1987) misidentified this species only according to its host. This is a new host-louse association for Slovakia, and it is also a new host-louse association for Menacanthus eurysternus worldwide (Price et al. 2003: 132, Martinů et al. 2015).

\section{Menacanthus eurysternus (Burmeister, 1838)}

Host: Turdus philomelos Brehm, 1831.

Ref.: Balát (1977) as Menacanthus minusculus Blagoveshtchensky, 1940; this paper.

Location: Pavlovce nad Uhom, 9 Apr. 1956 (Balát Coll., MMBC slide number 1204).

Menacanthus eurysternus (Burmeister, 1838)

Host: Turdus pilaris Linnaeus, 1758.

Ref.: this paper.

Location: Štrbské pleso, 25 Jan. 1938 (Pfleger Coll., SNMB).

Note: This is a new host-louse association for Slovakia.

\section{Menacanthus fertilis (Nitzsch, 1866)}

Host: Upupa epops Linnaeus, 1758.

Ref.: Balát (1956, 1977).

Location: Bojnice, 18 Jun. 1953 (Balát 1956).

\section{Menacanthus gonophaeus (Burmeister, 1838)}

Host: Corvus corax Linnaeus, 1758.

Ref.: Balát (1977); Hudec (1983); this paper.

Locations: Slovakia (Balát 1977); Vel'ké Blahovo, 1 Jun. 2005 (Krištofík Coll., VETUNI).

Notes: Balát (1977) recorded Menacanthus gonophaeus from Slovakia without a host association. Considering that Corvus corax is the type host of M. gonophaeus, we assume that this is the host of Balát's record. Furthermore, we can confirm this host-louse association with our recent material. This is a new host-louse association for Slovakia. 
Menacanthus gonophaeus (Burmeister, 1838)

Host: Corvus frugilegus Linnaeus, 1758.

Ref.: Balát (1977); Straka (1987) as Menacanthus laticeps Blagoveshtchensky, 1948; this paper.

Locations: Stará Bystrica, 5 Jan. 1982 (Straka Coll., AKMM); Zlatná na Ostrove, 20 Jan. 2001 (Krištofík Coll., VETUNI).

Notes: Balát (1977) recorded Menacanthus laticeps-now a junior synonym of M. gonophaeus (see Price 1977 : 208) - from Slovakia without a host association. However, based on other associations of Menacanthus gonophaeus with Corvus frugilegus, we can assume that this is the host of Balát's record. Furthermore, we confirm this host-louse association as recorded by Straka (1987) and from our recent material. Straka (1987) recorded the location as "Krpel'any", but it is most likely an error, because in Straka's note on label of an available slide the location is given as "Stará Bystrica".

\section{Menacanthus gonophaeus (Burmeister, 1838)}

Host: Coloeus monedula (Linnaeus, 1758).

Ref.: Balát (1956, 1977) as Menacanthus monedulae Blagoveshtchensky, 1951.

Location: Bratislava, 10 Oct. 1948 (Balát Coll., MMBC slide number 352).

Notes: Balát (1977) recorded Menacanthus monedulae - now a junior synonym of M. gonophaeus (see Price 1977: 208) - from Slovakia without a host association. However, based on other Menacanthus associations with Coloeus monedula, we can assume that this is the host of Balát's record.

Menacanthus chrysophaeus (Kellogg, 1896b)

Host: Emberiza schoeniclus (Linnaeus, 1758).

Ref.: Martinu et al. (2015); this paper.

Location: Gbelce, 12-15 Apr. 2008, 19 Apr. 2009 (VETUNI).

Menacanthus merisuoi Eichler, 1953c

Host: Nucifraga caryocatactes (Linnaeus, 1758).

Ref.: this paper.

Locations: Martin, 3 Dec. 1981 (Straka Coll., AKMM).

Note: This is the first record of Menacanthus merisuoi from Slovakia.

\section{Menacanthus obrteli Balát, 1981b}

Host: Locustella luscinioides (Savi, 1824).

Ref.: Martinů et al. (2015); this paper.

Location: Gbelce, 12 Apr.-1 May 2008, 19 Apr.-1 May 2009, 17 Apr. 2016 (VETUNI).

\section{Menacanthus pici (Denny, 1842)}

Host: Dendrocopos major (Linnaeus, 1758).

Ref.: this paper.

Location: Malženice, 11 Nov. 2006 (Krištofík Coll., VETUNI).

Note: This is a new host-louse association for Slovakia.

\section{Menacanthus pici (Denny, 1842)}

Host: Picus viridis Linnaeus, 1758.

Ref.: Balát (1953, 1956, 1977).

Locations: Bratislava, 10 Oct. 1948 (Balát Coll., MMBC slide number 353); Bratislava, 20 Dec. 1951; Žiar nad Hronom, formerly Svätý Kríž nad Hronom, 10 Oct. 1953 (Balát 1956).

\section{Menacanthus pusillus (Nitzsch, 1866)}

Host: Anthus spinoletta (Linnaeus, 1758).

Ref.: Balát (1955a, 1956) as Menacanthus sp.

Locations: Beliansk Tatry—Bujačí vrch, 29 Apr. 1952; Belianské Tatry-šafránová louka/pašienok pod Bujačím vrchom, 3 May 1952 (Balát Coll., MMBC slide numbers 642, 703). 
Host: Motacilla cinerea Tunstall, 1771.

Ref.: Balát (1956) as Menacanthus sp.

Locations: Košice, 5 Nov. 1953; Sklené Teplice, 14 Apr. 1953 (Balát Coll., MMBC slide numbers 451-3x, 749, 1489).

\section{Menacanthus pusillus (Nitzsch, 1866)}

Host: Motacilla flava Linnaeus, 1758.

Ref.: this paper.

Location: Vel'ké Kapušany, 17 Apr. 1959, 18 Apr. 1959 (Balát Coll., MMBC slide numbers 1175, 1220-5x, 1221$2 \mathrm{x}, 1483,1484)$.

Note: This is a new host-louse association for Slovakia.

Menacanthus sinuatus (Burmeister, 1838)

Host: Cyanistes caeruleus (Linnaeus, 1758).

Ref.: Balát (1956) as Menacanthus sp.

Locations: Sklené Teplice, 14-18 Apr. 1953; Šaca, 4 Nov. 1953; Gabčíkovo, 16 Mar. 1954 (Balát 1956).

Note: Although we could not find any specimens with the above data, we follow Price (1977: 217) who recorded specimens of Menacanthus sinuatus from C. caeruleus (as Parus caeruleus).

\section{Menacanthus sinuatus (Burmeister, 1838)}

Host: Parus major Linnaeus, 1758.

Ref.: Balát (1956, 1977); Straka (1987) as Menacanthus sinuatus sinuatus; Martinů et al. (2015); this paper.

Locations: Gabčíkovo, 18 Mar. 1954 (Balát Coll., MMBC slide number 1196); Sklené Teplice, 14-18 Apr. 1953, 6-7 Oct. 1953; Žiar nad Hronom, formerly Svätý Kríž nad Hronom-dolina Kl'ak, 9 Oct. 1953; Gabčíkovo, 15-16 Mar. 1954 (Balát 1956); Vrícko, 15 Nov. 1982; (Straka Coll., AKMM); Bratislava—Mlynská Dolina, 1 Mar. 1999 (Krištofík Coll., VETUNI); Gbelce, 17 Apr. 2008 (VETUNI).

Note: Straka (1987) recorded data on collections of Menacanthus sinuatus from Parus major and Periparus ater, but without mentioning which locality referred to each host species. Here we mention locations separately according to Straka's notes on relevant slides.

Menacanthus sinuatus (Burmeister, 1838)

Host: Periparus ater (Linnaeus, 1758).

Ref.: Balát (1956) as Menacanthus sp. ?sinuatus; Balát (1977) as Menacanthus bussei Złotorzycka, 1965; Straka (1987) as Menacanthus sinuatus bussei.

Locations: Ihráč, 18 Apr. 1953 (Balát Coll., MMBC slide number 748); Klášor pod Znievom, 11 Mar. 1985 (Straka Coll., AKMM).

Notes: Balát (1956) recorded the date of collection as (18 Apr. 1954) but, according to Balát's notes, the correct date is 18 Apr. 1953. Straka (1987) recorded data on collections of Menacanthus sinuatus from Parus major and Periparus ater, but without mentioning which locality referred to each host species. Here we mention locations separately according to Straka's notes on relevant slides.

\section{Menacanthus sinuatus (Burmeister, 1838)}

Host: Poecile palustris (Linnaeus, 1758).

Ref.: Balát (1956, 1977).

Locations: Sklené Teplice, 21 Apr. \& 6 Oct. 1953; Jablonov, 3 Nov. 1953; Košice, 5 Nov. 1953 (Balát 1956).

\section{Menacanthus stramineus (Nitzsch, 1818)}

Host: Gallus gallus (Linnaeus, 1758)_captive bird.

Ref.: Straka (1987) as Menacanthus sp.

Location: Kláštor pod Znievom, 25 Apr. 1977 (Straka Coll., AKMM).

Note: This is the first record of Menacanthus stramineus from Slovakia. 


\section{Menacanthus sp.}

Host: Dendrocopos syriacus (Hemprich \& Ehrenberg, 1833).

Ref.: Balát (1956) as Menacanthus sp. ?dryobates (Eichler, 1953c).

Location: Gabčíkovo, 21 Oct. 1954 (Balát 1956).

Notes: Balát (1956) recorded Menacanthus cf. dryobates from Dendrocopos syriacus, but this louse species is a junior synonym of Menacanthus pici and there has not been any further record of Menacanthus from D. syriacus (see Price et al. 2003: 366). This may be a new host-louse association for M. pici, a species that occurs on other 26 species of woodpeckers (Price et al. 2003: 124), or it may be and accidental straggler. Unfortunately, there are no specimens of Menacanthus from D. syriacus in the Balát Collection to confirm this record at the species level.

\section{Menacanthus sp.}

Host: Panurus biarmicus (Linnaeus, 1758).

Ref.: this paper.

Location: Gbelce, 19-24 Apr. 2009, 19 Apr. 2016, 8-11 Jul. \& 1 Oct. 2019 (VETUNI).

Notes: Menacanthus brelihi was described from Panurus biarmicus by Balát (1981b) on the basis of material from the Czech Republic. Subsequently, it was synonymised under M. eurysternus by Krištofík (2000). Our examination of the type specimens of M. brelihi deposited at MMBC and other material from Gbelce confirms that lice from Panurus biarmicus belong to a valid species different from M. eurysternus (Ošlejšková \& Sychra, unpublished data). However, we list this record as Menacanthus sp. until the resurrection of M. brelihi is formalised. This is a new host-louse association for Slovakia.

\section{Menopon gallinae (Linnaeus, 1758)}

Host: Gallus gallus (Linnaeus, 1758) — captive bird.

Ref.: this paper.

Location: Kláštor pod Znievom, 25 Apr. 1977 (Straka Coll., AKMM).

Note: Although the occurrence of this louse species if probably well known among veterinarians and hen breeders, this is the first record of Menopon gallinae from Slovakia. Specimens in the Straka's collection are mounted partially together with Menacanthus stramineus and Goniocotes gallinae on the same slides reported by Straka (1987) as Menacanthus sp.

\section{Menopon pallens Clay, 1949b}

Host: Perdix perdix (Linnaeus, 1758).

Ref.: Balát (1956, 1977); Straka (1987).

Locations: Trnava, 7 Oct. 1951 (Balát Coll., MMBC slide number 618); Martin, 20 Jan. 1977 (Straka Coll., AKMM).

\section{Meromenopon meropis Clay \& Meinertzhagen, 1941}

Host: Merops apiaster Linnaeus, 1758.

Ref.: Balát (1953, 1956, 1977); Krištofík et al. (1996); this paper.

Locations: Radošovce_-Vieska, 25 Jul. 1949; Sered', 4 Sep. 1948 (Balát Coll., MMBC—slide numbers 213, 453, SNMB slide number 452); Jurský Chlm, Malá nad Hronom, Pavlová, Sikenička, May-Jul. 1995 for all locations (Krištofík et al. 1996); Virt, 19 Jul. 1998 (Krištofík Coll., VETUNI).

\section{Myrsidea anathorax (Nitzsch, 1866)}

Host: Coloeus monedula (Linnaeus, 1758).

Ref.: this paper.

Locations: Bratislava, 10 Oct. 1948; Palín, 7 Apr. 1956; Pavlovce nad Uhom, 15 Apr. 1959 (Balát Coll., MMBC slide numbers $352,1166,1188$ ).

Note: This is the first record of Myrsidea anathorax from Slovakia. 


\section{Myrsidea cornicis (De Geer, 1778)}

Host: Corvus cornix Linnaeus, 1758.

Ref.: Balát (1953, 1956, 1977); Straka (1987).

Locations: Bratislava_Petržalka, 19 Feb. 1950; Žihárec/Zsigárd, 17 Sep. 1953 (Balát Coll., MMBC slide number 526, B287); Zlaté Moravce, 12 Oct. 1953; Tatranská Lomnica, 8 Jun. 1955 (Balát 1956); Martin, 27 Jan. 1977 (Straka Coll., AKMM).

\section{Myrsidea cucullaris (Nitzsch, 1818)}

Host: Sturnus vulgaris Linnaeus, 1758.

Ref.: Balát (1956, 1977); this paper.

Locations: Banská Štiavnica—Počúvadla, 23 Apr. 1953 (Balát Coll., MMBC slide number 1125); Gbelce, 2 Oct. 2019 (VETUNI).

\section{Myrsidea franciscoloi Conci, 1942}

Host: Cinclus cinclus (Linnaeus, 1758).

Ref.: Balát (1953, 1956, 1977).

Locations: Žarnovica, 7 Jan. 1952; Sklené Teplice, 11 Oct. 1953 (Balát 1956).

\section{Myrsidea isostoma (Nitzsch, 1866)}

Host: Corvus frugilegus Linnaeus, 1758.

Ref.: this paper.

Location: Kravany, 9 Jun. 1998 (Krištofík Coll., VETUNI).

Note: This is the first record of Myrsidea isostoma from Slovakia.

\section{Myrsidea latifrons (Carriker [in Carriker \& Shull], 1910)}

Host: Riparia riparia (Linnaeus, 1758).

Ref.: this paper.

Location: Gbelce, 28 Apr. 2008, 27 Apr. 2009 (VETUNI).

Note: This is the first record of Myrsidea latifrons from Slovakia.

Myrsidea picae (Linnaeus, 1758)

Host: Pica pica (Linnaeus, 1758).

Ref.: Balát (1956, 1977); Straka (1987).

Locations: Vel'ké Topol'níky, 14 Feb. 1951; Šaca, 1 Nov. 1953 (Balát 1956); Blatnica, 31 Jan. 1977 (Straka Coll., AKMM).

\section{Myrsidea quadrifasciata quadrifasciata (Piaget, 1880)}

Host: Passer montanus (Linnaeus, 1758).

Ref.: Sychra et al. (2021).

Locations: Gabčíkovo, 22 Jul. 1953 (Balát Coll., MMBC slide number 1380); Gbelce, (4751’N 1830’E), 10 Jul. 2019 (VETUNI).

Note: Sychra et al. (2021) have recognised several subspecies of Myrsidea quadrifasciata, with the population from Passer montanus belonging to the nominate subspecies.

\section{Myrsidea rustica (Giebel, 1874)}

Host: Hirundo rustica Linnaeus, 1758.

Ref.: this paper.

Location: Gbelce, 17 Apr.-2 May 2008, 19-30 Apr. 2009 (VETUNI).

Note: This is the first record of Myrsidea rustica from Slovakia.

Myrsidea thoracica (Giebel, 1874)

Host: Turdus torquatus Linnaeus, 1758. 
Ref.: Hudec (1983) as Myrsidea sp.; this paper.

Location: Nízke Tatry-Čertovica, 29 Jun. 1960 (Balát Coll., MMBC slide number 1211).

Notes: Hudec (1983) recorded Myrsidea sp. from this host, but without giving a location. This record was probably made on the basis of slide 1211 from the Balát Coll., because there is no other Myrsidea from this host in the collection This is a first record of Myrsidea thoracica from Slovakia, and it is also a new host-louse association for Myrsidea thoracica worldwide (Price et al. 2003: 132).

\section{Myrsidea thoracica (Giebel, 1874)}

Host: Turdus viscivorus Linnaeus, 1758.

Ref.: Hudec (1983) as Myrsidea sp.; this paper.

Location: Nízke Tatry —Čertovica, 29 Jun. 1960 (Balát Coll., MMBC slide number 1210-2x).

Notes: Hudec (1983) recorded Myrsidea sp. from this host, but without giving a location. This record was probably made on the basis of slide 1210-2x from the Balát Coll., because there is no other Myrsidea from this host in the collection. Together with the above, this is a new record of Myrsidea thoracica from Slovakia.

\section{Nosopon lucidum (Rudow, 1869a)}

Host: Falco tinnunculus Linnaeus, 1758.

Ref.: this paper.

Location: Královičove Kračany, 26 May 2003 (Krištofík Coll., VETUNI).

Note: This is the first record of Nosopon lucidum from Slovakia.

\section{Pseudomenopon dolium (Rudow, 1869a)}

Host: Podiceps cristatus (Linnaeus, 1758).

Ref.: Balát (1953) as Pseudomenopon tridens (Burmeister, 1838); Balát (1956, 1977).

Location: Gabčíkovo, 22 Jul. 1953 (Balát 1956).

Note: Although we could not find any specimens with the above data, we follow Balát $(1956,1977)$ who recorded Pseudomenopon dolium from Podiceps cristatus.

\section{Pseudomenopon pilosum (Scopoli, 1763)}

Host: Fulica atra Linnaeus, 1758.

Ref.: this paper.

Location: Bratislava-Kopáč, 19 Feb. 1997 (Krištofík Coll., VETUNI).

Note: This is a new host-louse association for Slovakia.

\section{Pseudomenopon pilosum (Scopoli, 1763)}

Host: Gallinula chloropus (Linnaeus, 1758).

Ref.: Balát (1956) as Pseudomenopon tridens (Burmeister, 1838), this paper.

Locations: Cífer, 2 May 1951 (Balát Coll., MMBC slide number 796-6x); Komárno, 1953 (Balát 1956); Martin, 6 Apr. 1982 (Straka Coll., AKMM).

\section{Trinoton anserinum (J.C. Fabricius, 1805)}

Host: Anser brachyrhynchus Baillon, 1834.

Ref.: Balát (1956) as Trinoton sp. ?anserinum; Balát (1977); Straka (1987).

Locations: Svätojurský Šúr, 24 Oct. 1953 (Balát 1956); Lipovec, 22 Dec. 1981 (Straka Coll., AKMM).

Notes: Balát (1956) recorded Trinoton cf. anserinum from Anser brachyrhynchus, but there is no available specimen of Trinoton from this host in the Balát Collection; therefore, we can not confirm his record to species level. However, Straka (1987) confirmed that Anser brachyrhynchus is parasitised by T. anserinum, a host-louse association that was not listed by Price et al. (2003: 138).

Trinoton anserinum (J.C. Fabricius, 1805)

Host: Anser fabalis (Latham, 1787).

Ref.: Balát (1956) as Trinoton sp. ?anserinum; Balát (1977). 
Location: Čilistov, 5 Nov. 1949 (Balát Coll., MMBC slide number 1362).

Notes: We confirm that Balát's records refer to Trinoton anserinum. This host-louse association was not listed by Price et al. (2003: 138).

Trinoton anserinum (J.C. Fabricius, 1805)

Host: Cygnus olor (Gmelin, 1789).

Ref.: Straka (1987) as Trinoton cygni Eichler, 1943b.

Location: Ďanová, 1 Mar. 1977 (Straka Coll., AKMM)

Trinoton querquedulae (Linnaeus, 1758)

Host: Anas acuta Linnaeus, 1758.

Ref.: Balát (1956) as Trinoton sp.

Location: Senné, Jun. 1951 (Balát Coll., MMBC slide number 598).

Note: We confirm that Balát's record refers to Trinoton querquedulae.

Trinoton querquedulae (Linnaeus, 1758)

Host: Anas crecca Linnaeus, 1758.

Ref.: Balát (1956) as Trinoton sp.; Straka (1987).

Locations: Bratislava, 20 Nov. 1951 (Balát 1956); Martin, 18 Nov. 1982 (Straka Coll., AKMM).

Note: Straka (1987) recorded data about collections of Trinoton querquedulae from Anas crecca and Spatula querquedula, but without mentioning which locality referred to each host species. Here we mention locations separately according to Straka's notes on relevant slides.

Trinoton querquedulae (Linnaeus, 1758)

Host: Anas platyrhynchos Linnaeus, 1758.

Ref.: Balát (1953); Balát (1956) as Trinoton sp.; Straka (1987) as Trinoton sp., this paper.

Locations: Bratislava, 28 Dec. 1948; Sládkovičovo, 16 Oct. 1951 (Balát 1956); Martin, 19 Nov. 1976, 20 Oct. \& 3 Dec. 1977, Kláštor pod Znievom, 1. Nov. 1978 (Straka Coll., AKMM)

Trinoton querquedulae (Linnaeus, 1758)

Host: Aythya ferina (Linnaeus, 1758).

Ref.: this paper.

Location: Jakubovské rybníky, no data (Krištofík Coll., VETUNI).

Note: This is a new host-louse association for Slovakia.

Trinoton querquedulae (Linnaeus, 1758)

Host: Aythya fuligula (Linnaeus, 1758).

Ref.: this paper.

Location: Jakubov, 13 Jul. 1997 (Krištofík Coll., VETUNI).

Note: This is a new host-louse association for Slovakia.

Trinoton querquedulae (Linnaeus, 1758)

Host: Mergellus albellus (Linnaeus, 1758).

Ref.: Balát $(1953,1956,1977)$ as Trinoton lituratum Burmeister, 1838.

Locations: Čilistov, 23 Mar. 1950; Šal'a, 26 Jan. 1951 (Balát Coll., MMBC slide numbers 481, 584); Gabčíkovo, 17-24 Mar. 1954 (Balát 1956).

Note: Trinoton lituratum is a junior synonym of Trinoton querquedulae (see Price et al. 2003: 138).

Trinoton querquedulae (Linnaeus, 1758)

Host: Spatula querquedula (Linnaeus, 1758).

Ref.: Straka (1987).

Location: Dražkovce, 20 Apr. 1981 (Straka Coll., AKMM). 
Note: Straka (1987) recorded data about collections of Trinoton querquedulae from Anas crecca and Spatula querquedula, but without mentioning which locality referred to each host species. Here we mention locations separately according to Straka's notes on relevant slides.

\section{Family Ricinidae Neumann, 1890}

\section{Ricinus dolichocephalus (Scopoli, 1763)}

Host: Oriolus oriolus (Linnaeus, 1758).

Ref.: Balát (1956, 1977).

Location: Járok u Nitry, 16 Jun. 1953 (Balát Coll., MMBC slide number 1055).

\section{Ricinus elongatus (Olfers, 1816)}

Host: Turdus merula Linnaeus, 1758.

Ref.: Balát (1952, 1953); Balát (1956) as Ricinus elongatus ernstlangi Eichler, 1941a; Balát (1977); Straka (1987) as $R$. ernstlangi.

Locations: Čilistov, 26 Mar. 1950 (Balát Coll., MMBC slide number 449—not present in the collection); Kláštor pod Znievom, 2 Mar. 1978 (Straka Coll., AKMM).

\section{Ricinus elongatus (Olfers, 1816)}

Host: Turdus pilaris Linnaeus, 1758.

Ref.: Balát (1952, 1956); Straka (1987); Bush et al. (2018).

Locations: Štrbské pleso, 25 Jan. 1938 (Balát 1952, 1956); Vrícko, 17 Jan. 1979 (Straka Coll., AKMM); Tatranská Javorina, Jun.-Jul. 2015 (Bush et al. 2018).

Note: Straka (1987) recorded the location and date as "Ležiachov, 17 Jan. 1974, but it is most likely an error, because in Straka's note on label of an available slide the location and date are given as "Vrícko, 17 Jan. 1979.

\section{Ricinus elongatus (Olfers, 1816)}

Host: Turdus torquatus Linnaeus, 1758.

Ref.: this paper.

Locations: Martin, 5 May 1982 (Straka Coll., AKMM).

Note: This is a new host-louse association for Slovakia.

\section{Ricinus elongatus (Olfers, 1816)}

Host: Turdus viscivorus Linnaeus, 1758.

Ref.: Balát (1956).

Locations: Žiar nad Hronom, formerly Svätý Kríž nad Hronom—dolina Kl’ak, 16 Apr. 1953 (Balát Coll., MMBC slide number 739-4x).

Note: Balát (1956) recorded the location as Sklené Teplice, but it is most likely an error, because in Balát's notes the location is given as "dolina Kl'ak".

\section{Ricinus fringillae De Geer, 1778}

Host: Anthus spinoletta (Linnaeus, 1758).

Ref.: Balát (1955a, 1956) as Ricinus japonicus Uchida, 1915.

Locations: Belianské Tatry—šafránová louka/pašienok pod Bujačím vrchom, 2-3 May 1952; Nízké Tatry—hotel Srdiečko, 30 Jun. 1960; Vysoké Tatry—Skalnaté pleso,15 Jun. 1955 (Balát Coll., MMBC slide numbers 655, 699, 701, 1163); Beliansk Tatry—šafránov louka/pašienok pod Bujačím vrchom, 29 Apr. 1952 (Balát 1955a).

Note: We identified the material in the Balát Collection as Ricinus fringillae, the senior synonym of $R$. japonicus.

\section{Ricinus fringillae De Geer, 1778}

Host: Emberiza citrinella Linnaeus, 1758.

Ref.: Balát (1953, 1956); Straka (1987). 
Locations: Gabčíkovo, Apr. 1954; Járok u Nitry, 16 Mar. 1953; Krišovská Liesková-Krížany, 14 Apr. 1959; Plášt'ovce, 27 Apr. 1956; Banská Štiavnica—Počúvadla, 23 Apr. 1953; Senné, 16 Apr. 1950; Sklené Teplice, 14 Apr. 1953; Slovakia, 21 Jun. 1953; Svätojurský Šúr, 13 Feb. 1951; Veškovce, 16 Apr. 1959 (Balát Coll., MMBC slide numbers 507, 644, 1101, 1329, 1330, 1332, 1333, 1334, 1336, 1337); Banská Štiavnica—Počúvadla, 21 Jun. 1953; Podunajsk Biskupice, 21 Jul. 1953; Gabčíkovo, 15 Mar., 24 Mar. \& 6 May 1954; Járok u Nitry, 16 Jun. 1953 (Balát 1956); Kláštor pod Znievom, 9 Feb. \& 13 Mar. 1981; (Straka Coll., AKMM).

Notes: Balát (1956) recorded a location and date as Járok u Nitry (16 Jun. 1953)but, according to Balát's notes there are two dates 16 Mar. 1953 and 16 Jun. 1953. Straka (1987) recorded data on collections of Ricinus fringillae from Emberiza citrinella and E. schoeniclus, but without mentioning which locality referred to each host species. Here we mention locations separately according to Straka's notes on relevant slides.

\section{Ricinus fringillae De Geer, 1778}

Host: Emberiza schoeniclus (Linnaeus, 1758).

Ref.: Balát (1952, 1956); Straka (1987); this paper.

Locations: Senné, 16 Apr. 1950 (Balát Coll., MMBC slide numbers 504, 573a; SNMB slide number 573b); ŠPR Kláštorské lúky pri Kláštore pod Znievom, 25 Apr. 1978, 10 May 1982 (Straka Coll., AKMM); Gbelce, 23 Apr. 2008 (VETUNI).

Note: Straka (1987) recorded data on collections of Ricinus fringillae from Emberiza citrinella and E. schoeniclus, but without mentioning which locality referred to each host species. Here we mention locations separately according to Straka's notes on relevant slides.

\section{Ricinus fringillae De Geer, 1778}

Host: Fringilla coelebs Linnaeus, 1758.

Ref.: Balát (1952, 1953, 1956) as Ricinus irascens (Burmeister, 1838); Balát (1977).

Locations: Sklené Teplice, 20 Apr. 1953 (Balát Coll., MMBC slide number 1056); Podunajsk Biskupice, 16 Apr. 1951; Banská Štiavnica—Počúvadla, 23 Apr. 1953; Gabčíkovo, 23-24 Mar. 1954 (Balát 1952, 1956).

Note: We identified the material in the Balát Collection as Ricinus fringillae, the senior synonym of $R$. irascens.

\section{Ricinus fringillae De Geer, 1778}

Host: Prunella collaris (Scopoli, 1769).

Ref.: Balát (1955a, 1956, 1977); Janiga \& Kubašková (2000) as Ricinus subpallidus Blagoveshtchensky, 1951.

Locations: Vysoké Tatry - Skalnaté pleso, 15 Jun. 1955 (Balát Coll., MMBC slide number 697); Vysok Tatry, Nízké Tatry, 1988-1999 (Janiga \& Kubašková 2000).

Note: Ricinus subpallidus is a junior synonym of R. fringillae (see Price et al. 2003: 251).

\section{Ricinus rubeculae (Schrank, 1776)}

Host: Erithacus rubecula (Linnaeus, 1758).

Ref.: Balát (1952, 1953, 1956, 1977); Straka (1987).

Locations: Čilistov, 26 Mar. 1950; Žiar nad Hronom, formerly Svätý Kríž nad Hronom—dolina Klak, 16 Apr. 1953; Holíč, 27 Mar. 1948; Podunajsk Biskupice, 20 Jul. 1953; Sklené Teplice, 20 Apr. 1953, 20 Jun. 1953, 24 Apr. 1953 (Balát Coll., MMBC slide numbers 13-2x, 14, 448, 1048, 1071, 1109-2x, 1110, 1111); Podunajsk Biskupice, 16 Apr. 1951; Sklené Teplice, 18 Apr. \& 6 Oct. 1953 (Balát 1952, 1956); Kláštor pod Znievom, 2-3 Apr. 1979 (Straka Coll., AKMM).

\section{Ricinus serratus (Durrant, 1906)}

Host: Galerida cristata (Linnaeus, 1758).

Ref.: this paper.

Location: Krišovská Liesková—Krížany, 15 Apr. 1959 (Balát Coll., MMBC slide number 1338).

Note: This is the first record of Ricinus serratus from Slovakia.

\section{Ricinus sp.}

Host: Oenanthe oenanthe (Linnaeus, 1758). 
Ref.: Balát (1955a, 1956).

Location: Vysoké Tatry - Skalnaté pleso, 15 Jun. 1955 (Balát 1955a, 1956).

Notes: This is the only published record of Ricinus from Oenanthe oenanthe. However, considering that we could neither find material in the Balát Collection, nor any other specimen from $O$. oenanthe, we are not able to confirm if Ricinus is a natural and regular parasite of this host.

\section{Suborder Ischnocera Kellogg, 1896a}

\section{Family Philopteridae Burmeister, 1838}

\section{Acronirmus gracilis (Burmeister, 1838)}

Host: Delichon urbicum (Linnaeus, 1758).

Ref.: Balát (1953, 1955b, 1956, 1977) as Brueelia gracilis; Straka (1987) as Brueelia gracilis; this paper.

Locations: Bojnice, 23 Apr. 1954 (Balát Coll., MMBC slide number 684); Nový Smokovec, 18 Apr. 1955 (Balát 1955a); Martin, 11 Sep. 1980 (Straka Coll., AKMM); Malacky-Vinohrádok, 12 Feb. 2002 (Krištofík Coll., VETUNI).

Note: We follow Gustafsson \& Bush (2017: 60) in placing this louse species in the genus Acronirmus.

\section{Acronirmus gracilis (Burmeister, 1838)}

Host: Hirundo rustica Linnaeus, 1758.

Ref.: this paper.

Location: Gbelce, 19 Apr.-2 May 2008, 19 Apr.-1 May 2009 (VETUNI).

Note: This is a new host-louse association for Slovakia.

\section{Alcedoffula alcedinis (Denny, 1842)}

Host: Alcedo atthis (Linnaeus, 1758).

Ref.: Balát (1953, 1956, 1977).

Locations: Radošovce — Vieska (Balát, 1956 recorded only Vieska, district Senica), 10 Jul. 1949; Sklené Teplice, 11 Oct. 1953; Humenné, 25 Jul. 1954 (Balát 1956).

Anaticola anseris (Linnaeus, 1758)

Host: Anser albifrons (Scopoli, 1769).

Ref.: Balát (1956, 1977) as Anaticola serratus (Nitzsch [in Giebel], 1866]; Straka (1987) as A. serratus.

Locations: Šurany, 11 Nov. 1950 (Balát 1956); Sučany, 5 and 10 Oct. 1977 (Straka Coll., AKMM)

\section{Anaticola anseris (Linnaeus, 1758)}

Host: Anser brachyrhynchus Baillon, 1834.

Ref.: Balát (1956, 1977) as Anaticola sp. ?anseris; Straka (1987).

Locations: Svätojurský Šúr, 24 Oct. 1953 (Balát 1956); Lipovec, 22 Dec. 1981 (Straka Coll., AKMM).

\section{Anaticola anseris (Linnaeus, 1758)}

Host: Anser fabalis (Latham, 1787).

Ref.: Balát (1953, 1956, 1977).

Location: Čilistov, 5 Nov. 1949 (Balát Coll., MMBC slide number 1361).

\section{Anaticola beieri Eichler, 1954a}

Host: Branta ruficollis (Pallas, 1769).

Ref.: Balát (1956, 1977).

Location: Dunajská Streda, 10 Nov. 1953 (Balát 1956).

Anaticola crassicornis (Scopoli, 1763)

Host: Anas platyrhynchos Linnaeus, 1758. 
Ref.: Balát (1953) as Anaticola crassicorne; Balát (1956, 1977); Straka (1987).

Locations: Bratislava, 28 Dec. 1948 (Balát 1956); Martin, 20 Oct. 1977 and 20 Dec. 1982; Kl’ačany, 10 Dec. 1981; (Straka Coll., AKMM).

Note: Straka (1987) recorded data about collections of Anaticola crassicornis from Anas platyrhynchos and Spatula querquedula, but without mentioning which locality referred to each host species. Here we mention locations separately according to Straka's notes on relevant slides.

\section{Anaticola crassicornis (Scopoli, 1763)}

Host: Spatula clypeata (Linnaeus, 1758).

Ref.: Straka (1987) as Anaticola hopkinsi Eichler, 1954a.

Location: Michalovce, 30 Nov. 1977 (Straka Coll., AKMM)

Anaticola crassicornis (Scopoli, 1763)

Host: Spatula querquedula (Linnaeus, 1758).

Ref.: Balát (1953, 1956, 1977); Straka (1987).

Locations: Senné, 20 Apr. 1950 (Balát Coll., MMBC slide number 457); Dražkovce, 20 Apr. 1981 (Straka Coll., AKMM).

Note: Straka (1987) recorded data about collections of Anaticola crassicornis from Anas platyrhynchos and Spatula querquedula, but without mentioning which locality referred to each host species. Here we mention locations separately according to Straka's notes on relevant slides.

\section{Anaticola mergiserrati (De Geer, 1778)}

Host: Aythya fuligula (Linnaeus, 1758).

Ref.: this paper.

Location: Jakubov, 13 Jul. 1997 (Krištofík Coll., VETUNI).

Note: This is one of two first records of Anaticola mergiserrati from Slovakia (see below).

\section{Anaticola mergiserrati (De Geer, 1778)}

Host: Mergellus albellus (Linnaeus, 1758).

Ref.: this paper.

Location: Čilistov, 23 Mar. 1950 (Balát Coll., MMBC slide number 481).

Note: This is one of two first records of Anaticola mergiserrati from Slovakia (see above). This host-louse association was reported from the Danube Delta by Rékási et al. (2017), but it was not listed by Price et al. (2003).

\section{Anatoecus dentatus (Scopoli, 1763)}

Host: Anas platyrhynchos Linnaeus, 1758.

Ref.: Balát (1953, 1956, 1977).

Locations: Slovakia, 10 Nov. 1948 (Balát Coll., MMBC slide number 357); Bratislava, 10 Oct. 1948 and 28 Dec. 1948; Sládkovičovo, 16 Oct. 1951 (Balát 1956).

\section{Anatoecus dentatus (Scopoli, 1763)}

Host: Anser fabalis (Latham, 1787).

Ref.: Balát (1956) as Anatoecus sp.

Location: Čilistov, 5 Nov. 1949 (Balát Coll., MMBC slide number 1360).

\section{Anatoecus dentatus (Scopoli, 1763)}

Host: Aythya ferina (Linnaeus, 1758).

Ref.: this paper.

Location: Žitný ostrov, 7 May 1938 (Pfleger Coll., NMPC).

Note: This is a new host-louse association for Slovakia.

Anatoecus dentatus (Scopoli, 1763)

Host: Branta ruficollis (Pallas, 1769). 
Ref.: Balát $(1956,1977)$ as Anatoecus brantae Eichler, 1946; Hudec \& Černý (1972) as Anatoecus icterodes (Nitzsch. 1818).

Location: Dunajská Streda, 10 Nov. 1953 (Balát 1956).

Note: Although we could not find any specimens with the above data, we follow Price et al. (2003: 144) and Grossi et al. (2014: 606) regarding both Anatoecus brantae and Anatoecus icterodes as junior synonyms of Anatoecus dentatus.

\section{Anatoecus dentatus (Scopoli, 1763)}

Host: Cygnus olor (Gmelin, 1789).

Ref.: Straka (1987) as Anatoecus icterodes (Nitzsch. 1818).

Location: Ďanová, 1 Mar. 1977 (Straka Coll., AKMM).

Anatoecus dentatus (Scopoli, 1763)

Host: Mergellus albellus (Linnaeus, 1758).

Ref.: Balát $(1953,1956)$ as Anatoecus sp. ?icterodes.

Locations: Gabčíkovo, 24 Mar. 1954 (Balát Coll., MMBC slide number 481); Gabčíkovo, 24 Mar. 1954 (Balát 1956).

Note: We follow Grossi et al. (2014: 606) regarding Anatoecus icterodes as a junior synonym of Anatoecus dentatus.

\section{Anatoecus dentatus (Scopoli, 1763)}

Host: Mergus merganser Linnaeus, 1758.

Ref.: Balát (1977) as Anatoecus bipunctatus (Giebel, 1874).

Location: Slovakia (Balát 1977).

Notes: Balát (1977) recorded Anatoecus bipunctatus from Slovakia without a host association. Since Mergus merganser is the type host of $A$. bipunctatus (see Price et al. 2003: 144), we assume that Balát's record was from this host. Price et al. (2003: 144) synonymised this species with Anatoecus icterodes, which was subsequently regarded as a junior synonym of Anatoecus dentatus by Grossi et al. (2014: 606).

\section{Anatoecus dentatus (Scopoli, 1763)}

Host: Spatula querquedula (Linnaeus, 1758).

Ref.: Balát (1953, 1956, 1977).

Locations: Senné, 20 Apr. 1950 (Balát Coll., MMBC slide number 457).

\section{Anatoecus sp.}

Host: Anser brachyrhynchus Baillon, 1834.

Ref.: Balát (1956).

Location: Svätojurský Šúr, 24 Oct. 1953 (Balát 1956).

Notes: We could not find any specimens with the above data. Therefore, this record of Anatoecus from Anser brachyrhynchus needs confirmation with other material, to determine whether Anatoecus is a natural parasite of this host or if that record was due to accidental straggling.

\section{Aquanirmus bahli Tandan, 1951}

Host: Tachybaptus ruficollis (Pallas, 1764).

Ref.: Straka (1987) as A. [runcinatus] bahli Tandan, 1951.

Location: Turany, 11 Sep. 1983 (Straka Coll., AKMM).

Aquanirmus colymbinus (Scopoli, 1763)

Host: Podiceps auritus (Linnaeus, 1758).

Ref.: Balát (1953, 1956, 1977).

Location: Pezinok, Nov.-Dec. 1948 (Balát Coll., MMBC slide number 348).

Notes: There are only two nymphs on a slide in the Balát Collection, which are not suitable for a species identifica- 
tion. Therefore, considering that Podiceps auritus is the type host of Aquanirmus colymbinus, we accept Balát's identification as reported in his papers.

\section{Aquanirmus podicepis (Denny, 1842)}

Host: Podiceps cristatus (Linnaeus, 1758).

Ref.: Balát (1953) as A. colymbinus (Scopoli, 1763); Balát (1956, 1977).

Location: Gabčíkovo, 22 Jul. 1953 (Balát 1956).

Notes: We could not find any specimens with the above data but, considering that Podiceps cristatus is the type host of Aquanirmus podicepis, we accept Balát's identification as reported in his papers dated 1956 and 1977.

Ardeicola ardeae (Linnaeus, 1758)

Host: Ardea cinerea Linnaeus, 1758.

Ref.: Balát (1953, 1956, 1977).

Locations: Bratislava, 18 Mar. 1949 (Balát Coll., SNMB slide number 549); Slovakia, 19 Nov. 1950 (Balát 1956).

\section{Ardeicola ciconiae (Linnaeus, 1758)}

Host: Ciconia ciconia (Linnaeus, 1758).

Ref.: Balát (1977) as Ardeicola ardealis (Scopoli, 1763).

Location: Slovakia (Balát 1977).

Notes: Balát (1977) recorded Ardeicola ardealis from Slovakia without a host association. Considering that Ciconia ciconia is the type host of A. ardealis, we assume that this is the host of Balát's record. Although we could not find any specimens with the above data, we follow Price et al. (2003: 148) regarding Ardeicola ardealis as a junior synonym of Ardeicola ciconiae.

\section{Ardeicola expallidus Blagoveshtchensky, 1940}

Host: Ardea alba Linnaeus, 1758.

Ref.: Balát (1953) as Ardeicola sp.; Balát (1956, 1977) as Ardeicola albulus Eichler, 1948.

Location: Dunajská Streda, 19 Aug. 1946 (Balát Coll., MMBC slide number 522).

Notes: Balát (1956) recorded the date as "19 Aug. 1949" but, according to Balát's notes, the correct date is "19 Aug. 1946”. We follow Price et al. (2003: 148) regarding Ardeicola albulus as a junior synonym of A. expallidus.

Ardeicola rhaphidius (Nitzsch [in Giebel], 1866)

Host: Plegadis falcinellus (Linnaeus, 1758).

Ref.: Straka (1987).

Location: Vrútky, 2 Oct. 1977 (Straka Coll., AKMM).

\section{Ardeicola stellaris (Denny, 1842)}

Host: Botaurus stellaris (Linnaeus, 1758).

Ref.: Balát (1953, 1956, 1977); Straka (1987).

Locations: Podunajské Biskupice, 3 Dec. 1950; Slovakia, May 1948 (Balát Coll., MMBC slide numbers 87, 5562x); Ivánka pri Dunaji, 14 Oct. 1951 (Balát 1956); Lipovec, 10 Nov. 1980 (Straka Coll., AKMM).

Note: Balát (1956) recorded the date of "Podunajské Biskupice" as "3 Jul. 1950" but, according to Balát's notes, the correct date is "3 Dec. 1950".

\section{Brueelia antimarginalis Eichler, 1951b}

Host: Turdus pilaris Linnaeus, 1758.

Ref.: Balát (1956, 1977); Straka (1987).

Locations: Podhradie, 30 Oct. 1950 (Balát Coll., MMBC slide number 514); Sučianská dolina, 17 Jul. 1977 (Straka Coll., AKMM - not present in the collection).

Brueelia balati Krištofik, 1999

Host: Remiz pendulinus (Linnaeus, 1758). 
Ref.: Krištofík (1999).

Location: Jakubov, 13 Aug. 1989 (Krištofík 1999).

\section{Brueelia blagovescenskyi Balát, 1955b}

Host: Emberiza schoeniclus (Linnaeus, 1758).

Ref.: this paper.

Location: Gbelce, 13 Apr.-2 May 2008 (VETUNI).

Note: This is the first record of Brueelia blagovescenskyi from Slovakia.

\section{Brueelia breueri Balát, 1955b}

Host: Chloris chloris (Linnaeus, 1758).

Ref.: Balát (1955b, 1956, 1977); Gustafsson \& Bush (2017); Gustafsson et al. (2019).

Locations: Gabčíkovo, 25 Mar. 1954; Podunajské Biskupice, 20 Jul. 1953 (Balát Coll., MMBC slide numbers 676, 1118-3x, NHML slide number B.M: 1955-662-2x); Bzenica, 20 Jun. 1953 (Balát 1956).

\section{Brueelia chrysomytris (Blagoveshtchenky, 1940)}

Host: Spinus spinus (Linnaeus, 1758).

Ref.: Balát (1955b, 1956, 1977); Bush et al. (2018).

Locations: Sklené Teplice, 8 Oct. 1953 (Balát Coll., MMBC slide number 1035); Tatranská Javorina, Jun.-Jul. 2015 (Bush et al. 2018).

\section{Brueelia conocephala (Blagoveshtchensky, 1940)}

Host: Sitta europaea Linnaeus, 1758.

Ref.: Balát (1956, 1977); Gustafsson et al. (2019).

Location: Košice, 5 Nov. 1953 (Balát Coll., MMBC slide number 1080-17x).

\section{Brueelia cyclothorax (Burmeister, 1838)}

Host: Passer domesticus (Linnaeus, 1758).

Ref.: Balát (1956) as Brueelia sp.; Balát (1977).

Locations: Gabčíkovo, 22 Jul. 1953, 24 Mar. 1954; Palín, 5 Apr. 1956; Sklené Teplice, 20 Apr. 1953 (Balát Coll., MMBC slide numbers 1061, 1130, 1131, 1317, 1430-2x); Járok u Nitry, 17 Jun. 1953; Gabčíkovo, 17 Mar. 1954 (Balát 1956).

\section{Brueelia cyclothorax (Burmeister, 1838)}

Host: Passer montanus (Linnaeus, 1758).

Ref.: Balát (1953, 1956, 1977); Straka (1987); this paper.

Locations: Gabčíkovo, 22 Jul. 1953; Podunajské Biskupice, 21 Jul. 1953; Járok u Nitry, 17 Jun. 1953 (Balát Coll., MMBC slide numbers 1023, 1028, 1032, NHML slide number 321/53 Brit.Mus.1954-252); Sklené Teplice, 15 Apr. 1953; Járok u Nitry, 16-17 Jun. 1953; Gabčíkovo, 16-25 Mar. 1954; Hrhov, 31 Oct. 1953; Šaca, 1 Nov. 1953 (Balát 1956); Šulany, 24 Apr. 1997; Hronovce, 13 Feb. 2001; Kopčany, 5 Jul. 2004 (Krištofík Coll., VETUNI); Kláštor pod Znievom, 13 Mar. 1981 (Straka Coll., AKMM).

\section{Brueelia delicata (Nitzsch [in Giebel], 1866)}

Host: Emberiza citrinella Linnaeus, 1758.

Ref.: Balát (1955b, 1956, 1977); this paper.

Locations: Košice, 29 Oct. 1953 (Balát Coll., MMBC slide number 1100); Gabčíkovo, 6 May 1954 (Balát 1956); Čunovo, 26 Jun. 2001 (Krištofík Coll., VETUNI).

\section{Brueelia imponderabilica Eichler, 1954b}

Host: Lanius excubitor excubitor Linnaeus, 1758.

Ref.: Balát (1977); Szczykutowicz et al. (2006) as Brueelia cruciata (Burmeister, 1838); Gustafsson \& Bush (2017); this paper. 
Locations: Slovakia (Balát 1977); NE Slovakia (Szczykutowicz et al. 2006); Hniezdne, 25 Mar. 1964 (Weisz Coll., VETUNI).

Notes: Balát (1977) recorded this species from Slovakia without a host association. Since this species parasitises Lanius excubitor only (Price et al. 2003: 155), it is most likely that Balát's (1977) record refers to this host association.

\section{Brueelia intermedia (Nitzsch [in Giebel], 1866)}

Host: Turdus torquatus Linnaeus, 1758.

Ref.: Balát (1977); Hudec (1983); Straka (1987).

Locations: Slovakia (Balát 1977); Vrícko, 25 May 1978 (Straka Coll., AKMM).

Notes: Balát (1977) recorded this species from Slovakia without a host association. Hudec (1983) recorded this louse species from Turdus torquatus, but without giving a location. Gustafsson \& Bush (2017: 410) resurrected Brueelia intermedia from synonymy regarding it as a valid species and differing from $B$. marginata. Considering that B. intermedia is specific to T. torquatus only (Gustafsson \& Bush 2017: 348) and that Balát (1977) listed both B. intermedia and B. marginata (see below under Guimaraesiella marginata), we regard B. intermedia as recorded from Slovakia.

\section{Brueelia juno (Giebel, 1874)}

Host: Coccothraustes coccothraustes (Linnaeus, 1758).

Ref.: Balát (1956, 1977).

Locations: Banská Štiavnica-Počúvadla, 21 Jun. 1953; Járok u Nitry, 17 Jun. 1953 (Balát Coll., MMBC slide numbers 1115,1116$)$.

\section{Brueelia kluzi Balát, 1955b}

Host: Fringilla coelebs Linnaeus, 1758.

Ref.: Bush et al. (2018); this paper.

Locations: Tatranská Javorina, Jun.-Jul. 2015 (Bush et al. 2018); Vel'ké Blahovo, 15 May 2010 (Krištofík Coll., VETUNI).

\section{Brueelia kratochvili Balát, 1958}

Host: Motacilla alba Linnaeus, 1758.

Ref.: Gustafsson et al. (2019).

Location: Krišovská Liesková-Krížany, 14 Apr. 1959 (Balát Coll., MMBC slide numbers 1215, 1216, 1217).

\section{Brueelia kratochvili Balát, 1958}

Host: Motacilla flava Linnaeus, 1758.

Ref.: Balát (1977); Gustafsson et al. (2019).

Location: Vel'ké Kapušany, 18 Apr. 1959 (Balát Coll., MMBC slide numbers 1485, 1486, 1487-3x, 1488-3x).

\section{Brueelia locustellae Fedorenko, 1975}

Host: Locustella luscinioides (Savi, 1824).

Ref.: this paper.

Location: Gbelce, 21 Apr.-1 May 2008, 19 Apr. 2009, 17 Apr. 2016, 10 Jul. 2019 (VETUNI).

Note: This is the first record of Brueelia locustellae from Slovakia.

\section{Brueelia nebulosa (Burmeister, 1838)}

Host: Sturnus vulgaris Linnaeus, 1758.

Ref.: Balát (1956, 1977); this paper.

Locations: Banská Štiavnica—Počúvadla, 23 Apr. 1953; Kláštor pod Znievom, 10 May 1956 (Balát Coll., MMBC slide numbers 832-12x, 1126); Gbelce, 13 Apr.-1 May 2008, 17 Apr. 2016, 17 Apr. \& 2 Oct. 2019 (VETUNI). 
Brueelia parviguttata (Blagoveshtchensky, 1940)

Host: Alauda arvensis Linnaeus, 1758.

Ref.: Balát (1977); Straka (1987); this paper.

Locations: Gabčíkovo, 24 Mar. 1954, Slovakia (Balát 1977); Kláštor pod Znievom, 13 Mar. 1981 (Straka Coll., AKMM); Dunajská Lužná, 25 May 1997 (Krištofík Coll., VETUNI).

Note: Brueelia parviguttata parasitises two host species: Alauda arvensis and Galerida cristata (see Gustafsson \& Bush 2017: 351), but our recent material confirms A. arvensis as a host for this louse species in Slovakia.

\section{Brueelia pyrrhularum Eichler, 1954b}

Host: Pyrrhula pyrrhula pyrrhula (Linnaeus, 1758).

Ref.: Balát (1955b, 1956, 1977); Straka (1987); Gustafsson \& Bush (2017); this paper.

Locations: Gabčíkovo, 24 Mar. 1954 (Balát Coll., MMBC slide number 687); Vel'ká Ida, 28 Oct. 1953 (Balát 1956); Martin, 2 Feb. 1981; Vrícko, 20 Feb. 1983 (Straka Coll., AKMM); Bratislava-Mudroňova, 16 Jan. 2011 (Krištofík Coll., VETUNI).

Note: Straka (1987) recorded the date of collection as "Vrícko, 22 Feb. 1983" but, according to Straka's note on label of an available slide, the correct date is probably "20 Feb. 1983".

\section{Brueelia rosickyi Balát, 1955b}

Host: Sylvia nisoria (Bechstein, 1792).

Ref.: Balát (1955b, 1956, 1977); Gustafsson et al. (2019).

Location: Járok u Nitry, 17 Jun. 1953 (Balát Coll., MMBC slide number 1070).

\section{Brueelia stadleri Eichler, 1954b}

Host: Linaria cannabina (Linnaeus, 1758).

Ref.: Straka (1987) as "Brueelia (densilimba) stadleri".

Location: ŠPR Kláštorské lúky pri Kláštore pod Znievom, 18 Mar. 1981 (Straka Coll., AKMM).

\section{Brueelia straminea (Denny, 1842)}

Host: Dendrocopos leucotos (Bechstein, 1802).

Ref.: Balát (1953) as Picicola stramineus; Balát (1956) as Brueelia sp.; Straka (1987) as Picicola fixa Złotorzycka, 1964a.

Locations: Košice, 15 Jan. 1949 (Balát Coll., MMBC slide number 378); Martin, 27 Jan. 1977 (Straka Coll., AKMM-not present in the collection).

\section{Brueelia straminea (Denny, 1842)}

Host: Dendrocopos major (Linnaeus, 1758).

Ref.: Balát (1953) as Picicola stramineus; Balát (1956, 1977); Straka (1987); this paper.

Locations: Gabčíkovo, 16-20 Mar. \& 10 Oct. 1954 (Balát 1956); Martin, 2 Dec. 1981; Kláštor pod Znievom, 7 Mar. 1979, 25 Jan. 1983 (Straka Coll., AKMM); Bratislava, 28 Feb. 1997 (Krištofík Coll., VETUNI).

\section{Brueelia vaneki Balát, 1981 a}

Host: Acrocephalus schoenobaenus (Linnaeus, 1758).

Ref.: this paper.

Location: Gbelce, 16 Apr.-3 May 2008, 24 Apr.-1 May 2009 (VETUNI).

Note: This is the first record of Brueelia vaneki from Slovakia.

\section{Brueelia sp.}

Host: Acrocephalus scirpaceus (Hermann, 1804).

Ref.: this paper.

Location: Gbelce, 14-23 Apr. 2008, 20-23 Apr. 2009 (VETUNI).

Notes: This is the first record of Brueelia from Acrocephalus scirpaceus. As our specimens are not suitable for a species identification, more material is necessary to confirm if Brueelia is a natural parasite of this host or if this record is due to contamination or straggling. 


\section{Brueelia sp.}

Host: Lanius minor Gmelin, 1788.

Ref.: this paper.

Location: Zemplínska Široká—Rebrín, 4 Aug. 1959 (Balát Coll., MMBC slide numbers 1494—not present in the collection).

Notes: According to Gustafsson \& Bush (2017: 412) Lanius minor is host to Brueelia minor Lunkaschu, 1970. Since we have not found any specimens of Brueelia from L. minor in the Balát Collection, we cannot confirm this record to species level.

\section{Brueelia sp.}

Host: Saxicola rubetra (Linnaeus, 1758).

Ref.: this paper.

Location: Velké Leváre-NPR Abrod, 18 May 1974 (Balát Coll., MMBC slide number 1473).

Notes: This is the first record of Brueelia from Saxicola rubetra. As our single specimen is not suitable for a species identification, more material is necessary to confirm if Brueelia is a natural parasite of this host or if this record is due to contamination or straggling.

Campanulotes bidentatus (Scopoli, 1763)

Host: Columba palumbus Linnaeus, 1758.

Ref.: this paper.

Location: Sládkovičovo, 24 Jun. 1997 (Krištofík Coll., VETUNI).

Note: This is the first record of Campanulotes bidentatus from Slovakia.

\section{Campanulotes compar (Burmeister, 1838)}

Host: Columba livia Gmelin (1789).

Ref.: Straka (1987).

Location: Martin, 24 Jan. 1977 and 15 Dec. 1976 (Straka Coll., AKMM).

Note: Straka (1987) recorded the date of collection as "15 Dec. 1977" but, according to Straka's notes on labels of both available slides, the correct date is probably "15 Dec. 1976".

\section{Capraiella subcuspidata (Burmeister, 1838)}

Host: Coracias garrulus Linnaeus, 1758.

Ref.: Balát (1956, 1977).

Locations: Bratislava, Jul. 1951; Slovakia, Summer of 1952; Šamorín, 14 Sep. 1951 (Balát Coll., MMBC slide numbers 622, 708, SNMB slide number 663).

\section{Carduiceps scalaris (Piaget, 1880)}

Host: Calidris pugnax (Linnaeus, 1758).

Ref.: this paper.

Location: Štrba, 21 Mar. 1936 (Pfleger Coll., SNMB).

Note: This is the first record of Carduiceps scalaris from Slovakia.

\section{Cincloecus cincli (Denny, 1842)}

Host: Cinclus cinclus (Linnaeus, 1758).

Ref.: Balát (1953, 1955a, 1956, 1977) as Philopterus cincli; Straka (1987) as Philopterus cincli.

Locations: Trenčianské Teplice, 11 Jun., 13-18 Jun. 1950 (Balát Coll., MMBC slide numbers 529, 532a, 536, SNMB slide number 532b); Javorová dolina, 12 May 1952; Žarnovica, 7 Jan. 1952; Sklené Teplice, 14 Apr., 6-11 Oct. 1953; Žiar nad Hronom, formerly Svätý Kríž nad Hronom—dolina Kl’ak, 16 Apr. 1953 (Balát 1955a, 1956); Kláštorské lúky pri Kláštore pod Znievom, 16 Jan. 1981 (Straka Coll., AKMM).

Coloceras damicorne (Nitzsch, 1866)

Host: Columba palumbus Linnaeus, 1758. 
Ref.: Balát (1953, 1956, 1977); Straka (1987).

Locations: Gabčíkovo, 13 Mar. 1954 (Balát 1956); Martin, 5 Jul. 1979) (Straka Coll., AKMM).

Coloceras piageti (Johnston \& Harrison, 1912)

Host: Streptopelia decaocto (Fryvaldszky, 1838).

Ref.: Straka (1987) as Coloceras sofioticus Eichler, 1950.

Locations: Slovany, 15 Aug. 1978 (Straka Coll., AKMM).

\section{Columbicola bacillus (Giebel, 1866)}

Host: Streptopelia decaocto (Frivaldszky, 1838).

Ref.: Balát (1977) as Columbicola confusissimus Eichler, 1947; Straka (1987) as C. confucissimus.

Locations: Vojany, 17 Apr. 1959 (Balát Coll., MMBC slide number 1178); Slovany, 15 Aug. 1978 (Straka Coll., AKMM).

\section{Columbicola bacillus (Giebel, 1866)}

Host: Streptopelia turtur (Linnaeus, 1758).

Ref.: Balát (1953, 1956) as Columbicola baculus bacillus; Balát (1977).

Location: Járok u Nitry, 16 Jun. 1953 (Balát 1956).

\section{Columbicola claviformis (Denny, 1842)}

Host: Columba palumbus Linnaeus, 1758.

Ref.: this paper.

Locations: Sládkovičovo, 24 Jun. 1997; Štvrtok na Ostrove, 17 Jul. 2005 (Krištofík Coll., VETUNI).

Note: This is the first record of Columbicola claviformis from Slovakia.

Columbicola columbae (Linnaeus, 1758)

Host: Columba livia Gmelin, 1789.

Ref.: Straka (1987).

Locations: Martin, 24 Jan. 1977, 15 Dec. 1976, 12 Jul 1977 and 5 Jul. 1979 (Straka Coll., AKMM).

Note: Straka (1987) recorded the date of collection as "15 Dec. 1977" but, according to Straka's notes on labels of both available slides, the correct date is probably "15 Dec. 1976". Columba palumbus is noted as host of Columbicola on the label of single slide from 5 Jul. 1979. It was determined by Straka as C. columbae. However, C. palumbus is host of Columbicola claviformis (Price et al. 2003). Unfortunately, we can not confirm if this record represents another natural occurrence of $C$. claviformis or accidental straggler of $C$. columbae on atypical host, because this specimen is in poor condition. Therefore we accept Straka's conclusion to not mention $C$. palumbus neither as host of C. claviformis nor C. columbae.

\section{Corvonirmus tasniemae (Ansari, 1957)}

Host: Corvus frugilegus Linnaeus, 1758.

Ref.: Balát (1977) as Brueelia tasniemae; Straka (1987).

Locations: Slovakia (Balát 1977); Stará Bystrica, 5 Jan. 1982; Slovany, 20 Dec. 1982 (Straka Coll., AKMM).

Notes: Balát (1977) recorded this species from Slovakia without a host association. Considering that Corvus frugilegus is the type host of Corvonirmus tasniemae (see Price et al. 2003: 159), we assume that $C$. frugilegus is the host of Balát's record. Gustafsson \& Bush (2017: 355) transferred Brueelia tasniemae to the genus Corvonirmus.

\section{Corvonirmus uncinosus (Burmeister, 1838)}

Host: Corvus cornix Linnaeus, 1758.

Ref.: Balát $(1956,1977)$ as Brueelia (Corvonirmus) uncinosa), this paper.

Locations: Bojnice, 4 May 1953 (Balát Coll., MMBC slide number 778); Kláštor pod Znievom, 13 Mar. 1981 (Straka Coll., AKMM) 
Craspedonirmus colymbinus (Denny, 1842)

Host: Gavia arctica (Linnaeus, 1758).

Ref.: Balát (1953, 1956, 1977) as Craspedonirmus frontatus (Nitzsch [in Giebel], 1866); Straka (1987) as C. frontatus.

Locations: Š́rovce, Nov.-Dec. 1948 (Balát Coll., MMBC slide number 349—not present in the collection); Martin, 23 Mar. 1977; Krpel'any, 15 Nov. 1980 (Straka Coll., AKMM-any slide from Martin, 23 Mar. 1977 is not present in the collection).

\section{Craspedorrhynchus aquilinus (Denny, 1842)}

Host: Aquila chrysaetos (Linnaeus, 1758).

Ref.: Balát (1953, 1956, 1977); Straka (1987); this paper.

Locations: Bošany, 4 Jan. 1949 (Balát Coll., MMBC slide number 415); Slovakia, May 1950 (Balát 1956); Folkušová, 17 Feb. 1977, Martin, 30 May 1977 (Straka Coll., AKMM); Jovsa, 14 Sep. 1930; Tatry, 21 Oct. 1927 (Pfleger Coll., SNMB, NMPC, MMBC).

\section{Craspedorrhynchus dilatatus (Rudow, 1869b)}

Host: Buteo lagopus (Pontoppidan, 1763).

Ref.: Balát (1953) as Craspedorrhynchus buteo-lagopi Merisuo, 1945; Balát (1956, 1977); Straka (1987); this paper.

Locations: Slovakia, 18 Dec. 1946 (Balát Coll., MMBC slide number 717); Slovakia, Mar. 1947; Dunajská Streda, 3 Feb. 1949; Myslenice, 4 Feb. 1951; Bratislava, 16 Nov. \& 12 Dec. 1952 (Balát 1956); Martin, 24 Jan. 1977 ; Ležiachov, 13 Jan. 1979 (Straka Coll., AKMM); Trnava, 15 Feb. 1939 (Pfleger Coll., SNMB).

Note: Straka (1987) recorded the location and date as "Vrícko, 17 Jan. 1979", but it is most likely an error, because in Straka's note on label of an available slide the location and date are given as "Ležiachov, 13 Jan. 1979".

\section{Craspedorrhynchus haematopus (Scopoli, 1763)}

Host: Accipiter gentilis (Linnaeus, 1758).

Ref.: this paper.

Locations: Čabrad u Krupiny, 25 Jan. 1938 (Pfleger Coll., SNMB, NMPC).

Note: This is the first record of Craspedorrhynchus haematopus from Slovakia.

Craspedorrhynchus macrocephalus (Nitzsch [in Giebel], 1874)

Host: Haliaeetus albicilla (Linnaeus, 1758).

Ref.: Balát (1953, 1956, 1977).

Location: Šal'a, 19 Mar. 1951 (Balát 1956).

Craspedorrhynchus melittoscopus (Nitzsch [in Giebel], 1874)

Host: Pernis apivorus (Linnaeus, 1758).

Ref.: Balát (1953, 1956, 1977).

Location: Pezinok, 5 May 1949) (Balát 1956).

\section{Craspedorrhynchus naevius (Giebel, 1861)}

Host: Clanga pomarina (Brehm, 1831).

Ref.: Balát (1956, 1977); this paper.

Locations: Vráble, 24 Aug. 1952 (Balát 1956); Slovakia (Pfleger Coll., SNMB).

\section{Craspedorrhynchus platystomus (Burmeister, 1838)}

Host: Buteo buteo (Linnaeus, 1758).

Ref.: Balát (1953, 1956, 1977); Straka (1987); this paper.

Locations: Bratislava_Petržalka, 1 Sep. 1949; Košice, 16 Nov. 1948 (Balát Coll., MMBC slide numbers 339, 359, SNMB slide number 530); Budmerice, 20 Mar. 1949; Plavecké Podhradie, 5 Nov. 1950 (Balát 1956); Martin, 18 Jan. 1977; Lipovec, 6 and28 Mar. 1977; Kláštor pod Znievom, 18 May 1978 (Straka Coll., AKMM); Váhovce, 
20 Mar. 2001; Moravský Svätý Ján, 17 Dec. 2009; Závod, 11 Mar. 2010; Malé Leváre, 16 Mar. 2010 (Krištofík Coll., VETUNI).

Note: Straka (1987) recorded data on collections of Craspedorrhynchus platystomus from Buteo buteo and Buteo rufinus, but without mentioning which locality referred to each host species. Here we mention locations separately according to Straka's notes on relevant slides. Straka (1987) recorded one of the location and dates for this host as "Lipovec, 6 and 28 Mar. 1977", but it is most likely an error, because in Straka's notes on labels of four available slides with aforementioned dates the location is given as "Česká Lípa, Czech Republic". Despite it we also list this location, because we cannot completely exclude the possibility that material from this location is only not present in the collection.

\section{Craspedorrhynchus platystomus (Burmeister, 1838)}

Host: Buteo rufinus (Cretzschmar, 1829).

Ref.: Straka (1987).

Location: Martin, 17 Feb. 1977 (Straka Coll., AKMM — not present in the collection).

Note: Straka (1987) recorded data on collections of Craspedorrhynchus platystomus from Buteo buteo and Buteo rufinus, but without mentioning which locality referred to each host species. According to Straka's notes on relevant slides lice form Buteo rufinus were collected on 17 Feb. 1977 in Martin. No louse with the same location and date from Buteo buteo is in the collection. On the other hand, all six available slides with lice from $B$. rufinus contain Degeeriela fulva (see below). According to Straka's notes on these slides, all these lice were identified as "Picicola sp.". Despite it we decide to accept Straka's record and list C. platystomus from B. rufinus, because lice of these two genera (Craspedorrhynchus vs. Degeeriela) are too different to be misidentify, and because we cannot completely exclude the possibility that material of this species is only not present in the collection. Although Price et al. (2003) did not list this host-louse association, Straka (1987) reported it from Slovakia, and Dik \& Aydenizöz-Ozkayhan (2007) confirmed it in Turkey.

\section{Craspedorrhynchus sp.}

Host: Aquila heliaca Savigny, 1809.

Ref.: Balát (1956) as Craspedorrhynchus sp.

Location: Štúrovo, 3 Feb. 1950 (Balát 1956).

Notes: According to Price et al. (2003: 169) Aquila heliaca is parasitised by Craspedorrhynchus fraterculus Eichler \& Złotorzycka, 1975. However, as there are no specimens of Craspedorrhynchus from this host in the Balát Collection, we cannot confirm the identity of this species.

\section{Cuclotogaster heterogrammicus (Nitzsch [in Giebel], 1866)}

Host: Perdix perdix (Linnaeus, 1758).

Ref.: Balát (1956, 1977).

Location: Trnava, 7 Oct. 1951 (Balát Coll., MMBC slide number 618).

\section{Cuclotogaster heterographus (Nitzsch [in Giebel], 1866)}

Host: Gallus gallus (Linnaeus, 1758) — captive bird.

Ref.: this paper.

Location: Martin, 19 Apr. 1977 (Straka Coll., AKMM).

Note: This is the first record of Cuclotogaster heterographus from Slovakia.

\section{Cuclotogaster heterographus (Nitzsch [in Giebel], 1866)}

Host: Phasianus colchicus Linnaeus, 1758.

Ref.: this paper.

Location: Slovakia, 10 Nov. 1948 (Balát Coll., MMBC slide number 355).

Note: Together with the above, this is the first record of Cuclotogaster heterographus from Slovakia.

Cuculicola latirostris (Burmeister, 1838)

Host: Cuculus canorus Linnaeus, 1758. 
Ref.: Straka (1987); this paper.

Locations: Kevice, 4 Aug. 1982 (Straka Coll., AKMM); Žitný ostrov, 29 Apr. 1938 (Pfleger Coll., NMPC).

\section{Cuculoecus latifrons (Denny, 1842)}

Host: Cuculus canorus Linnaeus, 1758.

Ref.: Balát (1953, 1956, 1977); this paper.

Locations: Plavecké Podhradie, 29 Apr. \& 6 May 1951 (Balát 1956); Žitný ostrov, 29 Apr. 1938 (Pfleger Coll., SNMB).

\section{Cummingsiella aurea Hopkins, 1949}

Host: Scolopax rusticola Linnaeus, 1758.

Ref.: Straka (1987); this paper.

Locations: Bratislava, 19 Mar. 1950 (Balát Coll., MMBC slide number 533); Vrícko, 1 May 1977; Valča, 1 May 1977; Kláštor pod Znievom, 22 Mar. 1977; Belá nad Cirochou, 4 May 1977; Bystrička, 3 Apr. 1978; Martin, 6 Apr. 1982 (Straka Coll., AKMM — any slide from Belá nad Cirochou, 4 May 1977 is not present in the collection).

\section{Cummingsiella ovalis (Scopoli, 1763)}

Host: Numenius arquata (Linnaeus, 1758).

Ref.: Balát (1977); this paper.

Locations: Malacky, 5 Jun. 1950; Senné, 16 Apr. 1950; Zohor, 16 May 1948 (Balát Coll., MMBC slide numbers 93, 458, 501, SNMB slide number 93-2x).

\section{Degeeriella aquilarum Eichler, 1943c}

Host: Clanga pomarina (Brehm, 1831).

Ref.: Balát (1977) as Degeeriella discocephalus aquilarum; this paper.

Location: Štrba, 2 Sep. 1938 (Pfleger Coll., SNMB).

Notes: Balát (1977) recorded Degeeriella discocephalus aquilarum from Slovakia without a host association. This louse species parasitises six species of eagles (Price et al. 2003: 173) of which Aquila chrysaetos, A. heliaca, Clanga clanga and C. pomarina may occur in Slovakia (Hudec \& Černý 1977). No specimen of Degeeriella from these hosts can be found in the Balát Collection but, as Balát was in contact with Pfleger, he may have examined lice from the Pfleger Collection. Therefore, we assume that Balát' (1977) record was based on material from the Pfleger Collection.

\section{Degeeriella discocephalus (Burmeister, 1838)}

Host: Haliaeetus albicilla (Linnaeus, 1758).

Ref.: Balát (1953, 1956, 1977).

Locations: Košice, Jan. 1949 (Balát Coll., MMBC slide number 310); Šal’a, 19 Mar. 1951; Šamorín, 19 Dec. 1952 (Balát 1956).

\section{Degeeriella fulva (Giebel, 1874)}

Host: Aquila chrysaetos (Linnaeus, 1758).

Ref.: Straka (1987).

Location: Martin, 17 Feb. 1977 (Straka Coll., AKMM).

\section{Degeeriella fulva (Giebel, 1874)}

Host: Buteo buteo (Linnaeus, 1758).

Ref.: Balát (1956) as Degeeriella giebeli, Hopkins, 1947; Balát (1977); Straka (1987) as D. giebeli; this paper.

Locations: Bratislava_Petržalka, 1 Sep. 1949 (Balát Coll., SNMB slide number 530); Budmerice, 20 Mar. 1949; Bratislava, 11 Nov. 1951; Bojnice, 28 Apr. 1953; Košice, 28 Oct. 1953 (Balát 1956); Martin, 18 Jan. 1977; Kláštor pod Znievom, 18 May 1978; Slovany, 15 Aug. 1978 (Straka Coll., AKMM); Sap, 20 Jan. 1998; Farná, 13 Feb. 2001; Váhovce, 20 Mar. 2001; Závod, 11 Mar. 2010; Malé Leváre, 16 Mar. 2010 (Krištofík Coll., VETUNI). 


\section{Degeeriella fulva (Giebel, 1874)}

Host: Buteo lagopus (Pontoppidan, 1763).

Ref.: Balát (1956) as Degeeriella angusta (Giebel, 1874); Balát (1977); this paper.

Locations: Slovakia, 18 Dec. 1946 (Balát Coll., MMBC slide number 718); Myslenice, 4 Feb. 1951 (Balát 1956); Trnava, 15 Feb. 1939 (Pfleger Coll., SNMB).

\section{Degeeriella fulva (Giebel, 1874)}

Host: Buteo rufinus (Cretzschmar, 1829).

Ref.: Balát (1956) as Degeeriella sp., this paper.

Locations: Slovakia, May 1948 (Balát Coll., MMBC slide number 22); Martin, 17 Feb. 1977 (Straka Coll., AKMM).

\section{Degeeriella fusca (Denny, 1842)}

Host: Circus aeruginosus (Linnaeus, 1758).

Ref.: Balát (1953) as Kélerinirmus fuscus (Denny, 1842); Balát (1956, 1977).

Locations: Bratislava-Rača, 4 Sep. 1948 (Balát Coll., MMBC slide number 303); Modra, 2 Sep. 1951; Vrakuň, 31 Aug. 1952 (Balát 1956).

\section{Degeeriella fusca (Denny, 1842)}

Host: Circus cyaneus (Linnaeus, 1766).

Ref.: Balát (1953) as Kelerinirmus fuscus (Denny, 1842); Balát (1956, 1977).

Locations: Bratislava, 13 Dec. 1948; Bratislava-Rača, 26 Jan. 1951; Gabčíkovo, 15 Oct. 1951; Lehnice, 2 Mar. 1952; Šal’a, 28 Jan. 1951; Velký Žitný Ostrov, 4 Jan. 1951 (Balát Coll., MMBC slide numbers 570, 585; 350, 588_-not present in the collection, SNMB slide numbers 606, 631); Č́ćov, 7 Oct. 1951 (Balát 1956).

\section{Degeeriella fusca (Denny, 1842)}

Host: Circus macrourus (Gmelin, 1770).

Ref.: Balát (1956, 1977).

Location: Slovakia, Oct. 1951 (Balát Coll., MMBC slide number 632).

Degeeriella leucopleura (Nitzsch [in Giebel], 1874)

Host: Circaetus gallicus (Gmelin, 1788).

Ref.: Balát (1953) as Kelerinirmus leucopleurus (Nitzsch [in Giebel], 1874); Balát (1956, 1977).

Location: Eastern Slovakia, Sep. 1949 (Balát 1956).

\section{Degeeriella nisus (Giebel, 1866)}

Host: Accipiter nisus (Linnaeus, 1758).

Ref.: Balát (1956, 1977); this paper.

Locations: Trnava, 28 Dec. 1950; Plavecký Mikuláš, 12 Aug. 1951 (Balát 1956); Bratislava, 2 Mar. 1997 (Krištofík Coll., VETUNI).

\section{Degeeriella regalis (Giebel, 1866)}

Host: Milvus migrans (Boddaert, 1783).

Ref.: Balát (1977); Hudec \& Černý (1977).

Location: Slovakia (Balát 1977).

Notes: Balát (1977) recorded Degeeriella regalis from Slovakia without a host association. This species parasitises ten species of raptors (Price et al. 2003: 175) of which Milvus migrans and Milvus milvus occur in Slovakia (Hudec \& Černý, 1977). Although, Hudec \& Černý (1977) recorded D. regalis from M. migrans, they gave no location. 


\section{Degeeriella rufa (Burmeister, 1838)}

Host: Falco cherrug Gray, 1834.

Ref.: Balát (1953) as Kélerinirmus rufus (Burmeister, 1838); Balát (1956, 1977); this paper.

Locations: Plavecké Podhradie, 17 Jun. 1950 (Balát 1956); Devínske jazero, 3 Jul. 2000 (Krištofík Coll., VETUNI).

\section{Degeeriella rufa (Burmeister, 1838)}

Host: Falco tinnunculus Linnaeus, 1758.

Ref.: Balát (1953) as Kélerinirmus rufus (Burmeister, 1838); Balát (1956, 1977); Straka (1987); this paper.

Locations: Čilistov, 19 Mar. 1950; Klenovec, 4 Feb. 1951; Trávnica, formerly Fíš, 2 May 1948 (Balát Coll., MMBC slide numbers 84-not present in the collection, 430, SNMB slide number 621); Trávnica, formerly Fíš, May 1948; Bratislava, 3 Apr. 1951 and Sep. 1952 (Balát 1956); Martin, 14 Jun. 1978 (Straka Coll., AKMM); Malacky, 13 May 2001; Král'ovičove Kračany, 26 May 2003 (Krištofík Coll.,VETUNI).

\section{Degeeriella rufa (Burmeister, 1838)}

Host: Falco vespertinus Linnaeus, 1766.

Ref.: Balát (1953) as Kelerinirmus quadraticollis (Rudow, 1870); Balát (1956) as Degeeriella quadraticollis; this paper.

Locations: Zlatná na Ostrove, 24 Aug. 1950 (Balát Coll., MMBC slide number 547); Senné, Jun. 1955 (Balát 1956); Šamorín, 10 Apr. 1925 (Pfleger Coll., SNMB, NMPC).

Degeeriella vagans (Giebel, 1874)

Host: Accipiter gentilis (Linnaeus, 1758).

Ref.: this paper.

Locations: Čabrad u Krupiny, 25 Jan. 1938 (Pfleger Coll., NMPC); Klačno, 10 Sep. 1985 (Straka Coll., AKMM).

Note: This is the first record of Degeeriella vagans from Slovakia.

Esthiopterum gruis (Linnaeus, 1758)

Host: Grus grus (Linnaeus, 1758).

Ref.: Balát (1956, 1977).

Locations: Senné, Spring of 1955 (Balát Coll., MMBC slide number 1003); Čilistov, 23 Mar. 1950 (Balát 1956).

Falcolipeurus sulcifrons (Denny, 1842)

Host: Haliaeetus albicilla (Linnaeus, 1758).

Ref.: Balát (1953, 1956, 1977); this paper.

Locations: Senné, Spring of 1955 (Balát 1956); Žitný ostrov, no date (Pfleger Coll., SNMB, NMPC, MMBC).

\section{Falcolipeurus suturalis (Rudow, 1869b)}

Host: Aquila chrysaetos (Linnaeus, 1758).

Ref.: Balát (1953) as Falcolipeurus sp.; Balát (1956, 1977).

Location: Čachtice, 1 Feb. 1949 (Balát Coll., MMBC slide numbers 416, 417).

\section{Fulicoffula gallinula Carriker, 1953}

Host: Gallinula chloropus (Linnaeus, 1758).

Ref.: this paper.

Location: Cífer, 2 May 1951 (Balát Coll., MMBC slide number 795).

Note: This is the first record of Fulicoffula gallinula from Slovakia.

\section{Fulicoffula lurida (Nitzsch, 1818)}

Host: Fulica atra Linnaeus, 1758.

Ref.: Straka (1987); this paper.

Locations: Krpel’any, 5 Jan. 1982 (Straka Coll., AKMM); Bratislava-Kopáč, 19 Feb. 1997 (Krištofík Coll., VETUNI). 


\section{Fulicoffula sp.}

Host: Rallus aquaticus Linnaeus, 1758.

Ref.: this paper.

Location: Gbelce, 27 Apr. 2009 (VETUNI).

Notes: Although we only examined nymphs collected from one ralliform bird, we are confident that they are not contaminants from another bird species. Therefore, we believe they are likely Fulicoffula rallina (Denny, 1842), with the type and only host, Rallus aquaticus (see Price et al. 2003: 181). This is a new host-louse association for Slovakia.

\section{Goniocotes chrysocephalus Giebel, 1874}

Host: Phasianus colchicus Linnaeus, 1758.

Ref.: Balát (1956, 1977); Goldová et al. (2006); this paper.

Locations: Bratislava-Rača, 31 Dec. 1950 (Balát Coll., MMBC slide number 568); Gabčíkovo, 21 Oct. 1954 (Balát 1956); Game Management Centre, Rozhanovce, 2000-2004 (Goldová et al. 2006); Studené, 27 Jul. 1998 (Krištofík Coll., VETUNI).

\section{Goniocotes gallinae (De Geer, 1778)}

Host: Gallus gallus (Linnaeus, 1758)—captive bird.

Ref.: this paper.

Location: Kláštor pod Znievom, 25 Apr. 1977 (Straka Coll., AKMM).

Note: Although the occurrence of this louse species if probably well known among veterinarians and hen breeders, this is the first record of Goniocotes gallinae from Slovakia. Specimens in the Straka's collection are mounted partially together with Menopon gallinae and Menacanthus stramineus on the same slides reported by Straka (1987) as Menacanthus sp.

\section{Goniocotes megalocephalus Uchida, 1916}

Host: Tetrastes bonasia (Linnaeus, 1758).

Ref.: this paper.

Location: Blatnica, 4 May 1979 (Straka Coll., AKMM).

Note: This is the first record of Goniocotes megalocephalus from Slovakia.

\section{Goniodes bituberculatus Rudow, 1869b}

Host: Tetrao urogallus Linnaeus, 1758.

Ref.: Balát (1953, 1956, 1977); Straka (1987).

Locations: Brezno, 20 Apr. 1949; Čierny Balog, 9 May 1956 (Balát Coll., MMBC slide numbers 1324, 1325); Brezno, 17 Apr. 1949 (Balát 1956); Martin, 29 Oct. 1981, Martinské hole, 12 May 1987 (Straka Coll., AKMM).

\section{Goniodes colchici Denny, 1842}

Host: Phasianus colchicus Linnaeus, 1758.

Ref.: Straka (1987); Goldová et al. (2006); this paper.

Locations: Martin, 7 Dec. 1977 (Straka Coll., AKMM); Game Management Centre, Rozhanovce, 2000-2004 (Goldová et al. 2006); Studené, 27 Jul. 1998 (Krištofík Coll., VETUNI).

Note: Straka (1987) reported Goniodes colchici from Phasianus colchicus from "Martin, 20 Jan. 1977", but it is most likely an error, because in Straka's notes on labels of two available slides with aforementioned date and location host is given as "jarabica pol'ná = Perdix perdix" (see below). Although we only examined one nymph from the Krištofík Collection, it is most likely Goniodes colchici, with the type and only host Phasianus colchicus (see Price et al. 2003: 183).

\section{Goniodes dispar Burmeister, 1838}

Host: Perdix perdix Linnaeus, 1758

Ref.: this paper.

Location: Martin, 20 Jan. 1977 (Straka Coll., AKMM). 
Notes: Straka (1987) reported Goniodes colchici from Phasianus colchicus from "Martin, 20 Jan. 1977", but it is most likely an error, because in Straka's notes on labels of two available slides with aforementioned date and location host is given as "jarabica pol'ná = Perdix perdix". According to Straka's notes on labels lice on these two slides were misidentified as "Goniodes colchici". This is the first record of Goniodes dispar from Slovakia.

\section{Goniodes dissimilis Denny, 1842}

Host: Gallus gallus (Linnaeus, 1758)—captive bird.

Ref.: Straka (1987).

Location: Martin, 19 Apr. 1979 (Straka Coll., AKMM).

Goniodes pavonis (Linnaeus, 1758)

Host: Pavo cristatus Linnaeus, 1758 - captive bird.

Ref.: Straka (1987).

Locations: Drážkovce, 14 Jan. 1985, Turčianská Štiavnička, 26 May 1979 (Straka Coll., AKMM).

\section{Goniodes tetraonis (Linnaeus, 1761)}

Host: Lyrurus tetrix (Linnaeus, 1758).

Ref.: Straka (1987).

Locations: Oravská priehrada pri Námestove, 1 May 1977; Oravský Podzámok, 1 May 1977; Sučany, 2 May 1977; Ratkovo, 6 May 1977 (Straka Coll., AKMM).

Notes: Straka (1987) recorded Tetrao urogallus and a hybrid between T. urogallus and Lyrurus tetrix as hosts of Goniodes tetraonis. It is known that T. urogallus and L. tetrix can produce hybrids in wild populations (Kleven et al. 2020), so finding lice on such a hybrid is both unusual and interesting. However, considering that T. urogallus is a natural host of Goniodes bituberculatus Rudow, 1869 (Price et al. 2003: 331; see above), the association between Goniodes tetraonis and T. urogallus is, in our opinion, questionable and most likely the result of contamination or straggling.

\section{Guimaraesiella amsel (Eichler, 1951b)}

Host: Turdus merula Linnaeus, 1758.

Ref.: Balát (1953) as Brueelia marginata; (Burmeister, 1838); Balát (1956, 1977) as Brueelia amsel; Bush et al. (2018).

Locations: Bratislava, 10 Feb. 1952 (Balát Coll., MMBC slide number 609); Tatranská Javorina, Jun.-Jul. 2015 (Bush et al. 2018).

\section{Guimaraesiella marginata (Burmeister, 1838)}

Host: Turdus pilaris Linnaeus, 1758.

Ref.: Balát (1953, 1956, 1977) as Brueelia marginata; Bush et al. (2018); Straka (1987) as Brueelia (Allobrueelia) marginata); this paper.

Locations: Bratislava_Petržalka, 9 Mar. 1952; Plavecké Podhradie, Jan. 1951; Plavecký Mikuláš, 31 Dec. 1950 (Balát Coll., MMBC slide numbers 566, 620; 591—not present in the collection); Necpaly, 25 Feb. 1967; Martin, 19 Apr. and 28 May 1979 (Straka Coll., AKMM); Tatranská Javorina, Jun.-Jul. 2015 (Bush et al. 2018); Štrbské pleso, 25 Jan. 1938 (Pfleger Coll., SNMB, NMPC).

\section{Hecatrishula varia (Burmeister, 1838)}

Host: Coloeus monedula (Linnaeus, 1758).

Ref.: this paper.

Location: Pavlovce nad Uhom, 9 Apr. 1956 (Balát Coll. Balát Coll., MMBC slide number 1186).

Notes: This is one of two first records of Hecatrishula varia from Slovakia (see below). This species was placed in the genus Brueelia Kéler, 1936, but Gustafsson \& Bush (2017: 88) transferred it to their new genus Hecatrishula. 


\section{Hecatrishula varia (Burmeister, 1838)}

Host: Corvus frugilegus Linnaeus, 1758.

Ref.: this paper.

Locations: Rohovce, 16 Mar. 1955 (Balát Coll. Balát Coll., MMBC slide number 780); Kravany, 9 Jun. 1998; Zlatná na Ostrove, 20 Jan. 2001 (Krištofík Coll., VETUNI).

Notes: This is one of two first records of Hecatrishula varia from Slovakia (see above). This species was placed in the genus Brueelia Kéler, 1936, but Gustafsson \& Bush (2017: 88) transferred it to their new genus Hecatrishula.

\section{Ibidoecus bisignatus (Nitzsch [in Giebel], 1866)}

Host: Plegadis falcinellus (Linnaeus, 1758).

Ref.: Straka (1987).

Location: Vrútky, 2 Oct. 1977 (Straka Coll., AKMM).

Note: Straka (1987) recorded the date of collection as "5 Oct. 1977" but, according to Straka's note on label of an available slide, the correct date is probably " 2 Oct. 1977 ".

\section{Ibidoecus plataleae (Denny, 1842)}

Host: Platalea leucorodia Linnaeus, 1758.

Ref.: Balát (1953, 1956, 1977); this paper.

Locations: Zlatná na Ostrove, 24 Aug. 1950 (Balát 1956); Šarluky, 18 Aug. 1929 (Pfleger Coll., SNMB, NMPC).

\section{Lagopoecus colchicus Emerson, 1949}

Host: Phasianus colchicus (Linnaeus, 1758).

Ref.: Straka (1987).

Locations: Martin, 6 Jan. 1977; Kremnica, 17 May 1978 (Straka Coll., AKMM).

\section{Lagopoecus lyrurus Clay, 1938}

Host: Lyrurus tetrix (Linnaeus, 1758).

Ref.: Balát (1953, 1956, 1977).

Location: Slovakia, May 1948 (Balát Coll., MMBC slide number 105).

Lagopoecus pallidovittatus (Grube, 1851)

Host: Tetrao urogallus Linnaeus, 1758.

Ref.: Balát (1953, 1956, 1977).

Locations: Brezno, 20 Apr. 1949; Čierny Balog, 9 May 1956 (Balát Coll., MMBC slide numbers 1324, 1325); Brezno, 17 Apr. 1949 (Balát 1956).

\section{Lagopoecus tetrastei Bechet, 1963}

Host: Tetrastes bonasia (Linnaeus, 1758).

Ref.: Balát (1977); this paper.

Location: Slovakia (Balát 1977); Blatnica, 4 May 1979 (Straka Coll., AKMM).

Notes: Balát (1977) recorded this species from Slovakia without a host association. Considering that Tetrastes bonasia is the type and only host of Lagopoecus tetrastei (see Price et al. 2003: 194), we assume that this is the host of Balát's record. Furthermore, the Straka Collection includes slide with L. tetrastei. Therefore, we can confirm that this is a valid host-louse association for Slovakia.

\section{Lipeurus maculosus Clay, 1938}

Host: Phasianus colchicus Linnaeus, 1758.

Ref.: Balát (1956, 1977); Straka (1987); Goldová et al. (2006).

Locations: Bratislava - Rača, 31 Dec. 1950 (Balát Coll., MMBC slide number 567); Bratislava, 10 Nov. 1948 and 3 Dec. 1950; Trnava, 22 Nov. 1950; Gabčíkovo, 21 Oct. 1954 (Balát 1956); Martin, 7 Dec. 1977; Nové Zámky, 1 Apr. 1978 (Straka Coll., AKMM); Game Management Centre, Rozhanovce, 2000-2004 (Goldová et al. 2006). 


\section{Lipeurus numidae (Denny, 1842)}

Host: Numida meleagris (Linnaeus, 1758)—captive bird.

Ref.: Straka (1987).

Location: Martin, 3 Apr. 1979 (Straka Coll., AKMM).

\section{Lipeurus pavo Clay, 1938}

Host: Pavo cristatus Linnaeus, 1758 - captive bird.

Ref.: Straka (1987).

Location: Martin, 19 Apr. 1979 (Straka Coll., AKMM—not present in the collection).

\section{Lunaceps numenii (Denny, 1842)}

Host: Numenius arquata (Linnaeus, 1758).

Ref.: Balát (1953, 1956, 1977).

Locations: Malacky, 5 Jun. 1950; Senné, 16 Apr. 1950 (Balát Coll., MMBC slide numbers 92, 458, 501); Zohor, 16 May 1948 (Balát 1956).

\section{Maculinirmus mundus (Nitzsch [in Giebel], 1866)}

Host: Oriolus oriolus (Linnaeus, 1758).

Ref.: Balát (1953, 1955b, 1956, 1977) as Brueelia munda.

Location: Járok u Nitry, 16 Jun. 1953.

Note: This species was placed in the genus Brueelia Kéler, 1936, but Gustafsson \& Bush (2017: 125) resurrected the genus Maculinirmus Złotorzycka, 1964a.

\section{Meropoecus meropis (Denny, 1842)}

Host: Merops apiaster Linnaeus, 1758.

Ref.: Balát (1956, 1977); Krištofík et al. (1996); this paper.

Locations: Radošovce-Vieska, 25 Jul. 1949; Sered', 4 Sep. 1948 (Balát Coll., MMBC slide numbers 215, 452, 454); Chotín, Mudroňovo, Jurský Chlm, Malá nad Hronom, Pavlová, Sikenička, May-Jul. 1995 for all locations (Krištofík et al. 1996); Virt, 19 Jul. 1998; Orechová potôň, 15 Aug. 2002 (Krištofík Coll., VETUNI).

\section{Meropsiella apiastri (Denny, 1842)}

Host: Merops apiaster Linnaeus, 1758.

Ref.: Balát (1953, 1956, 1977) as Brueelia apiastri; Gustafsson \& Bush (2017); this paper.

Locations: Radošovce—Vieska, 25 Jul. 1949; Sered', 4 Sep. 1948 (Balát Coll., MMBC slide number 214, 452); Orechová potôň, 15 Aug. 2002 (Krištofík Coll., VETUNI).

\section{Mulcticula hypoleucus (Denny, 1842)}

Host: Caprimulgus europaeus Linnaeus, 1758.

Ref.: Straka (1987).

Location: Blatnica, 5 Aug. 1982 (Straka Coll., AKMM).

\section{Neophilopterus incompletus (Denny, 1842)}

Host: Ciconia ciconia (Linnaeus, 1758).

Ref.: Balát (1977); Straka (1987); this paper.

Locations: Veškovce, 5 Aug. 1959 (Balát Coll., MMBC slide number 1158); Martin, 17-18 Feb. 1977; Socovce, 30 Apr. 1980; Krpel’any, 3 Sep. 1982 (Straka Coll., AKMM); Nové Zámky, 17 Sep. 1930 (Pfleger Coll., SNMB, NMPC).

\section{Neophilopterus tricolor (Burmeister, 1838)}

Host: Ciconia nigra (Linnaeus, 1758).

Ref.: Straka (1987); this paper.

Locations: Martin, 17 Feb. 1977 (Straka Coll., AKMM); Moravský Svätý Ján, 12 Oct. 1927 (Pfleger Coll., SNMB, NMPC). 
Olivinirmus glandarii (Denny, 1842)

Host: Garrulus glandarius (Linnaeus, 1758).

Ref.: Balát (1953) as Corvonirmus glandarii; Balát (1956, 1977); Straka (1987) as Brueelia (Corvonirmus) glandarii.

Locations: Járok u Nitry, 17 Jun. 1953; Slovakia, 12 Nov. 1948 (Balát Coll., MMBC slide numbers 358, 1132); Ťarchová pri Žit., 21 May 1950; Jablonica, 18 Mar. 1951; Podunajské Biskupice, 27 Oct. 1955 (Balát 1956); Martin, 10 Feb. 1978); Blatnica, 29 Mar. 1978 (Straka Coll., AKMM).

Note: This species was placed in the genus Brueelia Kéler, 1936, but Gustafsson \& Bush (2017: 199) resurrected the genus Olivinirmus Złotorzycka, 1964a.

Ornithobius bucephalus (Giebel, 1874)

Host: Cygnus olor (Gmelin, 1789).

Ref.: this paper.

Location: Dolný Štál, 13 Apr. 1997 (Krištofík Coll., VETUNI).

Note: This is the first record of Ornithobius bucephalus from Slovakia.

Otidoecus turmalis (Denny, 1842)

Host: Otis tarda Linnaeus, 1758.

Ref.: Balát (1953) as Otilipeurus turmale; Balát (1956, 1977); this paper.

Locations: Dunajská Streda, 23 Apr. 1949 (Balát Coll., MMBC slide number 550); Parkaň, 1 Jun. 1934 (Pfleger Coll., SNMB).

Note: The year of collection recorded by Balát (1956) and in his handwritten notes is "1948", but the label on his slide 550 reads " 1949 ".

\section{Oxylipeurus colchicus Clay, 1938}

Host: Phasianus colchicus Linnaeus, 1758.

Ref.: Straka (1987).

Location: Martin, 6 Jan. 1977 (Straka Coll., AKMM).

Oxylipeurus mesopelios (Nitzsch [in Giebel], 1866)

Host: Chrysolophus pictus (Linnaeus, 1758) — captive bird.

Ref.: Straka (1987).

Location: Martin, 5 May 1982 (Straka Coll., AKMM).

\section{Oxylipeurus tetraonis (Grube, 1851)}

Host: Tetrao urogallus Linnaeus, 1758.

Ref.: Balát (1953, 1956, 1977); Straka (1987).

Locations: Brezno, 20 Apr. 1949; Čierny Balog, 9 May 1956 (Balát Coll., MMBC slide numbers 1324, 1325); Brezno, 17 Apr. 1949 (Balát 1956); Oravská priehrada pri Námestove, 1 May 1977; Sučany, 2 May 1977; Ratkovo, 6 May 1977; Martin, 29 Oct. 1981 (Straka Coll., AKMM).

Notes: Straka (1987) recorded Lyrurus tetrix and a hybrid between T. urogallus and Lyrurus tetrix as hosts of Oxylipeurus tetraonis. It is known that T. urogallus and $L$. tetrix can produce hybrids in wild populations (Kleven et al. 2020), so finding lice on such a hybrid is both unusual and interesting. However, considering that Lyrurus tetrix is a natural host of Oxylipeurus minor (Złotorzycka, 1966) (Price et al. 2003: 204), the association between Oxylipeurus tetraonis and L. tetrix is most likely the result of a contamination or straggling.

\section{Pectinopygus gyricornis (Denny, 1842)}

Host: Phalacrocorax carbo (Linnaeus, 1758).

Ref.: Balát (1953) as Philichthyophaga longicornis (Piaget, 1880); Balát (1956, 1977); this paper.

Locations: Podunajské Biskupice, 13 Apr. 1951; Slovakia, May 1948 (Balát Coll., MMBC slide numbers 89, 612); Šamorín, 12-17 Sep. 1935 (Pfleger Coll., SNMB, NMPC). 


\section{Penenirmus albiventris (Scopoli, 1763)}

Host: Troglodytes troglodytes (Linnaeus, 1758).

Ref.: Balát (1953, 1955a, 1956, 1977); Sychra et al. (2014).

Locations: Žiar nad Hronom, formerly Svätý Kríž nad Hronom—dolina Kl’ak, 16 Apr. 1953; Ihráč, 19 Apr. 1953; Járok u Nitry, 13 Oct. 1953; Žarnovica-Rychňava, 21 Jun. 1953; Sklené Teplice, 10 Oct. 1953, 18 Apr. 1953 (Balát Coll., MMBC slide numbers 674-2x, 690-2x; 801, 803, 805, 806); Starý Smokovec, 14 Jun. 1955; Sklené Teplice, 17 Apr. 1953; Ihráč, 18 Apr. 1953 (Balát 1955a, 1956); Gbelce, 13 Apr. 2008 (Sychra et al. 2014).

Note: Balát (1956) incorrectly recorded the date of this collection as "Járok u Nitry (13 Oct. 1943)".

\section{Penenirmus auritus (Scopoli, 1763)}

Host: Dendrocopos leucotos (Bechstein, 1802).

Ref.: Balát (1956) as Penenirmus sp.; Balát (1977).

Location: Sološnica, 9 May 1948 (Balát Coll., MMBC slide number 351).

\section{Penenirmus auritus (Scopoli, 1763)}

Host: Dendrocopos major (Linnaeus, 1758).

Ref.: Balát (1953) as Philopterus auritus; Balát (1956, 1977); Straka (1987).

Locations: Sklené Teplice, 7 Oct. 1953 (Balát Coll., MMBC slide number 1065-not present in the collection); Sklené Teplice, 20 Apr. 1953; Gabčíkovo, 16 Mar. 1954; Rohovce, 16 Mar. 1955 (Balát 1956); Kláštor pod Znievom, 25 Jan. 1983 (Straka Coll., AKMM).

\section{Penenirmus auritus (Scopoli, 1763)}

Host: Dendrocopos syriacus (Hemprich \& Ehrenberg, 1833).

Ref.: Balát $(1956,1977)$ as Penenirmus peusi Eichler, 1953a; this paper.

Locations: Voderady, 10 Mar. 1953; Gabčíkovo, 22 Jul. 1953, 21 Mar. \& 21 Oct. 1954 (Balát 1956); Gbelce, 30 Apr.-1 May 2009 (VETUNI).

\section{Penenirmus auritus (Scopoli, 1763)}

Host: Dryobates minor (Linnaeus, 1758).

Ref.: Balát (1956) as Penenirmus sp.; Balát (1977); Straka (1987) as Penenirmus sp.

Locations: Gabčíkovo, 19 Mar. 1954 (Balát 1956); Slovany, 22 Nov. 1983 (Straka Coll., AKMM); Malženice, 11 Nov. 2006 (Krištofík Coll., VETUNI).

\section{Penenirmus gulosus (Nitzsch 1866)}

Host: Certhia familiaris Linnaeus, 1758.

Ref.: Balát (1956, 1977); Straka (1987).

Locations: Rohožník, Malé Karpaty, 16 Dec. 1951 (Balát Coll., MMBC slide number 649); Vrícko, 15 Nov. 1982 (Straka Coll., AKMM).

\section{Penenirmus heteroscelis (Nitzsch, 1866)}

Host: Dryocopus martius (Linnaeus, 1758).

Ref.: this paper.

Location: Bratislava, 7 Jun. 2010 (Krištofík Coll., VETUNI).

Note: This is the first record of Penenirmus heteroscelis from Slovakia.

\section{Penenirmus nirmoideus (Nitzsch [in Giebel], 1874)}

Host: Saxicola rubetra (Linnaeus, 1758).

Ref.: Balát (1956, 1977).

Locations: Nízke Tatry—Čertovica, 1 Jul. 1960; Plavecké Podhradie, 29 Apr. 1951; Velké Leváre—NPR Abrod, 11 May 1974, 18 May 1974 (Balát Coll., MMBC slide numbers 1379, 1452; 673, 1246, 1472). 


\section{Penenirmus pari (Denny, 1842)}

Host: Aegithalos caudatus (Linnaeus, 1758).

Ref.: Balát (1953, 1956, 1977).

Locations: Holíč, 27 Mar. 1948 (Balát Coll., MMBC slide numbers 3, 4); Sklené Teplice, 21 Apr. 1953; Banská Štiavnica_Počúvadla, 23 Apr. 1953 (Balát 1956).

\section{Penenirmus pici (J.C. Fabricius, 1798)}

Host: Picus canus Gmelin, 1788.

Ref.: Balát (1956, 1977).

Locations: Podunajské Biskupice, 20 Jul. 1953; Gabčíkovo, 21 Mar. 1954 (Balát 1956).

Penenirmus pici (J.C. Fabricius, 1798)

Host: Picus viridis Linnaeus, 1758.

Ref.: Balát (1956, 1977).

Locations: Bratislava, 10 Oct. 1948 (Balát Coll., MMBC slide number 353); Bratislava, 11 Nov. 1951 (Balát 1956).

Penenirmus visendus (Zlotorzycka, 1964a)

Host: Panurus biarmicus (Linnaeus, 1758).

Ref.: this paper.

Location: Gbelce, 14 Apr.-3 May 2008, 18 Apr.-1 May 2009, 17-21 Apr. 2016, 8-11 Jul. \& 1-2 Oct. 2019 (VETUNI)

Note: This is the first record of Penenirmus visendus from Slovakia.

\section{Philopterus acrocephalus Carriker, 1949}

Host: Acrocephalus melanopogon (Temminck, 1823).

Ref.: Najer et al. (2020).

Location: Gbelce, 13-30 Apr. 2008, 18 Apr.-1 May 2009, 18-20 Apr. 2016, 17 Apr. \& 9 Jul. 2019 (VETUNI; slides SK21-25 MMBC).

\section{Philopterus alexanderkoenigi (Eichler, 1953b)}

Host: Galerida cristata (Linnaeus, 1758).

Ref.: this paper.

Location: Štúrovo, 7 Jun. 1999 (Krištofík Coll., VETUNI).

Note: This is the first record of Philopterus alexanderkoenigi from Slovakia.

\section{Philopterus atratus (Nitzsch, 1818)}

Host: Corvus frugilegus Linnaeus, 1758.

Ref.: Balát (1953) as Philopterus corvi (Linnaeus, 1758); Balát (1956, 1977); Straka (1987); this paper.

Locations: Rohovce, 16 Mar. 1955, 19 May 1948; Súrovce, 21 May 1950 (Balát Coll., MMBC slide numbers 35, 524, 781); Bratislava, 20 Feb. 1952 (Balát 1956); Martin, 3 Jan. 1977; Blatnica, 9 Mar. 1977; Ďanová, 1 Nov. 1977; Stará Bystrica, 5 Jan. 1982 (Straka Coll., AKMM — any slide from Ďanová, 1 Nov. 1977 is not present in the collection); Kravany, 9 Jun. 1998; Zlatná na Ostrove, 20 Jan. 2001 (Krištofík Coll., VETUNI).

Note: Balát (1956) recorded the date of this collection as "Rohovce (16 Mar. 1956)", but the correct date is 1955. Straka (1987) recorded the location as "Krpel'any (5 Jan. 1982)”, but it is most likely an error, because in Straka's note on label of an available slide the location is given as "Stará Bystrica".

\section{Philopterus bischoffi (Eichler, 1951b)}

Host: Turdus pilaris Linnaeus, 1758.

Ref.: Balát (1953, 1956) as Philopterus sp.; Balát (1977); Bush et al. (2018); this paper.

Locations: Bratislava_Petržalka, 9 Mar. 1952; Plavecké Podhradie, Jan. 1951; Plavecký Mikuláš, 31 Dec. 1950 (Balát Coll., MMBC slide numbers 565—not present in the collection, 592, 619); Tatranská Javorina, Jun.-Jul. 
2015 (Bush et al. 2018); Štrbské pleso, 25 Jan. 1938 (Pfleger Coll., NMPC); Vrútky, 17 Apr. 1979; Blatnica, 1 Dec. 1980 (Straka Coll., AKMM).

\section{Philopterus citrinellae (Schrank, 1776)}

Host: Emberiza citrinella Linnaeus, 1758.

Ref.: Balát (1953) as "Philopterus subflavescens"; Balát $(1956,1977)$ as Philopterus citrinellae citrinellae; Straka (1987); this paper.

Locations: Košice, 29 Oct. 1953; Sklené Teplice, 14 Apr. 1953; Svätojurský Šúr, 13 Feb. 1951 (Balát Coll., MMBC slide numbers 1099, 1475; 1102—not present in the collection, SNMB slide number 645); Banská Štiavnica— Počúvadla, 23 Apr. \& 21 Jun. 1953; Járok u Nitry, 16 Apr. 1953; Vtáčnik, 19 Jun. 1953; Podunajské Biskupice, 21 Jul. 1953; Šaca, 4 Nov. 1953; Gabčíkovo, 15-24 Mar. \& 6 May 1954; Humenné, 9 May 1954 (Balát 1956); Kláštor pod Znievom, 9 Feb. 1981; ŠPR Kláštorské lúky pri Kláštore pod Znievom, 4 Nov. 1982; Vädžer, 2 Mar. 1983; Vrícko, 20 Mar. 1983 (Straka Coll., AKMM); Pataš, 28 Apr. 1999; Čunovo, 26 Jul. 1999 and 26 Jun. 2001 (Krištofík Coll., VETUNI).

Note: Straka (1987) recorded data on collections of Philopterus citrinellae from Emberiza citrinella and E. schoeniclus, but without mentioning which locality referred to each host species. Here we mention locations separately according to Straka's notes on relevant slides. Straka (1987) recorded one of the location and date for this host as "Blatnica, 11 Aug. 1977", but it is most likely an error, because in Straka's note on label of an available slide the host from this location and date is given as "Pyrrhula pyrrhula" (see below). Straka (1987) recorded the dates of collections as "Kláštor pod Znievom, 9 Feb. 1982" and "Vrícko, 20 Feb. 1983" but, according to Straka's notes on labels of relevant slides, the correct dates are probably "Kláštor pod Znievom, 9 Feb. 1981" and "Vrícko, 20 Mar. 1983".

\section{Philopterus citrinellae (Schrank, 1776)}

Host: Emberiza schoeniclus (Linnaeus, 1758).

Ref.: Balát (1953) as "Philopterus subflavescens"; Balát (1956) as P. citrinellae citrinellae; Balát (1977) as Philopterus residuus (Złotorzycka, 1964b); Straka (1987); this paper.

Locations: Senné, 16 Apr. 1950 (Balát Coll., MMBC slide number 503, SNMB slide number 572); Gabčíkovo, 22 Mar. 1954 (Balát 1956); Kláštor pod Znievom, 13 Mar. 1981; ŠPR Kláštorské lúky pri Kláštore pod Znievom, 25 Apr. 1978, 18 Mar. 1981, 10 May 1982; (Straka Coll., AKMM); Gbelce, 10 Jul. 2019 (VETUNI).

Note: Straka (1987) recorded data on collections of Philopterus citrinellae from Emberiza citrinella and E. schoeniclus, but without mentioning which locality referred to each host species. Here we mention locations separately according to Straka's notes on relevant slides. Straka (1987) recorded one of the location and date for this host as "Blatnica, 11 Aug. 1977", but it is most likely an error, because in Straka's note on label of an available slide the host from this location and date is given as "Pyrrhula pyrrhula" (see below).

\section{Philopterus citrinellae (Schrank, 1776)}

Host: Chloris chloris (Linnaeus, 1758).

Ref.: Balát $(1956,1977)$ as Philopterus citrinellae citrinellae; Hudec (1983) as Docophorulus chloridis (Schrank, 1776); this paper.

Locations: Gabčíkovo, 25 Mar. 1954 (Balát Coll., MMBC slide number 676); Sklené Teplice, 20 Apr. 1953 (Balát 1956); Gbelce, 19 Apr.-2 May 2008, 20 Apr. 2009 (VETUNI).

\section{Philopterus citrinellae (Schrank, 1776)}

Host: Pyrrhula pyrrhula (Linnaeus, 1758).

Ref.: Balát (1953) as "Philopterus subflavescens"; Balát $(1956,1977)$ as Philopterus citrinellae citrinellae; Hudec (1983) as Docophorulus pyrrhulae (Schrank, 1776); this paper.

Locations: Starý Smokovec, 11 Jun. 1955 (Balát 1956); Blatnica, 11 Aug. 1977; Kláštor pod Znievom, 4 Apr. 1979 (Straka Coll., AKMM); Bratislava-Mudroňova, 16 Jan. 2011 (Krištofík Coll., VETUNI).

Note: Although Straka (1987) listed "Blatnica, 11 Aug. 1977" as one the location and date for P. citrinellae, he probably mistakenly omitted P. pyrrhula as one of host. 
Philopterus citrinellae (Schrank, 1776)

Host: Spinus spinus (Linnaeus, 1758)

Ref.: Bush et al. (2018) as Philopterus sp.

Location: Tatranská Javorina, Jun.-Jul. 2015 (Bush et al. 2018).

\section{Philopterus coarctatus (Scopoli, 1763)}

Host: Lanius collurio Linnaeus, 1758.

Ref.: Balát (1953) as "Philopterus subflavescens"; Balát (1956, 1977) as P. coarctatus coarctatus; Straka (1987).

Locations: Gabčíkovo, 9 Sep. 1949, 22 Jul. 1953; Járok u Nitry, 16 Jun. 1953; Vtáčnik, 19 Jun. 1953; Žarnovica, 20 Jun. 1953; Kečovo, 27 Jul. 1954 (Balát 1956); Martin, 16 May 1977 (Straka Coll., AKMM—not present in the collection).

\section{Philopterus coarctatus (Scopoli, 1763)}

Host: Lanius excubitor Linnaeus, 1758.

Ref.: Balát (1953) as "Philopterus subflavescens"; Balát (1956, 1977) as Philopterus coarctatus fuscicollis (Burmeister, 1838); Szczykutowicz et al. (2006) as Docophorulus coarctatus; this paper.

Locations: Rohožník, Malé Karpaty, 5 Dec. 1951 (Balát Coll., MMBC slide number 607a, SNMB slide numbers 607b, c); Bardejov, 29 Feb. 1950; Šaca, 30 Oct. 1953; Šamorín, 16 Mar. 1955; Podunajské Biskupice, 26 Oct. 1955 (Balát 1956); NE Slovakia (Szczykutowicz et al. 2006); Andrejová, 9 Oct. 1963; Bardejov, 15 Dec. 1963 ; Chmel'ová, 5 Jun. 1964; Dubová, 28 May 1963, 2 Nov. 1963; Fulianka, 15 Mar. 1964; Gaboltov, 24 Jul. 1963 , 13 Sep. 1963; Hlinné, 1964; Hniezdne, 25 Mar. 1964; Kl’ušov, 36 Mar. 1964; Kolbovce, 16 Nov. 1963; Komarov, 19 Mar. 1964; Kružlová, 17 and 23 Jun. 1964; Kurima, 20 Sep. 1963; Kurimka, 28 May 1963; Ladomirova, 6 Nov. 1963; Lukavica, 28 Feb. 1964; Ol’šavce, 30 Mar. 1964; Plávnica pri Poprade, 16 Jul. 1964; Raslavice, 11 Oct. 1963, 10 Nov. 1963, 11 Sep. 1964; Roztoky, 28 and 31 May 1963; Smilno, 3 Jan. 1964, 6-7 Aug. 1964,12 Sep. 1964; Sveržov, 31 Mar. 1964; Tarnov, 20 Jun. 1963; Vyšný Orlík, 6 Nov. 1963; Vyšná Vol’a, 20 Jul. 1963, 17 Nov. 1963 (Weisz Coll., VETUNI).

Note: Balát (1956) recorded incorrectly the date of collection as "Rohožník, Malé Karpaty (5 Nov. 1951)".

\section{Philopterus coarctatus (Scopoli, 1763)}

Host: Lanius minor Gmelin, 1788.

Ref.: Balát (1977) as Philopterus coarctatus coarctatus.

Location: Zemplínska Široká—Rebrín, 4 Aug. 1959, 6 Aug. 1959 (Balát Coll., MMBC slide numbers 1490, 1493— not present in the collection).

Philopterus corvi (Linnaeus, 1758)

Host: Corvus corax Linnaeus, 1758.

Ref.: Straka (1987), this paper.

Location: Ďanová, 1 Sep. 1977, 1 Nov. 1977 (Straka Coll., AKMM).

\section{Philopterus crassipes (Burmeister, 1838)}

Host: Nucifraga caryocatactes (Linnaeus, 1758).

Ref.: Balát (1953, 1955a, 1956, 1977); Straka (1987) as Philopterus (Corvodocophorus) crassipes).

Locations: Starý Smokovec, 11 Jun. 1955 (Balát 1955a, 1956); Martin, 23 Mar. 1977, 15 Nov. 1980; Belá, 11 Sep. 1978 (Straka Coll., AKMM).

Note: "Corvodocophorus" was used as a subgenus by Balát (1955a) and Straka (1987) but, as there is no description, it is a nomen nudum.

\section{Philopterus curvirostrae (Schrank, 1776)}

Host: Loxia curvirostra Linnaeus, 1758.

Ref.: Balát (1953) as "Philopterus subflavescens"; Balát (1956, 1977) as Philopterus citrinellae curvirostrae.

Locations: Čachtice, 20 Mar. 1949; Tatranská Lomnica, 21 Mar. 1958 (Balát Coll., MMBC slide numbers 1426; 1431 - not present in the collection). 


\section{Philopterus emiliae Balát, 1955a}

Host: Prunella collaris (Scopoli, 1769).

Ref.: Balát (1955a, 1956, 1977); Straka (1987); Janiga \& Kubašková (2000); Janiga \& Mičková (2004).

Locations: Vysoké Tatry—Skalnaté Pleso, 15 Jun. 1955 (Balát Coll., MMBC slide numbers 698, 709; 707—not present in the collection); Lazany, 1 Feb. 1981 (Straka Coll., AKMM); Vysoké Tatry-Vysoké Tatry-Malá Studená dolina, Velická dolina, Skalnatá dolina, Pol'ský hrebeň, Rysy, Belianské Tatry, Batizovské pleso; Nízké Tatry—Ďumbier - dolina Štiavnica, Pol’ana, Chopok-Konské; Vel'ká Fatra—Malinô Brdo, 1988-2000 (Janiga \& Kubašková 2000; Janiga \& Mičková 2004).

\section{Philopterus excisus Nitzsch, 1818}

Host: Delichon urbicum (Linnaeus, 1758).

Ref.: Balát (1953, 1955a, 1956, 1977); Hudec (1983) as Cypseloecus excisus; Straka (1987); this paper.

Locations: Bojnice, 23 Apr. 1954; Bratislava, 14 Sep. 1948 (Balát Coll., MMBC slide numbers 191, 683); Nový Smokovec, 18 Jun. 1955 (Balát 1955a, 1956); Martin, 23 May 1978 (Straka Coll., AKMM); Dunajský Klátov, 2 Jun. 1998; Trávnik, 14 Jun. 1998 (Krištofík Coll., VETUNI).

\section{Philopterus fedorenkoae (Mey, 1983)}

Host: Acrocephalus arundinaceus (Linnaeus, 1758).

Ref.: Najer et al. (2020).

Location: Jakubov, 1 Sep. 1978 (Balát Coll., MMBC slide number 1501).

\section{Philopterus fortunatus (Zlotorzycka, 1964b)}

Host: Fringilla coelebs Linnaeus, 1758.

Ref.: Balát (1953) as "Philopterus subflavescens"; Balát (1956) as Philopterus sp.); Bush et al. (2018); this paper.

Locations: Žiar nad Hronom, formerly Svätý Kríž nad Hronom—dolina Klaak, 9 Oct. 1953 (Balát Coll., MMBC slide number 1104); Sklené Teplice, 20 Apr. 1953; Banská Štiavnica-Počúvadla, 23 Apr. \& 21 Jun. 1953; Podunajské Biskupice, 20 Jul.1953; Gabčíkovo, 22 Jul. 1953 and 19 Mar. 1954 (Balát 1956); Tatranská Javorina, Jun.-Jul. 2015 (Bush et al. 2018); Vel'ké Blahovo, 15 May 2010 (Krištofík Coll., VETUNI).

Note: Balát (1956) incorrectly recorded the dates of these collections as "Sklené Teplice (20 Apr. 1943) and "Banská Štiavnica—Počúvadla (23 Apr. \& 21 Jun. 1943)”.

\section{Philopterus fringillae (Scopoli, 1772)}

Host: Passer domesticus (Linnaeus, 1758).

Ref.: Balát (1953) as "Philopterus subflavescens"; Balát (1956, 1977); this paper.

Locations: Gabčíkovo, 22 Jul. 1953; Palín, 5 Apr. 1956; Sklené Teplice, 20 Apr. 1953 (Balát Coll., MMBC slide numbers 1060 — not present in the collection, 1129, 1317); Járok u Nitry, 17 Jun. 1953; Podunajské Biskupice, 21 Jul. 1953; Banská Štiavnica—Počúvadla, 23 Apr. 1953; Gabčíkovo, 16 Mar. 1954 (Balát 1956); Čunovo, 19 Jul. 2000; Láb, 28 Jun. 2001; Plavecký Štvrtok, 19 Jun. 2001 and 12 Jun. 2002 (Krištofík Coll., VETUNI).

Note: Balát (1956) incorrectly recorded the date of this collection as "Gabčíkovo (22 Apr. 1953)".

\section{Philopterus fringillae (Scopoli, 1772)}

Host: Passer montanus (Linnaeus, 1758).

Ref.: Balát (1956) as Philopterus sp.; Hudec (1983) as Docophorulus fringillae.

Locations: Gabčíkovo, 16 Mar. 1954; Hrhov, 31 Oct. 1953; Járok u Nitry, 16 Jun. 1953 (Balát Coll., MMBC slide numbers 967, 1019, 1025; 1476-not present in the collection); Gabčíkovo, 17 Mar. 1954 (Balát 1956); Hronovce, 13 Feb. 2001 (Krištofík Coll., VETUNI).

Note: Contrary to Price et al. (2003: 215), we agree with Macháček (1977) who regarded Philopterus montani (Złotorzycka, 1964b) — described from Passer montanus - as a junior synonym of Philopterus fringillae.

Philopterus fringillae (Scopoli, 1772)

Host: Pyrrhula pyrrhula (Linnaeus, 1758).

Ref.: Bush et al. (2018). 
Location: Tatranská Javorina, Jun.-Jul. 2015 (Bush et al. 2018).

Notes: Pyrrhula pyrrhula is parasitised by Philopterus citrinellae (see Price et al. 2003: 213; also see above). Considering that Bush et al. (2018: 44) did not discuss the unusual host-louse association they recorded for Philopterus fringillae, we can only speculate if their record is the result of a natural host switching or an contamination during or after collecting the lice.

\section{Philopterus garruli Boisduval \& Lacordaire, 1835}

Host: Garrulus glandarius (Linnaeus, 1758).

Ref.: Balát (1953, 1956, 1977); Straka (1987) as Philopterus (Corvodocophorus) garruli.

Locations: Slovakia, 12 Nov. 1948 (Balát Coll., MMBC slide number 358); Ťarchová pri Žit., 21 May 1950; Sklené Teplice, 24 Apr. 1953 (Balát 1956); Košt’any nad Turcom, 3 Dec. 1982; Martin, 10 Feb. 1978; Krpel'any, 5 Jan. 1982 (Straka Coll., AKMM).

Note: "Corvodocophorus" was used as a subgenus by Straka (1987) but, as there is no description, it is a nomen nudum.

\section{Philopterus gustafssoni Najer et al., 2020}

Host: Regulus regulus (Linnaeus, 1758).

Ref.: this paper.

Location: Kláštor pod Znievom, 2 Apr. 1979 (Straka Coll., AKMM).

Note: This is the first record of Philoperus gustafssoni from Slovakia.

\section{Philopterus guttatus (Denny, 1842)}

Host: Coloeus monedula (Linnaeus, 1758).

Ref.: Balát (1953, 1956, 1977).

Locations: Bratislava, 10 Oct. 1948; Pavlovce nad Uhom, 15 Apr. 1959, 9 Apr. 1956; Vojany, 20 Apr. 1959 (Balát Coll., MMBC slide numbers 352, 1165, 1168, 1186); Gabč́́kovo, 16-20 Mar. \& 19 Oct. 1954 (Balát 1956).

\section{Philopterus hanzaki Balát, 1955a}

Host: Anthus spinoletta (Linnaeus, 1758).

Ref.: Balát (1955a, 1956, 1977).

Location: Belianské Tatry—šafránová louka/pašienok pod Bujačím vrchom, 3 May 1952 (Balát Coll., MMBC slide number 702).

\section{Philopterus markevichi Fedorenko \& Volkov, 1977}

Host: Ficedula parva (Bechstein, 1792).

Ref.: Balát (1953) as "Philopterus subflavescens"; Balát (1956) as Philopterus sp.

Location: Trenčianské Teplice, 15 Jun. 1950 (Balát Coll., MMBC slide numbers 484-3x, 485).

\section{Philopterus microsomaticus Tandan, 1955}

Host: Hirundo rustica Linnaeus, 1758.

Ref.: Balát (1956) as Philopterus excisus Nitzsch, 1818; Hudec (1983) as Cypseloecus excisus microsomaticus; this paper.

Locations: Humenné, 12 Jun. 1954 (Balát 1956); Gbelce, 24-30 Apr. 2008, 27 Apr. 2009 (VETUNI).

\section{Philopterus microsomaticus Tandan, 1955}

Host: Riparia riparia (Linnaeus, 1758).

Ref.: Balát (1956) as Philopterus excisus Nitzsch, 1818; Hudec (1983) as Cypseloecus excisus subsp.; this paper. Locations: Humenné, 10 Jun. 1954 (Balát Coll., MMBC slide number 997); Gbelce, 28 Apr. 2008 (VETUNI).

Philopterus modularis (Denny, 1842)

Host: Prunella modularis (Linnaeus, 1758).

Ref.: Balát (1955a, 1956, 1977); Bush et al. (2018); Janiga (2018, 2019). 
Locations: Tatranská Javorina, 28 Jun. 1952 (Balát Coll., MMBC slide number 650 - not present in the collection); Tatranská Javorina, Jun.-Jul. 2015 (Bush et al. 2018); Vysoké Tatry, Nízké Tatry, Velká Fatra, Chočská vrchovina, 2007-2010 (Janiga 2018, 2019).

\section{Philopterus ocellatus (Scopoli, 1763)}

Host: Corvus cornix Linnaeus, 1758.

Ref.: Balát (1953, 1955a, 1956, 1977); Straka (1987) as Philopterus (Corvodocophorus) ocellatus.

Locations: Bojnice, 4 May 1953; Bratislava—Petržalka, 19 Feb. 1950; Banská Štiavnica—Počúvadla, 13 May 1958 (Balát Coll., MMBC slide numbers 525; 779, 1349); Tatranská Lomnica, 8 Jun. 1955; Bratislava—Petržalka, 5 Feb. 1949 and 4 Feb. 1950; Gabčíkovo, 20 Mar. 1954; Humenné, 5 Jun. 1954 (Balát 1955, 1956); Martin (27 Jan. 1977; Kláštor pod Znievom, 13 Mar. 1981 (Straka Coll., AKMM).

Note: "Corvodocophorus" was used as a subgenus by Balát (1955a) and Straka (1987) but, as there is no description, it is a nomen nudum.

\section{Philopterus ornatus (Nitzsch, 1866)}

Host: Oriolus oriolus (Linnaeus, 1758).

Ref.: Balát (1953, 1956, 1977).

Locations: Michalovce, May 1949 (Balát Coll., SNMB slide number 479); Plavecké Podhradie, 6 May 1951; Járok u Nitry, 16 Jun. 1953 (Balát 1956).

\section{Philopterus pallescens (Denny, 1842)}

Host: Poecile palustris (Linnaeus, 1758).

Ref.: Balát (1956, 1977); Mey (1988).

Location: Podunajské Biskupice, 27 Oct. 1955 (Balát Coll., MMBC slide number 1551).

Notes: We agree with Mey (1988) in that Philopterus pallescens only parasitises Poecile palustris (see note under Philopterus thuringiacus from Parus major).

\section{Philopterus passerinus (Denny, 1842)}

Host: Motacilla alba Linnaeus, 1758.

Ref.: Balát (1953) as "Philopterus subflavescens"; Balát (1956, 1977).

Locations: Sklené Teplice, 24 Apr. \& 17 Apr. 1953; Žarnovica—Rychňava, 21 Jun. 1953 (Balát 1956).

\section{Philopterus peripariphilus (Mey, 1988)}

Host: Periparus ater (Linnaeus, 1758).

Ref.: Hudec (1983) as Docophorulus sp.; Mey (1988) as Docophorulus hercynicus peripariphylus; this paper.

Location: Sklené Teplice, 24 Apr. 1953 (Balát Coll., MMBC slide number 1147).

Notes: Although the Balát Collection contains only three nymphs of Philopterus from Periparus ater, we follow Mey (1988) in that these specimens most likely belong to $P h$. peripariphilus.

\section{Philopterus picae (Denny, 1842)}

Host: Pica pica (Linnaeus, 1758).

Ref.: Balát (1953, 1956, 1977); Straka (1987) as Philopterus (Corvodocophorus) picae; this paper.

Locations: Gabčíkovo, 24 Mar. 1954 (Balát Coll., MMBC slide number 848-3x); Vel'ké Topol’níky, 14 Feb. 1951; Gabčíkovo, 16 Mar. 1954 (Balát 1956); Blatnica, 31 Jan. 1977, 12 May 1977, 24 Jan. 1978; Belá, 18 Apr. 1978 (Straka Coll., AKMM); Bratislava, 23 May 1999 (Krištofík Coll., VETUNI).

Note: "Corvodocophorus" was used as a subgenus by Straka (1987) but, as there is no description, it is a nomen nudum.

\section{Philopterus rapax (Zlotorzycka, 1964b)}

Host: Fringilla montifringilla Linnaeus, 1758.

Ref.: Balát (1956) as Philopterus sp.

Locations: Cabaj u Nitry, 15 Oct. 1953; Šaca, 4 Nov. 1953 (Balát 1956). 


\section{Philopterus sittae Fedorenko, 1978}

Host: Sitta europaea Linnaeus, 1758

Ref.: Balát (1956) as Philopterus sp.; this paper.

Locations: Sklené Teplice, 18 Apr. 1953 (Balát Coll., MMBC slide numbers 786-4x, 1149); Banská ŠtiavnicaPočúvadla, 23 Apr. \& 21 Jun. 1953; Gabčíkovo, 17-23 Mar. 1954; Podunajské Biskupice, 27 Oct. 1955 (Balát 1956); Svätý Jur, 20 Mar. 1998 (Krištofík Coll., VETUNI).

\section{Philopterus stadleri (Eichler, 1959)}

Host: Alauda arvensis Linnaeus, 1758.

Ref.: this paper.

Location: Kláštor pod Znievom, 13 Mar. 1981; ŠPR Kláštorské lúky pri Kláštore pod Znievom, 13 Mar. 1983 (Straka Coll., AKMM); Dunajská Lužná, 25 May1997 (Krištofík Coll., VETUNI).

Note: This is the first record of Philopterus stadleri from Slovakia.

\section{Philopterus thuringiacus (Mey, 1988)}

Host: Parus major Linnaeus, 1758.

Ref.: Balát (1953) as "Philopterus subflavescens"; Balát (1956, 1977) as Philopterus pallescens (Denny, 1842); Straka (1987) as Philopterus pallescens; Mey (1988).

Locations: Rohovce, 16 Mar. 1955; Sklené Teplice, 15 Apr. 1953 (Balát Coll., MMBC slide numbers 1052, 1553, 1554, 1555); Sklené Teplice, 7 Oct. 1953 (Balát 1956); Vrícko, 12 Aug. 1982, 15 Nov. 1982 (Straka Coll., AKMM - any slide from Vrícko, 12 Aug. 1982 is not present in the collection).

Notes: We agree with Mey (1988) in that Philopterus thuringiacus parasitises Parus major only. Hence, records of Ph. pallescens from P. major by Balát $(1956,1977)$ and Straka (1987) are most likely Ph. thuringiacus.

\section{Philopterus turdi (Denny, 1842)}

Host: Turdus merula Linnaeus, 1758.

Ref.: Balát (1953) as "Philopterus subflavescens"; Balát (1956) as Philopterus sp.; Balát (1977) as Philopterus merulae (Denny, 1842); Hudec (1983) as Docophorulus merulae; Straka (1987) as Philopterus sp.

Locations: Bratislava, 10 Feb. 1952 (Balát Coll., MMBC slide number 609); Kláštor pod Znievom, 2 Apr. 1979 (Straka Coll., AKMM - not present in the collection).

\section{Philopterus turdi (Denny, 1842)}

Host: Turdus philomelos Brehm, 1831.

Ref.: Balát $(1953,1956)$ as Philopterus sp.; Balát (1977).

Locations: Járok u Nitry, 16 Jun. 1953; Pavlovce nad Uhom, 9 Apr. 1956 (Balát Coll., MMBC slide numbers 760, 1205).

\section{Philopterus vernus (Zlotorzycka, 1964b)}

Host: Turdus viscivorus Linnaeus, 1758.

Ref.: Balát (1953, 1956) as Philopterus sp.; Balát (1977).

Locations: Žiar nad Hronom, formerly Svätý Kríž nad Hronom—dolina Kl'ak, 16 Apr. 1953; Jablonov, 3 Nov. 1953; Vel'ká Ida, 28 Oct. 1953 (Balát Coll., MMBC slide numbers 740; 764, 765 — not present in the collection); Sklené Teplice, 10 Oct. 1953 (Balát 1956).

Notes: Balát (1956) also recorded this location and date: "Sklené Teplice (16 Apr. 1953)" but, according to his notes, the correct location is probably "dolina Kl'ak".

\section{Philopterus sp.}

Host: Anthus trivialis (Linnaeus, 1758).

Ref.: Hudec (1983) as Docophorulus sp; this paper.

Location: Nízke Tatry—Čertovica, 29 Jun. 1960 (Balát Coll., MMBC slide number 1148).

Notes: According to Price et al. (2003: 217) Anthus trivialis is parasitised by Philopterus vultuosus (Złotorzycka, 1964b). However, we can not confirm this record to species level because the only specimen of Philopterus from this host available in the Balát Collection is in very poor condition. 


\section{Philopterus sp.}

Host: Turdus torquatus Linnaeus, 1758.

Ref.: Hudec (1983) as Docophorulus sp.; this paper.

Location: Nízke Tatry—Čertovica, 29 Jun. 1960 (Balát Coll., MMBC slide numbers 1212, 1213, 1214-4x).

Notes: According to Price et al. (2003: 215) Turdus torquatus is parasitised by Philopterus nativus Fedorenko, 1977. However, specimens of Philopterus from this host available in the Balát Collection are in such poor condition that cannot be identified to species.

\section{Philopterus sp.}

Host: Acrocephalus scirpaceus (Hermann, 1804).

Ref.: this paper.

Location: Gbelce, 24 Apr. 2008, 18 Apr. 2009 (VETUNI).

Notes: Mey (1977) recorded an undescribed species of Philopterus from Acrocephalus scirpaceus. Since we found only one nymph and one female on this host, we cannot exclude the possibility that these lice are contaminants from another bird handled at the same time. However, as we did not examine any known host of Philopterus while collecting these specimens, we believe the two lice are natural and regular parasites on A. scirpaceus.

\section{Philopterus sp.}

Host: Acrocephalus schoenobaenus (Linnaeus, 1758).

Ref.: this paper.

Location: Gbelce, 20 Apr. 2009 (VETUNI).

Notes: According to Price et al. (2003: 215), Acrocephalus schoenobaenus is parasitised by Philopterus necopinatus (Złotorzycka, 1964b). Since we found only one nymph on this host, we cannot exclude the possibility that it is a contaminant from another bird handled at the same time. However, as we did not examine any known host of Philopterus while collecting this specimen, we believe this louse is a natural and regular parasite on $A$. schoenobaenus.

\section{Philopterus sp.}

Host: Saxicola rubetra (Linnaeus, 1758).

Ref.: Hudec (1983); this paper.

Location: Leles, 5 Aug. 1959 (Balát Coll., MMBC slide number 1466).

\section{Philopterus sp.}

Host: Troglodytes troglodytes (Linnaeus, 1758).

Ref.: Hudec (1983) as Docophorulus sp.

Location: Žiar nad Hronom, formerly Svätý Kríž nad Hronom—dolina Kl'ak, 16 Apr. 1953 (Balát Coll., MMBC slide number 691 - not present in the collection).

Notes: According to Price et al. (2003: 217), Troglodytes troglodytes is parasitised by Philopterus troglodytis Fedorenko, 1986. However, as there are no specimens of Philopterus from this host in the Balát Collection, we cannot confirm the identity of this species.

\section{Picicola candidus (Nitzsch, 1866)}

Host: Picus canus Gmelin, 1788.

Ref.: Balát (1953, 1956, 1977).

Locations: Podunajské Biskupice, 20 Jul. 1953 (Balát Coll., MMBC slide number 1092); Gabčíkovo, 17-21 Mar. 1954 (Balát 1956).

Note: While Straka (1987) mentioned P. canus as host, there is Picus viridis as host on the label of available slide in the Straka collection. Similarly as in other similar cases we decided to follow note on the slide label (see below).

\section{Picicola candidus (Nitzsch, 1866)}

Host: Picus viridis Linnaeus, 1758. 
Ref.: Balát (1953, 1956, 1977) as Picicola contiguus Złotorzycka, 1965; Straka (1987).

Locations: Bratislava, 10 Oct. 1948; Košice, 4 Nov. 1953 (Balát Coll., MMBC slide numbers 353, 1095); Bratislava, 20 Dec. 1951; Žiar nad Hronom, formerly Svätý Kríž nad Hronom, 10 Oct. 1953; Gabčíkovo, 16 Mar. \& 21 Oct. 1954 (Balát 1956); Martin, 17 Nov. 1978 (Straka Coll., AKMM).

Note: While Straka (1987) mentioned P. canus as host, there is Picus viridis as host on the label of available slide in the Straka collection. Similarly as in other similar cases we decided to follow note on the slide label.

Picicola superciliosa (Nitzsch [in Giebel], 1866)

Host: Dendrocoptes medius (Linnaeus, 1758).

Ref.: Balát $(1956,1977)$ as Brueelia superciliosa.

Location: Banská Štiavnica—Počúvadla, 21 Jun. 1953 (Balát 1956).

Quadraceps anagrapsus (Nitzsch [in Giebel], 1866)

Host: Chlidonias leucopterus (Temminck, 1815).

Ref.: Balát (1956, 1977) as Quadraceps pagasti (Eichler, 1951c).

Location: Senné, 21 May 1951 (Balát 1956).

Quadraceps bicuspis (Nitzsch [in Giebel], 1874)

Host: Charadrius dubius Scopoli, 1786.

Ref.: Balát (1953, 1956, 1977).

Locations: Stropkov, 27 Jun. 1954; Šal'a, 29 Jun. 1954 (Balát 1956).

Quadraceps fissus (Burmeister, 1838)

Host: Charadrius hiaticula Linnaeus, 1758.

Ref.: Straka (1987).

Location: Kláštor pod Znievom, 17 Apr. 1981 (Straka Coll., AKMM).

Quadraceps furvus (Burmeister, 1838)

Host: Tringa erythropus (Pallas, 1764).

Ref.: Balát (1953, 1956, 1977).

Location: Senné, 20 Apr. 1950 (Balát Coll., MMBC slide number 456).

Quadraceps junceus (Scopoli, 1763)

Host: Vanellus vanellus (Linnaeus, 1758).

Ref.: Balát (1953, 1956, 1977).

Locations: Plavecké Podhradie, 29 Apr. 1951; Baka, 6 Oct. 1951; Turňa nad Bodvou, 2 Nov. 1953 (Balát 1956).

Quadraceps ochropi (Denny, 1842)

Host: Tringa ochropus Linnaeus, 1758.

Ref.: Balát (1953, 1956, 1977).

Locations: Bratislava, 27 Mar. 1951 (Balát Coll., MMBC slide number 1176); Trnava, 26 Apr. 1955 (Balát 1956).

Quadraceps phaeonotus (Nitzsch [in Giebel], 1866)

Host: Chlidonias niger (Linnaeus, 1758).

Ref.: Balát (1953) as Koeniginirmus phaeonotus; Balát $(1956,1977)$.

Locations: Kolárovo, formerly Guta, 5 May 1949 (Balát Coll., SNMB slide number 509); Senné, 21 May 1951 (Balát 1956).

Quadraceps punctatus punctatus (Burmeister, 1838)

Host: Chroicocephalus ridibundus (Linnaeus, 1766).

Ref.: Straka (1987) as Quadraceps (Koeniginirmus) punctatus; this paper.

Locations: Sučany, 26 Nov. 1979 (Straka Coll., AKMM); Gabčíkovo, 31 Jul. 1997 (Krištofík Coll., VETUNI). 
Quadraceps ravus (Kellogg, 1899)

Host: Actitis hypoleucos (Linnaeus, 1758).

Ref.: Balát (1953, 1955a, 1956, 1977) as Quadraceps subfuscus Blagoveshtchensky, 1948; Straka (1987) as Quadraceps subfuscus.

Locations: Vysoké Tatry_Javorová dolina, 12 May 1952 (Balát Coll., MMBC slide number 705-6x; SNMB slide number 705); ŠPR Kláštorské lúky pri Kláštore pod Znievom, 10 May 1982 (Straka Coll., AKMM).

\section{Quadraceps sellatus (Burmeister, 1838)}

Host: Sterna hirundo Linnaeus, 1758.

Ref.: Balát (1953) as Koeniginirmus sellatus; Balát (1956, 1977).

Location: Senné, 21 May 1951 (Balát 1956).

Rallicola (Rallicola) cuspidatus (Scopoli, 1763)

Host: Rallus aquaticus Linnaeus, 1758.

Ref.: this paper.

Location: Gbelce, 27 Apr. 2009 (VETUNI).

Note: This is the first record of Rallicola (Rallicola) cuspidatus from Slovakia.

Rallicola (Rallicola) fulicae (Denny, 1842)

Host: Fulica atra Linnaeus, 1758.

Ref.: Straka (1987); this paper.

Locations: Martin, 29 Mar. 1977 (Straka Coll., AKMM—not present in the collection); Bratislava—Kopáč, 19 Feb. 1997 (Krištofík Coll., VETUNI).

\section{Rallicola (Rallicola) minutus (Nitzsch [in Giebel], 1866)}

Host: Gallinula chloropus (Linnaeus, 1758).

Ref.: this paper.

Location: Martin, 6 Apr. 1982 (Straka Coll., AKMM).

Note: This is the first record of Rallicola (Rallicola) minutus from Slovakia.

Rhynonirmus helvolus (Burmeister, 1838)

Host: Scolopax rusticola Linnaeus, 1758.

Ref.: Balát (1953, 1956, 1977).

Location: Mariánka, Mar. 1951 (Balát 1956).

\section{Rostrinirmus carpodaci Balát, 1981a}

Host: Carpodacus erythrinus (Pallas, 1770).

Ref.: Balát (1981a).

Location: Bobrov (Oravská přehrada—Orava dam), 1 Jul. 1973 (Balát Coll., MMBC slide number 1390—not present in the collection).

Note: This species was placed in the genus Sturnidoecus Eichler, 1944, but Gustafsson \& Bush (2017: 263) resurrected the genus Rostrinirmus Złotorzycka, 1964a.

\section{Rostrinirmus hudeci Balát, 1981a}

Host: Parus major Linnaeus, 1758.

Ref.: Balát (1981a).

Location: Podunajské Biskupice, 21 Jul. 1953 (Balát Coll., MMBC slide number 804—not present in the collection).

Note: This species was placed in the genus Sturnidoecus Eichler, 1944, but Gustafsson \& Bush (2017: 263) resurrected the genus Rostrinirmus Złotorzycka, 1964a. 


\section{Rostrinirmus ruficeps (Nitzsch [in Giebel], 1866)}

Host: Passer montanus (Linnaeus, 1758).

Ref.: Balát (1953) as Penenirmus ruficeps; Balát $(1956,1977)$ as Sturnidoecus ruficeps; this paper.

Locations: Gabčíkovo, 22 Jul. 1953; Hrhov, 31 Oct. 1953; Járok u Nitry, 16-17 Jun. 1953; Podunajské Biskupice, 21 Jul. 1953 (Balát Coll., MMBC slide numbers 1019, 1027-2x; 1022, 1031); Gabčíkovo, 17-21 Mar. 1954 (Balát 1956); Búč, 29 Jun. 1997; Zohor, 28 Jun. 2001; Malacky-Vinohrádok, 27 May 2002 (Krištofík Coll., VETUNI); Gbelce, 10 Jul. 2019 (VETUNI).

Note: This species was placed in the genus Sturnidoecus Eichler, 1944, but Gustafsson \& Bush (2017: 263) resurrected the genus Rostrinirmus Złotorzycka, 1964a.

\section{Saemundssonia (Saemundssonia) integer (Nitzsch [in Giebel], 1866)}

Host: Grus grus (Linnaeus, 1758).

Ref.: Balát (1956, 1977).

Location: Senné, Spring of 1955 (Balát Coll., MMBC slide number 1004).

\section{Saemundssonia (Saemundssonia) lari (O. Fabricius, 1780)}

Host: Chroicocephalus ridibundus (Linnaeus, 1766).

Ref.: Balát (1953) as Saemundssonia gonothorax lari; Balát $(1956,1977)$ as Saemundssonia mülleri = Saemundssonia muelleri); Straka (1987) as $S$. mülleri; this paper.

Locations: Bratislava-Petržalka, 2 Nov. 1949 (Balát 1956); Sučany, 26.11. 1979; Kláštor pod Znievom, 19 Jan. 1984 (Straka Coll., AKMM); Jakubovské rybníky, 20 Apr. 1997; Čunovo, 24 Apr. 1997; Gabčíkovo, 31 Jul. 1997 (Krištofík Coll., VETUNI).

\section{Saemundssonia (Saemundssonia) lobaticeps (Giebel, 1874)}

Host: Chlidonias leucopterus (Temminck, 1815).

Ref.: Balát (1956, 1977).

Location: Senné, 21 May 1951 (Balát 1956).

Saemundssonia (Saemundssonia) sternae (Linnaeus, 1758)

Host: Sterna hirundo Linnaeus, 1758.

Ref.: Balát (1956, 1977).

Location: Senné, 21 May 1951 (Balát 1956).

\section{Saemundssonia (Saemundssonia) tringae (O. Fabricius, 1780)}

Host: Calidris pugnax (Linnaeus, 1758).

Ref.: this paper.

Location: Štrba, 21 Mar. 1936 (Pfleger Coll., SNMB).

Note: This is the first record of Saemundssonia (Saemundssonia) tringae from Slovakia.

\section{Saemundssonia (Saemundssonia) sp.}

Host: Larus argentatus Pontoppidan, 1763.

Ref.: Straka (1987).

Location: Sučany, 14 Nov. 1979 (Straka Coll., AKMM).

Notes: According to Price et al. (2003: 234), Larus argentatus is parasitised by Saemundssonia (Saemundssonia) lari (O. Fabricius, 1780) but, as we have not been able to check Straka's specimens, we leave this record at the genus level. The host of this record may be Larus cachinnans (see Discsussion, below)

\section{Strigiphilus barbatus (Osborn, 1902)}

Host: Asio otus (Linnaeus, 1758).

Ref.: Balát (1956) as Strigiphilus asionis (Eichler, 1949); Balát (1977); Straka (1987) as Strigiphilus asionis); this paper.

Locations: Komárno, 23 Apr. 1954; Podunajské Biskupice, 26 Oct. 1955 (Balát 1956); Lipovec, 2 Feb. 1977 (Straka Coll., AKMM); Dunajská Streda, 6 Dec. 1998; Dolný Štál, 16 Jun. 1999 (Krištofík Coll., VETUNI). 
Strigiphilus celebrachys (Denny, 1842)

Host: Bubo scandiacus (Linnaeus, 1758) — captive bird.

Ref.: this paper.

Location: Zoo Bojnice, 10 Nov. 1963 (Balát Coll., MMBC slide numbers 1405).

\section{Strigiphilus cursitans (Nitzsch [in Giebel], 1861)}

Host: Athene noctua (Scopoli, 1769).

Ref.: Balát (1953) as Neodocophorus athene (Mjöberg, 1910); Balát (1956, 1977); Straka (1987); this paper.

Locations: Plavecký Mikuláš, 14 Jan. 1951 (Balát Coll., MMBC slide number 583); Horný Kalník, 7 Feb. 1978 (Straka Coll., AKMM); Miloslavov, 16 May 2006 (Krištofík Coll., VETUNI).

Note: Straka (1987) recorded the location as "Horné Jaseno", but it is most likely an error, because in Straka's note on label of an available slide the location is given as "Horný Kalník".

\section{Strigiphilus cursor (Burmeister, 1838)}

Host: Asio flammeus (Pontoppidan, 1763).

Ref.: Balát (1956, 1977).

Locations: Cífer, 12 Oct. 1951, 18 Oct. 1951; Šal’a, 17 Sep. 1951; Vištuk, 7 Oct. 1951 (Balát Coll., MMBC slide numbers 626, 630); Voderady, 16 Sep. 1951; Slovenský Grob, 28 Oct. 1951; Rusovce, 18 Nov. 1951 (Balát 1956).

\section{Strigiphilus heterocerus (Grube, 1851)}

Host: Strix uralensis Pallas, 1771.

Ref.: Balát (1953) as Neodocophorus uralensis Eichler, 1949; Balát (1956, 1977).

Locations: Košice, 2 Dec. 1952; Kuzmice—okres Trebišov, 11 Nov. 1948; Snina, 16 Dec. 1948 (Balát Coll., MMBC slide numbers 212, 371, 790); Košice, 1 Dec. 1952; Medzilaborce, 11 Feb. 1956 (Balát 1956).

\section{Strigiphilus portigi Eichler, 1952}

Host: Strix aluco Linnaeus, 1758.

Ref.: Balát (1956, 1977); Straka (1987).

Locations: Myslenice, 19 Oct. 1952; Rožňava, 9 Feb. 1956 (Balát 1956); Vrútky, 17 Jan. 1978; Košt’any nad Turcom, 11 Oct. 1978 (Straka Coll., AKMM).

\section{Strigiphilus rostratus (Burmeister, 1838)}

Host: Tyto alba (Scopoli, 1769).

Ref.: Balát (1953, 1956, 1977); Hudec (1983) as Tytoniella rostrata; this paper.

Locations: Gabčíkovo, 13 Mar. 1951, 16 Sep. 1951 (Balát Coll., MMBC slide number 602, 624a, SNMB slide number 624b, c); Šafárikovo, 27 Dec. 1949; Krupina, 24 Mar. 1951 (Balát 1956); Sládkovičovo, 1 Dec. 2002 (Krištofík Coll., VETUNI).

\section{Strigiphilus strigis (Pontoppidan, 1763)}

Host: Bubo bubo (Linnaeus, 1758).

Ref.: Balát (1956, 1977).

Location: Šenkvice, formerly Čaníkovce, 19 Oct. 1952 (Balát Coll., MMBC slide number 797).

\section{Sturnidoecus sturni (Schrank, 1776)}

Host: Sturnus vulgaris Linnaeus, 1758.

Ref.: Balát (1956, 1977); this paper.

Locations: Banská Štiavnica—Počúvadla, 23 Apr. 1953; Kláštor pod Znievom, 10 May 1956 (Balát Coll., MMBC slide numbers 831, 1124); Gbelce, 13 Apr.-1 May 2008, 17 Apr. 2016 (VETUNI). 
Sturnidoecus tulackovae (Balát, 1981a)

Host: Locustella fluviatilis (Wolf, 1810).

Ref.: Balát (1981a).

Location: Senica, 5 Jun. 1971 (Balát Coll., MMBC slide numbers 1406, 1407-2x, 1408-2x, 1409).

\section{Turdinirmus merulensis (Denny, 1842)}

Host: Turdus merula Linnaeus, 1758.

Ref.: Balát (1977) as Brueelia merulensis; Hudec (1983) as Brueelia turdinirmus.

Location: Hronov, 17 Aug. 1953 (Balát Coll., MMBC slide number 763).

Note: This species was placed in the genus Brueelia Kéler, 1936, but Gustafsson \& Bush (2017: 117) resurrected the genus Turdinirmus Eichler, $1951 \mathrm{~b}$.

Upupicola upupae (Schrank, 1803)

Host: Upupa epops Linnaeus, 1758.

Ref.: Balát (1956, 1977).

Location: Šal'a, 29 Jun. 1954 (Balát 1956).

\section{Family Trichodectidae Kellogg, 1896a}

\section{Bovicola (Bovicola) alpinus Kéler, 1942}

Host: Rupicapra rupicapra (Linnaeus, 1758).

Ref.: Balát (1955a, 1956, 1977) as Damalinia alpina; Krištofík \& Danko (2012).

Location: Javorina, 18 Jun. 1955 (Balát Coll., MMBC slide number 700-2x).

\section{Bovicola (Bovicola) caprae (Gurlt, 1843)}

Host: Capra hircus Linnaeus, 1758.

Ref.: Balát (1956, 1977) as Damalinia caprae; Máca (1991).

Locations: Malé Trnie, 5 Apr. 1953 (Balát Coll., MMBC slide number 794-2x); Spišská Nová Ves, 11-12 Apr. 1989 (Máca 1991).

\section{Damalinia (Cervicola) meyeri (Taschenberg, 1882)}

Host: Capreolus capreolus (Linnaeus, 1758).

Ref.: Balát (1956, 1977); Máca (1991); Krištofík \& Danko (2012).

Locations: Sklené Teplice, 9 Oct. 1953 (Balát Coll., MMBC slide number 1377); Plavecký Mikuláš, Jul. 1952 (Balát 1956).

Notes: Balát (1956) recorded the date of the sample from Plavecký Mikuláš as "Jul. 1952”, but Máca (1991) recorded it as "3 Jun. 1951". However, we are unable to confirm which is the correct date, or if there are two different collection dates from the same locality.

Felicola (Felicola) subrostratus (Burmeister, 1838)

Host: Felis catus Linnaeus, 1758.

Ref.: Straka (1987).

Location: Kláštor pod Znievom, 6 May 1977 (Straka Coll., AKMM).

Stachiella ermineae Hopkins, 1941

Host: Mustela erminea Linnaeus, 1758.

Ref.: Krištofík \& Danko (2012); this paper.

Locations: Slovakia (Krištofík \& Danko 2012); Zohor, 11 Jun. 1998 (Krištofík Coll., VETUNI).

Stachiella jacobi Eichler, 1941b

Host: Mustela putorius Linnaeus, 1758. 
Ref.: Krištofík \& Danko (2012).

Location: Slovakia (Krištofík \& Danko 2012).

Stachiella mustelae (Schrank, 1803)

Host: Mustela nivalis Linnaeus, 1758.

Ref.: Straka (1982, 1987); Krištofík \& Danko (2012); this paper.

Locations: Muránska planina-Suché doly, 17 May 1979—-phoresis with fly Pollenia rudis from the family Polleniidae (Straka 1982); Martin, 25 Sep. 1980; Blatnica, 25 Jun. 1982 (Straka Coll., AKMM); Vysoká, 10 Apr. 1998; Studené, 19 Jun. 2005 (Krištofík Coll., VETUNI).

Note: Straka (1982) recorded a case of phoresis involving Stachiella mustelae on Pollenia rudis (J.C. Fabricius, 1794), the cluster fly.

Trichodectes melis (J.C. Fabricius, 1805)

Host: Meles meles (Linnaeus, 1758).

Ref.: Štefan (1977); Straka (1987); Lukáš et al. (1991, 1992); Krištofík \& Danko (2012).

Locations: Žiar nad Hronom (Štefan 1977); Kláštor pod Znievom, 31 Jul. 1977; Lipovec, 2 Aug. 1977; Martin, 3 Oct. 1977 (Straka Coll., AKMM); Nové Mesto nad Váhom—Turecký vrch, 20 Oct. 1982; Čachtice, 15 Aug. 1983; Nová Bošáca, 24 Oct. 1985; Devínska Kobyla, 27 Sep. 1986; Banská Štiavnica, 16 Sep. 1988; Porúbka, 2 Aug. 1991 (Lukáš et al. 1991, 1992).

\section{Discussion}

From a total of 365 species of birds recorded in Slovakia (including six non-natives), there are records of chewing lice from 171 (46.8\%) species (Table S3). The proportion is much lower in mammals, with only eight species (29.6\%) recorded with chewing lice from a total of 27 (Table S3). Chewing lice have been recorded mainly from birds breeding in Slovakia, especially perching birds (Passeriformes; Černecký et al. 2020), of which 70 species are listed here, representing 19.2\% of the total number of birds (Table 3). Most of 194 bird species without lice recorded from them in Slovakia are either migrants or rare, accidental visitors (Černecký et al. 2020; Table S5). Furthermore, there are no published reports of chewing lice anywhere in the world from 24 species of birds which occur in Slovakia (Table S5; Price et al. 2003).

As expected, higher proportions of bird species with recorded lice are from bird groups with smaller numbers of species, such as Bucerotiformes, Caprimulgiformes, Ciconiiformes, Coraciiformes, Galliformes and Piciformes, which have 1 to 10 members in Slovakia. The smaller proportions of recorded lice in other groups are affected by many factors, one being a large number of species in the group, e.g. 71 species of Charadriiformes in Slovakia, but only 14 species with recorded lice (Tables 5, S5); another important factor is collection effort, e.g. no lice have been recorded from common and widely distributed species, such as Mareca strepera, Coturnix coturnix, Serinus serinus, Luscinia megarhynchos, Phoenicurus ochruros, Phoenicurus phoenicurus, Sylvia atricapilla, Sylvia borin, Sylvia communis, Sylvia curruca, and only a small number of lice are reported from captive birds such as Gallus gallus. Chewing louse populations are not distributed uniformly across their host population (Clay 1949a; Gustafsson \& Zou 2020), so it is possible that louse populations may be low on certain hosts in Slovakia, in which case, more intensive sampling may be required. Also, host migration can have an impact on the number of ectoparasites (Literák et al. 2015).

In this paper, we cover a period of almost 100 years of research on chewing lice in Slovakia. During this period, the species composition of the Slovakian avifauna has changed (Černecký et al. 2020). There are new records for species of birds occurring in this region during the last decades (e.g. Šrank 2010; Kvetko \& FK SOS/BirdLife Slovensko 2017; SOS/BirdLife Slovakia 2020). Most of these species have not been examined for lice yet, including Emberiza pusilla, Iduna pallida, Phylloscopus proregulus and Phylloscopus schwarzi, which have no lice recorded from them worldwide (Table S5; Price et al. 2003). On the other hand, some species have become rare or have completely disappeared from Slovakia, e.g. Circaetus gallicus, Coracias garrulus and Otis tarda (see Černecký et al. 2020). Old reports of lice from these hosts are valuable, showing that ectoparasites may also have become extirpated in this area. While some species of lice from these hosts are well documented across Europe-e.g. Capraiella sub- 
cuspidata ex Coracias garrulus reported from Finland, Germany, Hungary, Italia, Poland, Romania and Spain (Mey 2021) - information on the geographic distribution of other louse species from the same hosts is limited, e.g. Meromenopon incisum (Giebel, 1866) ex Coracias garrulus is known only from Germany and Hungary (Mey 2021), Otidoecus turmalis (Denny, 1842) ex Otis tarda from Germany, Hungary and Spain (Mey 2021), and Degeeriella leucopleura (Nitzsch [in Giebel], 1874) ex Circaetus gallicus is known only from Spain (Pérez et al. 1996).

Changes in the taxonomy of the hosts can also affect numbers and types of host-louse associations. One good example is Corvus corone, traditionally divided in two subspecies, Corvus corone corone and Corvus corone cornix, but now recognised as two separate species (Gill et al. 2021). It can be assumed that both taxa would harbour the same species of lice, but at present all records from Slovakia are from Corvus cornix. Therefore, other species recorded from Corvus corone cannot be regarded as present in this country. Similarly, Larus cachinnans Pallas, 1811 was raised to species level (Liebers et al. 2001) from a subspecies of Larus argentatus; since Larus cachninnans is more common in Slovakia than Larus argentatus (see Černecký et al. 2020) we can speculate that the host of Saemundssonia (Saemundssonia) sp. reported by Straka (1987) as Larus argentatus was most likely Larus cachninnans. The same scenario can be applied to the lice of Anser fabalis reported by Balát (1956), because Central European geese traditionally identified as Anser fabalis are most likely Anser serrirostris Gould, 1852, a taxon recently separated from Anser fabalis (see Sangster \& Oreel 1996). More samples from hosts correctly identified are needed to know which lice parasitise these two goose species.

We regard the record of two specimens of Laemobothrion from Coracias garrulus reported by Balát (1956) as likely stragglers, because Coracias belongs to an order which is not known to harbour any species of Laemobothrion (Price et al. 2003: 309). However, we cannot completely exclude the possibility that they represent a natural host-louse association. The specimens are a third instar nymph and a teneral female, which differ from all Laemobothrion known from the area. Since examples of some well-established louse populations on unexpected hosts are known-i.e. Myrsidea imbricata (Neumann, 1891) on the hummingbird Chlorostilbon swainsonii; and Neopsittaconirmus inexpectatus Guimarães, 1974 on the pygmy falcon Polihierax semitorquatus - we believe that there could be a natural population of Laemobothrion on Coraciasgarrulus. Further collecting is necessary to confirm or disprove this hypothesis. Although C. garrulus is now very rare or even extirpated in Slovakia, it is still relatively abundant in other parts of its geographic distribution.

Considering that the territory of Slovakia is relative small, it is not surprising that most of the louse species recorded from this country were originally described elsewhere. However, nine new species were described based on material collected in Slovakia: Brueelia balati, Brueelia breueri, Brueelia rosickyi, Philopterus emiliae, Philopterus hanzaki, Philopterus thuringiacus, Rostrinirmus carpodaci, Rostrinirmus hudeci and Sturnidoecus tulackovae. Among these species, Ph. emiliae, R. carpodaci and S. tulackovae are still known from Slovakia only (Mey 2021).

To increase our knowledge of louse diversity as well as their host and geographic distributions in this country, further collecting and research are necessary, especially on hosts in the bird orders Charadriiformes and Passeriformes; these two orders together account to $60 \%$ of all the 194 bird taxa without records of lice in Slovakia (Table S3). Also, louse collecting from mammals is needed, considering that there are records of lice from only $29.6 \%$ of all the species living in Slovakia (Table S3).

\section{Acknowledgements}

We thank all our co-workers in the field and the students who helped us with the revision of Balát's and Pfleger's Collections. We specially thank Igor Malenovský (Moravian Museum Brno, Czech Republic), Vladimír Janský (Slovak National Museum, Bratislava, Slovakia), Pavel Chvojka (National Museum, Prague, Czech Republic), and Matej Žiak (Slovak National Museum in Martin, Andrej Kmet Museum, Slovakia) for enabling us to examine their collections. We are indebted to Ricardo Palma (Museum of New Zealand, Wellington, New Zealand) for his valuable advice and revision of the first draft of this paper, and to Terry D. Galloway (Department of Entomology, University of Manitoba, Winnipeg, Manitoba, Canada) for many helpful comments that improved this paper. We were supported by the project FVHE/Široký/ITA2021 from the University of Veterinary Sciences, Brno, Czech Republic. 


\section{Supplementary materials}

Supplementary data accompaning this paper is available from: https://doi.org/10.11646/zootaxa.5069.1.1

\section{References}

Adam, C. (2008) Studiul morfologic, sistematic, faunistic si al specificitatii parazitare la unele specii de malofage (Phthiraptera: Amblycera, Ischnocera) parazite pe pasari din Romania. [Morphological, systematic, faunistic and parasitological study of some species of chewing lice (Phthiraptera: Amblycera, Ischnocera) parasitising birds in Romania]. PhD Thesis, Universitatea din Bucuresti, Bucharest, 438 pp. [in Romanian]

Balát, F. (1952) K poznaniu druhov rodu Ricinus De Geer 1778 (Mallophaga). [Contribution to knowledge of species of the genus Ricinus De Geer 1778 (Mallophaga)]. Biologický sborník Slovenskej akademie vied a umení, 7, 155-170. [in Slovak]

Balát, F. (1953) Mallophaga zjištěná na ptácích Moravy a Slovenska. [Mallophaga found on birds from Moravia and Slovakia]. Spisy vydávané Prĭrodovédeckou fakultou Masarykovy university, M6, 348, 169-176. [in Czech]

Balát, F. (1955a) Všenky z Tatranského národního parku. [Chewing lice from Tatra national park]. Folia Zoologica et Entomologica, 4 (4), 389-398. [in Czech]

Balát, F. (1955b) Př́spěvek k poznání všenek rodu Brüelia I. [Contribution to the knowledge of chewing lice of the genus Brüelia I.]. Práce Brněnské Základny Československé Akademie Věd, 27, 499-524. [in Czech]

Balát, F. (1956) Přehled všenek (Mallophaga), zjištěných na ptácích a savcích Slovenska. [Overview of chewing lice (Mallophaga) from birds and mammals from Slovakia]. Sborník krajského múzea v Trnave, 2, 56-77. [in Czech]

Balát, F. (1958) Př́spěvek k poznání všenek bulharských ptáků [Beitrag zur Kenntnis der Mallophagenfauna der bulgarischen Vögel]. Práce Brněnské Základny Československé Akademie Věd, 30, 397-422. [in German]

Balát, F. (1966) Federlinge tschechoslowakischer Uferschwalben. Angewandte Parasitologie, 7 (1), 20-30. [in German]

Balát, F. (1977) Enumeratio insectorum Bohemoslovakiae: Mallophaga. Acta Faunistica Entomologica Musei Nationalis Prague, 15 (Supplementum 4), 45-52. [in German]

Balát, F. (1981a) New species of biting lice (Mallophaga) of the genera Penenirmus and Rostrinirmus. Folia Parasitologica, $28(2), 161-168$.

Balát, F. (1981b) A contribution to the knowledge of biting lice (Mallophaga) found on passerines (Passeriformes). Folia Parasitologica, 28 (3), 273-282.

Barbosa, A., Merino, S., de Lope, F. \& Møller, A.P. (2002) Effects of feather lice on flight behavior of male barn swallows. Auk, 119 (1), 213-216. https://doi.org/10.1093/auk/119.1.213

Bechet, I. (1963) Specii de Lagopoecus (Mallophaga) din fauna Republicii Populare Romîne. [Species of Lagopoecus (Mallophaga) from the fauna of the Romanian People's Republic.] Studii și cerccetări de biologie, Academia Republicii Populare Romine, Filiala Cluj, 14 (2), 257-263. [in Romanian]

Blagoveshtchensky, D.I. (1940) Mallophaga s ptic Talisa. [Mallophaga from the birds of Talysh.] Parazitologiceskij Sbornik, Moscow and Leningrad [ = Magasin de Parasitologie de l'institut Zoologique de l'Académie des Sciences de l'URSS ], 8, 25-90. [in Russian]

Blagoveshtchensky, D.I. (1948) Mallophaga s ptic barabinsckich ozer. (1). [Mallophaga from the birds of Lake Barabinsk. Part 1.] Parazitologiceskij Sbornik, Moscow and Leningrad [= Magasin de Parasitologie de l'institut Zoologique de l'Académie des Sciences de l'URSS ], 10, 259-294. [in Russian]

Blagoveshtchensky, D.I. (1951) [Mallophaga of Tadzhikistan.] Parazitologiceskij Sbornik, Moscow and Leningrad [= Magasin de Parasitologie de l'institut Zoologique de l'Académie des Sciences de l'URSS ], 13, 272-327 [in Russian]

Boisduval, J.-B.A.D. de \& Lacordaire, J.T. (1835) Second ordre. Anoploures. In: Boisduval, J.-B.A.D. de \& Lacordaire, J.T., Faune entomologique des environs de Paris; ou species général des insectes qui se trouvent dans un rayon de quinze a vingt lieues aux alentours de Paris. Vol. 1. Méquignon-Marvis, Père et Fils, Libraires, Paris, pp. 117-125. [in French]

Booth, D.T., Clayton, D.H. \& Block, B.A. (1993) Experimental demonstration of the energetic cost of parasitism in free-ranging hosts. Proceedings of the Royal Society of London, Series B-Biological Sciences, 253 (1337), 125-129. https://doi.org/10.1098/rspb.1993.0091

Brown, C.R., Brown, M.B. \& Rannala, B. (1995) Ectoparasites reduce long-term survival of their avian host. Proceedings of the Royal Society of London, Series B-Biological Sciences, 262 (1365), 313-319. https://doi.org/10.1098/rspb.1995.0211

Burmeister, H. (1838) Mallophaga. In: Burmeister, H., Handbuch der Entomologie. Vol. 2. No. 1. Enslin, Berlin, pp. $418-443$. [in German]

Bush, S.E., Harbison, C.W., Slager, D.L., Peterson, A.T., Price, R.D. \& Clayton, D.H. (2009) Geographic variation in the community structure of lice on western scrub-jays. Journal of Parasitology, 95, 10-13. https://doi.org/10.1645/GE-1591.1

Bush, S.E., Gustafsson, D.R. \& Clayton, D.H. (2018) New records of ectoparasites from passerine birds in the High Tatras of Slovakia. Oecologia Montana, 27, 43-45. 
Carriker, M.A, Jr (1949) On a collection of Mallophaga from Guam, Marianas Islands. Proceedings of the United States National Museum, 100 (3254), 1-24.

Carriker, M.A, Jr (1953) Neotropical Miscellany No. VI. New genus and species of Mallophaga. The Florida Entomologist, 36 (4), 151-160.

Carriker, M.A, Jr \& Shull, C.A. (1910) Some new species of Mallophaga from Michigan. Entomological News, 21 (2), $51-57$.

Černecký, J., Lešo, P., Ridzoň, J., Krištín, A., Karaska, D., Darolová, A., Fulín, M., Chavko, J., Bohuš, M., Krajniak, D., Ďuricová, V., Lešová, A., Čuláková, J., Saxa, A., Durkošová, J., Andráš, P. (2020) Stav ochrany vtáctva na Slovensku v rokoch 2013-2018. [Conservation status of birds in Slovakia in 2013-2018]. ŠOP SR, Banská Bystrica, 105 pp. [in Slovak]

Clay, T. (1938) Revision of the genera and species of Mallophaga occurring on Gallinaceous hosts.—Part I. Lipeurus and related genera. Proceedings of the Zoological Society of London, Series B, 108 (2), 109-204.

Clay, T. (1949a) Some problems in the evolution of a group of ectoparasites. Evolution, 3, 279-299.

Clay, T. (1949b) Systematic notes on the Piaget collections of Mallophaga.-Pt I. Annals and Magazine of Natural History, Series 12, 2, 895-921.

Clay, T. (1966) Contributions towards a revision of Myrsidea Waterston I. (Menoponidae: Mallophaga). Bulletin of the British Museum (Natural History) Entomology, 17, 327-395.

Clay, T. \& Meinertzhagen, R. (1941) Mallophaga miscellany.-No, 2. Annals and Magazine of Natural History, Series 11, 7, 329-346.

Clayton, D.H., Bush, S.E. \& Johnson, K.P. (2016) Coevolution of life on hosts: integrating ecology and history. University of Chicago Press, Chicago, 294 pp.

Conci, C. (1942) Il genere Myrsidea II. Una nuova specie di Myrsidea del Cinclus c. meridionalis Brehm. Annali del Museo Civico di Storia Naturale de Genova, 61, 287-289. [in Italian]

Cotgreave, P. \& Clayton, D.H. (1994) Comparative analysis of time spent grooming by birds in relation to parasite load. Behaviour, 131, 171-187.

De Geer, C. (1778) Des ricins. In: De Geer, C., Mémoires pour servir à l'histoire des insectes. Vol. 7. Pierre Hesselberg, Stockholm, pp. 69-82. [in French]

Denny, H. (1842) Monographia Anoplurorum Britanniae or, an essay on the British species of parasitic insects belonging to the order of Anoplura of Leach, with the modern divisions of the genera according to the views of Leach, Nitzsch, and Burmeister, with highly magnified figures of each species. Henry G. Bohn, London, xxiv $+262 \mathrm{pp}$.

Dik, B. \& Aydenizöz-Özkayhan, M. (2007) Mallophaga species on long-legged buzzards (Buteo rufinus) in Turkey. Türkiye Parazitoloji Dergisi, 31 (4), 298-301.

Durden, L. \& Musser, G.G. (1994) The sucking lice (Insecta, Anoplura) of the world: a taxonomic checklist with records of mammalian hosts and geographical distributions. Bulletin of the American Museum of Natural History, 218, 1-90.

Durrant, E.P. (1906) Descriptions of new Mallophaga. The Ohio Naturalist, 6 (7), 528-530.

Eichler, W. (1941a) Zur Klassifikation der Lauskerfe (Phthiraptera Haeckel: Rhynchophthirina, Mallophaga und Anoplura). Archiv für Naturgeschichte. Neue Folge, 10, 345-398. [in German]

Eichler, W. (1941b) Mallophagen-Synopsis. II. Genus Stachiella. Zoologischer Anzeiger, 136 (9-10), 187-189. [in German]

Eichler, W. (1943a) Mallophagen-Synopsis. IX. Genus Holomenopon. Mitteilungen der Münchener Entomologischen Gesellschaft, 33 (1), 236-239. [in German]

Eichler, W. (1943b) Mallophagen-Synopsis. VII. Genus Trinoton. Stettiner Entomologische Zeitung, 104, 96-101. [in German]

Eichler, W. (1943c) Mallophagen-Synopsis. IX. Genus Degeeriella. Zoologischer Anzeiger, 142, 92-93. [in German]

Eichler, W. (1946) Mallophagen-Synopsis. VIII. Genus Anatoecus. Tijdschrift voor Entomologie, 87, 74-76. [in German]

Eichler, W. (1947) Über einen seltenen Tauben-Federling, Bonomiella concii nov. spec. Tierärztliche Umschau, 2 (21-22), 264-265. [in German]

Eichler, W. (1948) Schutzfärbung bei Federlingen. Vögel der Heimat, 6, 103-108. [in German]

Eichler, W. (1949) Die Eulenfederlinge. Gruppen-Studien bei Mallophagen Nr. 5. Beiträge zur Taxonomischen Zoologie, 1, 7-22. [in German]

Eichler, W. (1950) Notulae Mallophagologicae. XIII. Neue Goniodidae. Doriana. Supplemento Annali del Museo Civico di Storia Naturale “G. Doria”, 1 (9), 1-8. [in German]

Eichler, W. (1951a) Notulae Mallophagologicae. XVII. Die Myrsideen. Zoologischer Anzeiger, 146, 45-53. [in German]

Eichler, W. (1951b) Die Federling der Drosseln. In: Eichler, W., Bedeutung der Vogelwelt in Forschung und Praxis. Vortrage der I. Ornithologische Tagung der DDR am 21-22 October 1950, Leipzig, pp. 29-47. [in German]

Eichler, W. (1951c) Mallophagen-Synopsis. XVII. Genus Koeniginirmus. Bonner Zoologische Beiträge, 2 (1-4), 125-134. [in German]

Eichler, W. (1952) Strigiphilus portigi nov. spec. (Malloph.). Der Zoologische Garten, Zeitschrift für gesamte Tiergärten, 19 $(2-4), 154$. [in German]

Eichler, W. (1953a) Mallophagen-Synopsis. XXIV. Genus Penenirmus (incl. Picophilopterus). Zoologischer Anzeiger, 150, 235-245. [in German]

Eichler, W. (1953b) Von Alexander Koenig gesammelte Federlinge. I. Von Singvogeln und Spechten. Bonner Zoologische Beiträge, 4 (3-4), 333-343. [in German]

Eichler, W. (1953c) Notulae Mallophagologicae. XII. Neue Menacanthinae. Beiträge zur Vogelkunde, 3 (3-4), 166-183. [in 
German]

Eichler, W. (1954a) Die Entwicklung der vordersten Saumborste bei Anaticola-Arten als Peitschenborste und zum fühlernanalogen Tastsinnesorgan. Zoologischer Anzeiger, 152, 32-35. [in German]

Eichler, W. (1954b) Deutsche Federlinge. I. Genus Brüelia. Naturwissenschaftliches Museum Nachrichten, 42, 59-66. [in Ger$\operatorname{man}]$

Eichler, W. (1959) Notizen zur angewandten Parasitenkunde (III). Das Deutsche Gesundheitswesen, 14, 1171-1175. [in German]

Eichler, W. \& Złotorzycka, J. (1975) Studien über Raubvogelfederlinge. VIII. Craspedorrhynchus fraterculus n. sp. von Aquila heliaca. Angewandte Parasitologie, 16, 153-161. [in German]

Emerson, K.C. (1949) Three new species of Mallophaga. Journal of the Kansas Entomological Society, 22 (2), $75-78$.

Emerson, K.C. (1972) Checklist of the Mallophaga of North America (north of Mexico). Part II. Suborder Amblycera. Deseret Test Center, Dugway Proving Ground, Dugway, Utah, 118 pp.

Fabricius, J.C. (1798) Pediculus. In: Fabricius, J.C., Supplementum Entomologiae Systematicae. Proft \& Storch, Hafniae, pp. 570-571. [in Latin]

Fabricius, J.C. (1805) Pediculus. In: Fabricius, J.C., Systema antliatorum secundum ordines, genera, species adiectis synonymis, locis, observationibus, descriptionibus. Carolus Reichard, Braunschweig, pp. 341-349. [in Latin]

Fabricius, O. (1780) Fauna Groenlandica systematice sistens animalia Groenlandiae occidentalis hactenus indagata. J.G. Rothe, Hafniae et Lipsiae, xvi +452 pp. [in Latin]

Fedorenko, I.A. (1975) [New species of biting lice from the genus Brüelia (Mallophaga: Ischnocera) in Passeriformes of the Ukraine]. Vestnik Zoologii, Kiev, 1975, 2, 46-51. [in Russian]

Fedorenko, I.A. (1977) [Materials on the Passeriformes biting lice in the Ukraine. Communication III. Philopterinae (Mallophaga, Ischnocera) Part 1]. Vestnik Zoologii, Kiev, 1977 (6), 33-38. [in Russian]

Fedorenko, I.A. (1978) [Materials on the Mallophaga fauna on Passeriformes of the Ukraine. Communication IV. Philopterinae (Mallophaga, Ischnocera) Part 2]. Vestnik Zoologii, Kiev, 1978, 2, 55-60. [in Russian]

Fedorenko, I.A. (1983) [Superfamily Menoponoidea. Fauna Ukrainy]. Institut Zoologii Akademii Nauk Ukraini, RSR, Kiev, 22 (5), 1-168 [in Russian]

Fedorenko, I.A. (1986) [New Philopterus species (Mallophaga: Philopteridae) — parasites of prunellid and troglodytid birds]. Vestnik Zoologii, Kiev, 1986, 2, 3-6. [in Russian]

Fedorenko, I.A. (1987) [Subfamily Philopterinae. Fauna Ukrainy]. Institut Zoologii Akademii Nauk Ukraini, RSR, Kiev, 22 (9), $1-166$ [in Russian]

Fedorenko \& Volkov (1977) On studying bird lice of the Philopterus genus parasitizing on flycatchers. Vestnik Zoologii, Kiev, 4, 60-70. [in Russian]

Gervais, F.L.P. (1844) Dicères épizoiques. In: Walckenaer, C.A. (Ed.), Histoire Naturelle des Insectes. Aptères. Tome 3. Librairie Encyclopédique de Roret, Paris, pp. 290-361. [in French]

Giebel, C.G.A. (1861) Die Federlinge der Raubvögel aus Chr. L. Nitzsch's handschriftlichem Nachlass zusammengestellt. Zeitschrift für die gesammten Naturwissenschaften, Halle, 17 (6), 515-529. [in German]

Giebel, C.G.A. (1866) Die im zoologischen Museum der Universität Halle aufgestellten Epizoen nebst Beobachtungen über dieselben. Zeitschrift für die gesammten Naturwissenschaften, Halle, 28 (11/12), 353-397. [in German]

Giebel, C.G.A. (1874) Insecta epizoa. Die auf Säugetieren und Vögeln schmarotzenden Insecten nach Chr. L. Nitzsch's Nachlass bearbeitet. Otto Wigand, Leipzig, xvi +308 pp. [in German]

Gill, F., Donsker, D. \& Rasmussen, P. (Eds.), (2021) IOC World Bird List. Ver. 11.1. Available from: https://doi.org/10.14344/ IOC.ML.11.1 (accessed 15 July 2021)

Goldová, M., Paluš, V., Letková, V., Kočišová, A., Čurlík, J. \& Mojžišová, J. (2006) Parasitoses in pheasants (Phasianus colchicus) in confined systems. Veterinarski Arhiv, 76 (Supplement), S83-S89.

Grossi, A.A., Sharanowski, B.J. \& Galloway, T.D. (2014) Anatoecus species (Phthiraptera: Philopteridae) from Anseriformes in North America and taxonomic status of Anatoecus dentatus and Anatoecus icterodes. The Canadian Entomologist, 146, 598-608. https://doi.org/10.4039/tce.2014.12

Grube, A.W. (1851) Klasse Insecta—Parasitae. Fam. Mallophaga. In: Middendorff, A.T. von (Ed.), Reise in den äussersten Norden und Osten Sibiriens während der Jahre 1843-1844. Zoologie 2. St Petersburg, pp. 467-497 \& 516. [in German]

Gurlt, E.F. (1843) Über die auf den Haus-Säugetieren und Haus-Vögeln lebenden Schmarotzer-Insekten und Arachniden. Magazin für die gesammte Thierheilkunde, 9, 1-24. [in German]

Gustafsson, D.R. \& Bush, S.E. (2017) Morphological revision of the hyperdiverse Brueelia-complex (Insecta: Phthiraptera: Ischnocera: Philopteridae) with new taxa, checklists and generic key. Zootaxa, 4313 (1), 1-443. https://doi.org/10.11646/zootaxa.4313.1.1

Gustafsson, D.R., Ošlejšková, L., Najer, T., Sychra, O. \& Zou, F. (2019) Redescriptions of thirteen species of chewing lice in the Brueelia-complex (Phthiraptera, Ischnocera, Philopteridae), with one new synonymy and a neotype designation for Nirmus lais Giebel, 1874. Deutsche Entomologische Zeitschrift, 66 (1), 17-39.

Gustafsson, D.R. \& Zou, F. (2020) Gallancyra gen. nov. (Phthiraptera: Ischnocera), with an overview of the geographical distribution of chewing lice parasitizing chicken. European Journal of Taxonomy, 685, 1-36.

Haeckel, E. (1896) Systematische Phylogenie. 2. Theil. Systematische Phylogenie der wirbellosen Thiere (Invertebrata). Verlag von Georg Reimer, Berlin, 720 pp. [in German] 
Haan, M.W. de (1829) Explication des Planches. In: Lyonet, P. (Ed.), Anatomie de différentes espèces d'insectes. Mémoires du Muséum d'Histoire Naturelle, Paris, 18, pp. 304-312. [in French]

Harrison, L. (1916) The genera and species of Mallophaga. Parasitology, 9 (1), 1-156.

Hopkins, G.H.E. (1941) Stray notes on Mallophaga.--III. 11. The identity of two species of Trichodectes, s. 1. Annals and Magazine of Natural History, Series 11, 7, 36-40

Hopkins, G.H.E. (1947) Notes on Mallophagan nomenclature II. The Entomologist, 80 (1007), 73-79.

Hopkins, G.H.E. (1949) Stray notes on Mallophaga-IX. 56. The various identities of Docophorus auratus. Annals and Magazine of Natural History, Series 12, 2, 29-32.

Hudec, K. (Ed.), (1983) Ptáci 3-I \& II, Fauna ČSSR, svazky 23, 24. [Birds 3-I \& II. Fauna CSSR. Vols. 23 \& 24]. Academia, Praha, 1236 pp. [in Czech]

Hudec, K. \& Černý, W. (Eds.), (1972) Ptáci 1, Fauna ČSSR, svazek 19. [Birds 1. Fauna CSSR. Vol. 19]. Academia, Praha, 536 pp. [in Czech]

Hudec, K. \& Černý, W. (Eds.), (1977) Ptáci 2, Fauna ČSSR, svazek 21. [Birds 2. Fauna CSSR. Vol. 21]. Academia, Praha, 896 pp. [in Czech]

Ilieva, M. (2009) Checklist of the chewing lice (Insecta: Phthiraptera) from wild birds in Bulgaria. Zootaxa, 2138 (1), 1-66. https://doi.org/10.11646/zootaxa.2138.1.1

Janiga M. (2018) Different coevolutionary breeding strategies of Ischnoceran lice on Prunella collaris and P. modularis in high mountains. Polish Journal of Ecology, 66 (2), 182-193.

Janiga M. (2019) Adaptive plasticity in insect parasites - Philopterus lice and their accentor passerine hosts. Polish Journal of Ecology, 66 (4), 395-406.

Janiga, M. \& Kubašková, L. (2000) The biology of the alpine accentor Prunella collaris. III. The coevolution of alpine accentors and lice (Phthiraptera). Oecologia Montana, 9 (1-2), 24-28.

Janiga, M. \& Mičková, A. (2004) The biology of the alpine accentor Prunella collaris. V. The sex ratio and transmission of lice Philopterus emiliae. Oecologia Montana, 13, 17-22.

Johnston, T.H. \& Harrison, L. (1912) A list of Mallophaga found on introduced and domesticated animals in Australia. Proceedings of the Royal Society of Queensland, 24, 17-22.

Kéler, S. von (1936) Über einige Mallophagen aus Rossitten. Arbeiten in morphologische und taxonomische Entomologie von Berlin-Dahlem, 3, 256-264. [in German]

Kéler, S. von (1942) Ein Beitrag zur Kenntnis der Mallophagen. Arbeiten über Morphologische und Taxonomische Entomologie aus Berlin-Dahlem, 9 (2), 69-85. [in German]

Kellogg, V.L. (1896a) New Mallophaga, I,-with special reference to a collection made from maritime birds of the Bay of Monterey, California. Proceedings of the California Academy of Sciences, Series 2, 6, 31-168, 14.

Kellogg, V.L. (1896b) New Mallophaga, II,--from land birds; together with an account of the mallophagous mouth-parts. Proceedings of the California Academy of Sciences, Series 2, 6, 431-548.

Kellogg, V.L. (1899) New Mallophaga III. Mallophaga from birds of Panama, Baja California and Alaska. Occasional Papers of the California Academy of Sciences, 6, 3-52.

Kleven, O., Brøseth, H., Jonassen, K. \& Pedersen, H.C. (2020) Backcrossing of a capercaillie $\times$ black grouse hybrid male in the wild revealed with molecular markers. European Journal of Wildlife Research, 66 (35), 1-4. https://doi.org/10.1007/s10344-020-01377-y

Kose, M. \& Møller, A.P. (1999) Sexual selection, feather breakage and parasites: The importance of white spots on the tail of the barn swallow (Hirundo rustica). Behavioral Ecology and Sociobiology, 45 (6), 430-436. https://doi.org/10.1007/s002650050581

Krištofík, J. (1999) Brueelia balati sp. n. (Phthiraptera, Ischnocera), an ectoparasite of penduline tit (Remiz pendulinus pendulinus). Biologia Bratislava, 54 (2), 139-142.

Krištofík, J. (2000) Synonymical notes to the Menacanthus species (Phthiraptera, Menoponidae) living on Passeriformes (Aves). Acta Parasitologica, 45 (1), 57-58.

Krištofík, J., Manas, P. \& Sustek, K. (1996) Ectoparasites of bee eaters (Merops apiaster) and arthropods in its nests. Biologia Bratislava, 51, 557-570.

Krištofík J. \& Danko Š. (Eds.), (2012) Cicavce Slovenska: rozšǐrení, bionómia a ochrana. [Mammals of Slovakia: distribution, bionomy and protection]. VEDA-Vydavatelstvo Slovenskej Akadémie vied, Bratislava, 712 pp. [in Slovak]

Kvetko, R. \& FK SOS/BirdLife Slovensko (2017) 17. Správa Faunistickej komisie Slovenskej ornitologickej spoločnosti/ BirdLife Slovensko. [The 17th report of the Rarities Committee of the Slovak Ornithological Society/BirdLife Slovakia]. Tichodroma, 29, 42-48. [in Slovak]

Liebers, D., Helbig, A.J. \& de Knijff, P. (2001) Genetic differentiation and phylogeography of gulls in the Larus cachinnansfuscus group (Aves: Charadriiformes). Molecular Ecology, 10, 2447-2462.

Linnaeus, C. von (1758) Systema Naturae per regna tria naturae, secundum classes, ordines, genera, species, cum characteribus, differentiis, synonymis, locis. Tenth Edition. Volume 1. Laurentii Salvii, Holmiae, iv + 824 pp. [in Latin]

Linnaeus, C. (1761) Pediculus. In: Linnaeus, C., Fauna Suecica, sistens Animalia sveciae regni: Mammalia, Aves, Amphibia, Pisces, Insecta, Vermes. Laurentii Salvii, Stockholmiae, pp. 475-479. [in Latin]

Literák, I., Sychra, O., Resendes, R. \& Rodrigues, P. (2015) Chewing lice in Azorean blackcaps (Sylvia atricapilla): A contribution to parasite island syndromes. Journal of Parasitology, 101, 252-254. 
https://doi.org/10.1645/14-601.1

Lukáš, J., Cyprich, D. \& Krumpál, M. (1991) Trichodectes melis (J.C. Fabricius, 1805) (Mallophaga, Trichodectidae) nový druh pre územie Slovenska. [Trichodectes melis (J.C. Fabricius, 1805) (Mallophaga, Trichodectidae), a new species for the fauna of Slovakia]. Biologia Bratislava, 46, 967-968. [in Slovak]

Lukáš, J., Cyprich, D. \& Krumpál, M. (1992) Príspevok kvýskytu srstiarky jazvečej (Trichodectes melis J.C. Fabricius, 1805) na Slovensku. [The contribution to the occurrence of Trichodectes melis (J.C. Fabricius, 1805) in Slovakia]. Správy Slovenskej entomologickej spoločnosti pri SAV, 1992, 8-11. [in Slovak]

Lunkaschu, M.I. (1970) Two new species of Mallophaga of the genus Brueelia Kéler from passerines of Moldavia. Izvesti Akademy Nauk Moldavsko SSR, Series Biology, 1, 53-57. [in Russian]

Máca, J. (1991) Mallophaga parasitizing mammals in Czechoslovakia. Acta Societatis Zoologicae Bohemicae, 55, 1-11.

Martín-Mateo, M.P. (1981) Una nueva especie de Colpocephalum Nitzsch (Mallophaga), parásita de águila imperial. Eos, Madrid, 55-56 (1979-1980), 107-113. [in Spanish]

Martinů, J., Sychra, O., Literák, I., Čapek, M., Gustafsson, D.R. \& Štefka, J. (2015) Host generalists and specialists emerging side by side: an analysis of evolutionary patterns in the cosmopolitan chewing louse genus Menacanthus. International Journal for Parasitology, 45, 63-73. https://doi.org/10.1016/j.ijpara.2014.09.001

Macháček, P. (1977) Ektoparaziti vrabce domaciho Passer domesticus (L.) a vrabce polniho Passer montanus (L.). [Ectoparasites of house sparrow Passer domesticus (L.) and tree sparrow Passer montanus (L.)]. Scripta Facultatis Scientiarum UJEP Brunensis. Biologia, 2, 71-86. [in Czech]

Merisuo, A.K. (1945) Notulae mallophagologicae. II Die Craspedorrhynchus-Arten Finnlands, Federlinge unserer Accipitres. Annales Entomologici Fennici, 11, 101-112. [in German]

Mey, E. (1977) Über den Mallophagen-Befall bei einigen Rohrsängern und Meisen. Der Falke, Monatsschrift für Ornithologie und Vogelschutz, 24, 402-404. [in German]

Mey, E. (1983) Docophorulus fedorenkoae sp. n. (Mallophaga). Vestnik Zoologii, Kiev, 1983 (2), 31-35. [in Russian]

Mey, E. (1988) Zur taxonomie der auf meisen (Paridae) parasitierenden Docophorulus — arten (Insecta, Phthiraptera, Philopteridae). Rudolstädter Naturhistorische Schriften, 1, 71-77. [in German]

Mey, E. (2003) Verzeichnis der Tierläuse (Phthiraptera) Deutschlands. In: Klausnitzer, B. (Ed.), Entomofauna Germanica 6. Vol. 8. Entomologische Nachrichten und Berichte, Beiheft Dresden, pp. 72-129. [in German]

Mey, E. (2004) Zur Taxonomie, Verbreitung und parasitophyletische Evidenz des Philopterus-Komplexes (Insecta, Phthiraptera, Ischnocera). Ornithologischer Anzeiger, 43, 149-203. [in German]

Mey, E. (2021) Phthiraptera. In: de Jong, Y. (Ed.), Fauna Europaea. Fauna Europaea Consortium. Checklist dataset. Available from: https://www.gbif.org/ (accessed 21 July 2021) [in German] https://doi.org/10.15468/ymk1bx

Mjöberg, E. (1910) Studien über Mallophagen und Anopluren. Arkiv för Zoologi, 6 (13), 1-296. [in German]

Najer, T., Papoušek, I., Adam, C., Trnka, A., Quach, V.T., Nguyen, C.N., Figura, R., Literák, I. \& Sychra, O. (2020) New records of Philopterus (Ischnocera: Philopteridae) from Acrocephalidae and Locustellidae, with description of one new species from Regulidae. European Journal of Taxonomy, 632, 1-37. https://doi.org/10.5852/ejt.2020.632

Naz, S., Rizvi, S.A. \& Sychra, O. (2010) The high rate of infestation of chewing lice (Phthiraptera) in rock pigeons (Columba livia Gmelin, 1789) in Pakistan. Tropical Zoology, 23, 21-28.

Neumann, L.-G. (1890) Contribution a l'etude des Ricinidae parasites des oiseaux de la famille des Psittacidae. Bulletin de la Société d'Historie Naturelle, Toulouse, 24, 55-69. [in French]

Nitzsch, C.L. (1818) Die Familien und Gattungen der Theierinsekten (Insecta epizoica); als ein Prodromus einer Naturgeschichte derselben. E.F. Germar's Magazin der Entomologie, 3, 261-318. [in German]

Nitzsch, C.L. (1866) Die Federlinge der Sing-, Schrei-, Kletter- und Taubenvögel. Zeitschrift für die gesammten Naturwissenschaften, Halle, 27 (2), 115-122. [in German]

Olfers, I.F.J.M. von (1816) De vegetativis et animatis corporibus in corporibus animatis reperiundis commentarius. Taberna Libraria Maureriana, Berolini, vi + 113 pp. [in Latin]

Osborn, H. (1902) Mallophagan records and descriptions. IV. New species and records in Colpocephalum and Physostomum. Ohio Naturalist, 2 (4), 201-204.

Ošlejšková, L., Kounková, Š., Gustafsson, R.D., Resendes, R., Rodriguez, P., Literák, I. \& Sychra, O. (2020) Insect ectoparasites from wild passerine birds in the Azores Islands. Parasite, 27 (64), 1-16. https://doi.org/10.1051/parasite/2020063

Pérez, J.M., Ruiz Martínez, I. \& Cooper, J.E. (1996) Occurrence of chewing lice on Spanish raptors. Ardeola, 43, $129-138$.

Piaget, E. (1880) Les Pédiculines. Essai Monographique. Vols. 1-2. E.J. Brill, Leide, xxxix + 71456 pp. [in French]

Piaget, E. (1890) Quelques pédiculines nouvelles. Tijdschrift voor Entomologie, 33, 223-259. [in French]

Price, R.D. (1975) The Menacanthus eurysternus complex (Mallophaga: Menoponidae) of the Passeriformes and Piciformes (Aves). Annals of the Entomological Society of America, 68 (4), 617-622.

Price, R.D. (1977) The Menacanthus (Mallophaga: Menoponidae) of the Passeriformes (Aves). Journal of Medical Entomology, 14 (2), 207-220.

Price, R.D., Hellenthal, R.A., Palma, R.L., Johnson, K.P. \& Clayton, D.H. (2003) The chewing lice: world checklist and biologi- 
cal overview. Illinois Natural History Survey Special Publication 24. Illinois Natural History Survey, Champaign, Illinois, $\mathrm{x}+501 \mathrm{pp}$.

Rak, H., Anwar, M. \& Niak, A. (1975) The species of mallophaga in wild birds in Iran. Bulletin de la Société de Pathologie Exotique et de ses Filiales, 68 (6), 588-591.

Rékási, J., Kiss, J.B. \& Sándor, A.D. (2017) Chewing lice (Phthiraptera: Amblycera, Ischnocera) recorded from birds in the Danube Delta Biosphere Reserve: a literature review with new data. Aquila, 124, 7-33.

Rózsa, L. \& Vas, Z. (2015) Co-extinct and critically co-endangered species of parasitic lice, and conservation-induced extinction: should lice be reintroduced to their hosts? Oryx, 49, 107-110. https://doi.org/10.1017/S0030605313000628

Rudow, F. (1866) Charakteristik neuer Federlinge. Zeitschrift für die Gesammten Naturwissenschaften, Halle, Neue Folge 2, 27 (6), 465-477 [in German]

Rudow, F. (1869a) Neue Mallophagen. Zeitschrift für die gesammten Naturwissenschaften, Halle, Neue Folge 2, 34 (11/12), 387-407. [in German]

Rudow, F. (1869b) Beitrag zur Kenntniss der Mallophagen oder Pelzfresser. Neue exotische Arten der Familie Philopterus. Inaugural—Dissertation, Universität zu Leipzig. Druck von Wilh, Plötz. Halle, 47 pp. [in German]

Rudow, F. (1870) Beobachtungen über die Lebensweise und den Bau der Mallophagen oder Pelzfresser sowie Beschreibung neuer Arten. Zeitschrift für die gesammten Naturwissenschaften, Halle, Neue Folge 1, 35 (6), 449-487. [in German]

Sangster, G. \& Oreel, G.J. (1996) Progress in taxonomy of taiga and tundra bean geese. Dutch Birding, 18, $310-316$.

Schrank, F. von P. (1776) Beyträge zur Naturgeschichte. Veith, Leipzig, 140 pp. [in German]

Schrank, F. von P. (1803) Thierlaus. Fauna Boica, Landshut, 3 (1), 186-194. [in German]

Scopoli, J.A. (1763) Entomologia Carniolica exhibens insecta Carnioliae indigena et distributa in ordines, genera, species, varietates, methodo Linnaeana. Trattner, Wien, xxxvi + 421 pp. [in Latin]

Scopoli, J.A. (1772) Observationes Zoologicae. Pediculus. In: Scopoli, J.A., Annus V. Historico-Naturalis, G. Hilscheri, Lipsiae, pp. 124-125. [in Latin]

SOS/BirdLife Slovakia (2020) List of birds of Slovakia by the Faunistic Commission of the Slovak Ornithological Society/ BirdLife Slovakia. Available from: https://www.vtaky.sk/stranka/45-Zoznam-druhov-SR.html (accessed 15 July 2021)

Šrank, V. (2010) 10. Správa Slovenskej faunistickej komisie Slovenskej ornitologickej spoločnosti/BirdLife Slovensko. [The 10th Report of the Slovak Rarities Committee of the Slovak Ornithological Society/BirdLife Slovakia]. Tichodroma, 22, 131-134. [in Slovak]

Štefan, P. (1977) Trichodectes melis (J.C. Fabricius, 1805), (Mallophaga, Trichodectidae), nový druh všenky z území Slovenska. [Trichodectes melis (J.C. Fabricius, 1805), (Mallophaga, Trichodectidae), a new species chewing louse for the fauna of Slovakia]. Biologia Bratislava, 32, 367-369. [in Slovak]

Straka, V. (1982) Forézia švol (Mallophaga) dvojkrídlovcami. [Phoresis of chewing lice (Mallophaga) on dipterans]. Kmentianum, Martin, 3, 257-259. [in Slovak]

Straka, V. (1987) Prehl’ad švol (Mallophaga) v zbierkach Turčianskeho múzea Andreja Kmet’a v Martine. [A survey of Mallophaga in the collections of Andrej Kmet Turcianske Museum in Martin]. Kmetianum, Martin, 8, 321-331. [in Slovak]

Sychra, O., Jensen, J.-K., Brooke, M. de L., Trnka, A., Procházka, P. \& Literák, I. (2008) The identity of Menacanthus eisenachensis Balát (Insecta, Phthiraptera, Amblycera, Menoponidae) from the Reed Warbler (Passeriformes, Sylviidae). Acta Parasitologica, 53 (4), 404-406. https://doi.org/10.2478/s11686-008-0060-5

Sychra, O., Kolencik, S., Papousek, I., Bilbija, B. \& Literak, I. (2021) Myrsidea quadrifasciata (Phthiraptera: Amblycera)—a unique host generalist among highly host-specific chewing lice. Arthropod Systematics \& Phylogeny, 79, 379-400. https://doi.org/10.3897/asp.79.e6397

Sychra, O., Kounek, F., Papoušek, I., Čapek, M., Cárdenas-Callirgos, J.M., Franco, S. \& Literák, I. (2014) Chewing lice (Phthiraptera: Amblycera et Ischnocera) from wrens (Passeriformes: Troglodytidae), with description of a new species of Myrsidea. Acta Entomologica Musei Nationalis Pragae, 54 (1), 1-27.

Szczykutowicz, A., Adamski, Z., Hromada, M. \& Tryjanowski, P. (2006) Patterns in the distribution of avian lice (Phthiraptera: Amblycera, Ischnocera) living on the great grey shrike Lanius excubitor. Parasitology Research, 14, 1-4. https://doi.org/10.1007/s00436-005-0108-5

Tandan, B.K. (1951) Mallophagan parasites from Indian birds. Part I. New species belonging to the genera Aquanirmus, Quadraceps and Rallicola (Ischnocera: Philopteridae). Annals and Magazine of Natural History, Series 12, 48, 802-813.

Tandan, B.K. (1955) Mallophagan parasites from Indian birds. Part IV. Species belonging to the genera Philopterus, Capraiella and Pectinopygus (superfamily Ischnocera). Annals and Magazine of Natural History, Series 12, 8, 417-433.

Taschenberg, O. (1882) Die mallophagen mit besonderer Berücksichtigung der von Dr. Meyer gesammelten Arten systematisch bearbeitet. Nova Acta der Kaiserlich Leopoldinisch-Carolinisch Deutschen Akademie der Naturforscher, 44 (1), 1-244. [in German]

Tompkins, D.M., Jones, T. \& Clayton, D.H. (1996) Effect of vertically transmitted ectoparasites on the reproductive success of swifts (Apus apus). Functional Ecology, 10, 733-740.

Uchida, S. (1915) Bird-infesting Mallophaga of Japan. (Genus Physostomum). Annotationes zoologicae Japonenses, 9 (1), 67-72.

Uchida, S. (1916) Bird-infesting Mallophaga of Japan II. (Genera Goniodes and Goniocotes). Annotationes zoologicae Japon- 
enses, 9 (2), 81-88.

Vas, Z., Rékási, J. \& Rózsa, L. (2012a) A checklist of lice of Hungary (Insecta: Phthiraptera). Annales historico-naturales Musei nationalis hungarici, 104, 5-109.

Vas, Z., Privigyei, C., Prohaszka, V.J., Csorgo T. \& Rózsa, L. (2012b) New species and host association records for the Hungarian avian louse fauna (Insecta: Phthiraptera). Ornis Hungarica, 20 (1), 44-49. https://doi.org/10.2478/orhu-2013-0004

Złotorzycka, J. (1964a) Mallophaga parasitizing Passeriformes and Pici II. Brueeliinae. Acta Parasitologica Polonica, 12 (24), 239-282.

Złotorzycka, J. (1964b) Mallophaga parasitizing Passeriformes and Pici III. Philopterinae. Acta Parasitologica Polonica, 12 (37), 401-430.

Złotorzycka, J. (1965) Mallophaga parasitizing Passeriformes and Pici IV. Menacanthinae, Ricinidae, Degeeriellinae. Acta Parasitologica Polonica, 13 (6), 41-70.

Złotorzycka, J. (1966) Systematische Bemerkungen über die Gattung Reticulipeurus Kéler mit Beschreibung von R. tetraonis minor ssp. n. (Mallophaga, Lipeuridae). Polskie Pismo Entomologiczne, 36 (9), 111-115. [in German]

Złotorzycka, J. (1968) Systematische Studien an den mitteleuropäischen Arten der Gattung Austromenopon Bedf. (Mallophaga, Austromenoponinae). Polskie Pismo Entomologiczne, 38 (2), 301-340. [in German]

Złotorzycka, J. (1972a) Wszoly -Mallophaga, zeszyt 1, Czesc ogolna oraz nadrodziny Gyropoidea i Laemobothrioidea. [Chewing lice-Mallophaga 1, General introduction and Superfamilies Gyropoidea and Laemobothrioidea]. Klucze do Oznaczania Owadów Polski 15. Panstwowe Wydawnictwo Naukowe, Warszawa \& Wroclaw, 57 pp. [in Polish]

Złotorzycka, J. (1972b) Wszoly -Mallophaga, zeszyt 3, Nadrodziny Goniodoidea i Trichodectoidea. [Chewing lice—Mallophaga 1, Superfamilies Goniodoidea i Trichodectoidea]. Klucze do Oznaczania Owadów Polski 15. Panstwowe Wydawnictwo Naukowe, Warszawa \& Wroclaw, 48 pp. [in Polish]

Złotorzycka, J. (1976) Wszoly-Mallophaga, zeszyt 2, Nadrodzina Menoponoidea. [Chewing lice-Mallophaga 2, Superfamily Menoponoidea]. Klucze do Oznaczania Owadów Polski 15. Panstwowe Wydawnictwo Naukowe, Warszawa \& Wroclaw, 190 pp. [in Polish]

Złotorzycka, J. (1977) Wszoly_Mallophaga, zeszyt 4, Nadrodzina Philopteroidea, rodzina Philopteridae. [Chewing lice—Mallophaga 4, Superfamily Philopteroidea, family Philopteridae]. Klucze do Oznaczania Owadów Polski 15, Panstwowe Wydawnictwo Naukowe, Warszawa \& Wroclaw, 124 pp. [in Polish]

Złotorzycka, J. (1978) Wszoly-Mallophaga, zeszyt 5, Nadrodzina Philopteroidea, rodzina Rallicolidae. [Chewing lice-Mallophaga 5, Superfamily Philopteroidea, family Rallicolidae]. Klucze do Oznaczania Owadów Polski 15. Panstwowe Wydawnictwo Naukowe, Warszawa \& Wroclaw, 85 pp. [in Polish]

Złotorzycka, J. (1980) Wszoly_Mallophaga, zeszyt 6, Nadrodzina Philopteroidea, rodziny: Meinertzhageniellidae, Lipeuridae, Degeeriellidae, Pseudonirmidae, Giebeliidae, Esthiopteridae i Acidoproctidae. [Chewing lice-Mallophaga 6, Superfamily Philopteroidea, families Meinertzhageniellidae, Lipeuridae, Degeeriellidae, Pseudonirmidae, Giebeliidae, Esthiopteridae i Acidoproctidae]. Klucze do Oznaczania Owadów Polski 15. Panstwowe Wydawnictwo Naukowe, Warszawa \& Wroclaw, 202 pp. [in Polish] 